\title{
ARL Annual Salary Survey 2007-2008
}

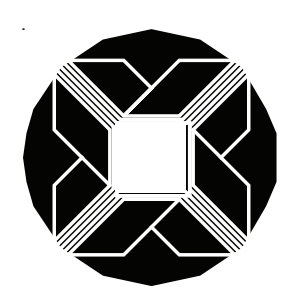

Compiled and Edited by

Martha Kyrillidou

MARK YOUNG

JASON BARBER

Association of Research Libraries

Washington, D.C.

2008 
ARL Annual Salary Survey is published by

Association of Research Libraries

21 Dupont Circle, NW, Suite 800

Washington, D.C. 20036

Telephone: (202) 296-2296

FAX: (202) 872-0884

email: pubs@arl.org

Custom reports based on the Salary Survey data are also available. Contact the ARL Statistics and Measurement Program Officer for further information.

The quantitative rank order tables presented in this publication are not indicative of performance and outcomes and should not be used as measures of library quality. In comparing any individual library to ARL medians or to other ARL members, one must be careful to make such comparisons within the context of differing institutional and local goals and characteristics.

Visit the ARL Statistics and Measurement Program online at http:/ / www.arl.org/stats/.

\section{EAN 978-159407-809-5 \\ ISBN 1-59407-809-2}

(C) 2008

The compilation is copyrighted by the Association of Research Libraries. Blanket permission is granted to reproduce and distribute copies of this work for nonprofit, educational, or library purposes, provided that the author, source, and copyright notice are included on each copy. This permission is in addition to rights of reproduction granted under Sections 107, 108, and other provisions of the U.S. Copyright Act.

The paper used in this publication meets the minimum requirements of the American National Standard for Information Science and National Information Standards Organization standard-Permanence of Paper for Publications and Documents in Libraries and Archives, ANSI/NISO Z39.48-1992(R1997). 


\section{TABLE OF CONTENTS}

Page

SALARY SURVEY TRENDS 2007-2008 . .7

\section{SALARY LEVELS FOR STAFF IN ARL LIBRARIES}

Table 1: Distribution by Salary Level .18

Table 2: $\quad$ Salary Trends in ARL University Libraries. 19

Table 3: $\quad$ Salary Trends in U.S. ARL University Libraries. 20

Table 4: $\quad$ Salary Trends in Canadian ARL University Libraries .21

\section{ARL NONUNIVERSITY LIBRARIES}

Table 5: Median and Beginning Professional Salaries in ARL Nonuniversity Libraries .24

Table 6: $\quad$ Salary Trends in ARL Nonuniversity Libraries .25

\section{ARL UNIVERSITY LIBRARIES}

Table 7: $\quad$ Filled Positions; Average, Median, Beginning Professional Salaries; and Average Years of Experience in ARL University Libraries, FY 2007-2008 .28

Table 8: $\quad$ Beginning Professional Salaries in ARL University Libraries; Rank Order Table, FY 2006-2007. .30

Table 9: $\quad$ Beginning Professional Salaries in ARL University Libraries; Rank Order Table, FY 2007-2008. .31

Table 10: $\quad$ Median Professional Salaries in ARL University Libraries; Rank Order Table, FY 2006-2007. .32

Table 11: Median Professional Salaries in ARL University Libraries; Rank Order Table, FY 2007-2008. .33

Table 12: $\quad$ Average Professional Salaries in ARL University Libraries; Rank Order Table, FY 2006-2007 34

Table 13: $\quad$ Average Professional Salaries in ARL University Libraries; Rank Order Table, FY 2007-2008 .35

Table 14: Average, Median, and Beginning Professional Salaries in ARL University Libraries; Summary of Rankings, FYs 2004-2005 to 2007-2008 .36

Table 15: $\quad$ Distribution of Professional Staff in ARL University Libraries by Salary and Position, FY 2007-2008 
Table 16: Distribution of Professional Staff in ARL University Libraries

by Salary, Sex, and Position, FY 2007-2008

Table 17: $\quad$ Number and Average Salaries of ARL University Librarians

by Position and Sex, FY 2007-2008

Table 18: $\quad$ Number and Average Years of Experience of ARL University Librarians

by Position and Sex, FY 2007-2008

Table 19: $\quad$ Number and Average Salaries of ARL University Librarians

by Years of Experience and Sex, FY 2007-2008

Table 20: $\quad$ Average Salaries of ARL University Librarians

by Position and Years of Experience, FY 2007-2008

Table 21: $\quad$ Number and Average Salaries of ARL University Librarians

by Position and Type of Institution, FY 2007-2008.

Table 22: $\quad$ Years of Experience of ARL University Librarians

by Position and Type of Institution, FY 2007-2008.

Table 23: $\quad$ Number and Average Salaries of ARL University Librarians

by Position and Size of Professional Staff, FY 2007-2008.

Table 24: $\quad$ Years of Experience of ARL University Librarians

by Position and Size of Professional Staff, FY 2007-2008.

Table 25: $\quad$ Average Salaries of ARL University Librarians

by Position and Geographic Region, FY 2007-2008

ARL University Libraries by Geographic Region .51

\section{U.S. ARL UNIVERSITY LIBRARIES}

Table 26: $\quad$ Average Salaries of U.S. ARL University Librarians

by Position and Years of Experience, FY 2007-2008

Table 27: $\quad$ Number and Average Salaries of Minority U.S. ARL University Librarians

by Position and Sex, FY 2007-2008 .55

Table 28: $\quad$ Number and Average Years of Experience of Minority U.S. ARL University Librarians by Position and Sex, FY 2007-2008.

Table 29: $\quad$ Number and Average Salaries of U.S. ARL University Librarians

by Years of Experience and Sex, FY 2007-2008

Table 30: $\quad$ Number and Average Salaries of Minority U.S. ARL University Librarians

by Years of Experience and Sex, FY 2007-2008 


\section{CANADIAN ARL UNIVERSITY LIBRARIES}

Table 31: $\quad$ Filled Positions; Average, Median, and Beginning Professional Salaries; and Average Years of Professional Experience in Canadian ARL University Libraries, FY 2007-2008

Table 32: $\quad$ Number and Average Salaries of Canadian ARL University

Librarians by Position and Sex, FY 2007-2008.

Table 33: $\quad$ Number and Average Years of Experience of Canadian ARL

University Librarians by Position and Sex, FY 2007-2008

Table 34: $\quad$ Number and Average Salaries of Canadian ARL University Librarians

by Years of Experience and Sex, FY 2007-2008

\section{ARL UNIVERSITY MEDICAL LIBRARIES}

Table 35: $\quad$ Filled Positions; Average, Median, Beginning Professional Salaries; and Average

Years of Professional Experience in ARL University Medical Libraries, FY 2007-2008 .66

Table 36: $\quad$ Beginning Professional Salaries in ARL University Medical Libraries;

Rank Order Table, FY 2007-2008.

Table 37: $\quad$ Median Professional Salaries in ARL University Medical Libraries;

Rank Order Table, FY 2007-2008.

Table 38: $\quad$ Average Professional Salaries in ARL University Medical Libraries;

Rank Order Table, FY 2007-2008.

Table 39: $\quad$ Number and Average Salaries of ARL University Medical Librarians

by Position and Sex, FY 2007-2008 .71

Table 40: $\quad$ Number and Average Years of Experience of ARL University Medical Librarians by Position and Sex, FY 2007-2008 .72

Table 41: $\quad$ Number and Average Salaries of ARL University Medical Librarians by Years of Experience and Sex, FY 2007-2008

\section{ARL UNIVERSITY LAW LIBRARIES}

Table 42: $\quad$ Filled Positions; Average, Median, Beginning Professional Salaries; and Average

Years of Experience in ARL University Law Libraries, FY 2007-2008

Table 43: $\quad$ Beginning Professional Salaries in ARL University Law Libraries;

Rank Order Table, FY 2007-2008.

Table 44: $\quad$ Median Professional Salaries in ARL University Law Libraries;

Rank Order Table, FY 2007-2008.

Table 45: $\quad$ Average Professional Salaries in ARL University Law Libraries;

Rank Order Table, FY 2007-2008. 
Table 46: $\quad$ Number and Average Salaries of ARL University Law Librarians by Position and Sex, FY 2007-2008

Table 47: Number and Average Years of Experience of ARL University Law Librarians by Position and Sex, FY 2007-2008

Table 48: $\quad$ Number and Average Salaries of ARL University Law Librarians

by Years of Experience and Sex, FY 2007-2008

\section{ARL ANNUAL SALARY SURVEY 2007-2008}

University Library Questionnaire and Instructions .85

Nonuniversity Library Questionnaire and Instructions .97

FOOTNOTES .101

APPENDIX A: ARL MEMBER LIBRARIES .110

APPENDIX B: TABLE NUMBERING CHANGES FOR 1998-99 to 1999-2000 


\section{SALARY SURVEY TRENDS 2007-2008}

The ARL Annual Salary Survey 2007-2008 reports salary data for all professional staff working in ARL libraries. The Association of Research Libraries (ARL) represents the interests of libraries that serve major North American research institutions. The Association operates as a forum for the exchange of ideas and as an agent for collective action to influence forces affecting the ability of these libraries to meet the future needs of scholarship. The ARL Statistics and Measurement program, which produces the Salary Survey, is organized around collecting, analyzing, and distributing quantifiable information describing the characteristics of research libraries. The ARL Annual Salary Survey is the most comprehensive and thorough guide to current salaries in large U.S. and Canadian academic and research libraries, and is a valuable management and research tool.

Data for 9,983 professional staff members were reported this year for the 113 ARL university libraries, including their law and medical libraries (937 staff members reported by 73 medical libraries and 732 staff members reported by 75 law libraries). For the 10 nonuniversity ARL members, data were reported for 3,797 professional staff members.

The tables are organized in seven major sections. The first section includes Tables 1 through 4 , which report salary figures for all professionals working in ARL member libraries, including law and medical library data. The second section includes salary information for the 10 nonuniversity research libraries of ARL. The third section, entitled "ARL University Libraries," reports data in Tables 7 through 25 for the "general" library system of the university ARL members, combining U.S. and Canadian data but excluding law and medical data. The fourth section, composed of Tables 26 through 30, reports data on U.S. ARL university library members excluding law and medical data; the fifth section, Tables 31-34, reports data on Canadian ARL university libraries excluding law and medical data. The sixth section (Tables 35-41) and the seventh section (Tables 42-48) report on medical and law libraries, respectively, combining U.S. and Canadian data.

The university population is generally treated in three distinct groups: staff in the "general" library system, staff in the university medical libraries, and staff in the university law libraries. Any branch libraries for which data were received, other than law and medical, are included in the "general" category, whether or not those libraries are administratively independent. Footnotes for many institutions provide information on branch inclusion or exclusion.

In all tables where data from U.S. and Canadian institutions are combined, Canadian salaries are converted into U.S. dollar equivalents at the rate of 1.1323 Canadian dollars per U.S. dollar. ${ }^{1}$ Tables 4 and 31 through 34, however, pertain exclusively to staff in Canadian university libraries, so salary data in those tables are expressed in Canadian dollars.

\footnotetext{
1 This is the average monthly noon exchange rate published in the Bank of Canada Review for the period July 2006-June 2007 and is used in converting 2007-2008 figures that are collected as of July 2007.
} 


\section{RACE AND EThNiCITY}

There were 1,280 minority professional staff reported in 99 U.S. ARL university libraries, including law and medical. ${ }^{2}$ Note that the data for minority professionals comes only from the U.S. ARL university libraries following the Equal Employment Opportunity Commission (EEOC) definitions; Canadian law prohibits the identification of Canadians by ethnic category.

Currently, $14.1 \%$ of the professional staff in U.S. ARL university libraries (including law and medical) belongs to one of the four non-Caucasian categories for which ARL keeps records. The number of minorities in managerial or administrative positions in the largest U.S. academic libraries is far lower: 5.2\% are directors (5 out of 97), $6.3 \%$ are associate or assistant directors (26 out of 411 ), and $11.7 \%$ are branch librarians (52 out of 445). Graph 1, below, depicts the overall racial/ethnic distribution of professional staff in U.S. ARL university libraries: Caucasian/Other 85.8\%, Asian/ Pacific Islander 6.3\%, Black 4.8\%, Hispanic 2.7\%, and American Indian/ Alaskan Native $0.3 \%$. According to a 1998 survey by Mary Jo Lynch, data from the American Library Association (ALA) show that the sample of academic libraries surveyed by ALA has a higher representation of Blacks, Asian/ Pacific Islanders, and American Indian/ Alaskan Native than ARL libraries. ${ }^{3}$

\section{Graph 1 \\ Ethnicity/Race of Professional Staff in \\ U.S. ARL University Libraries, 2007-08}

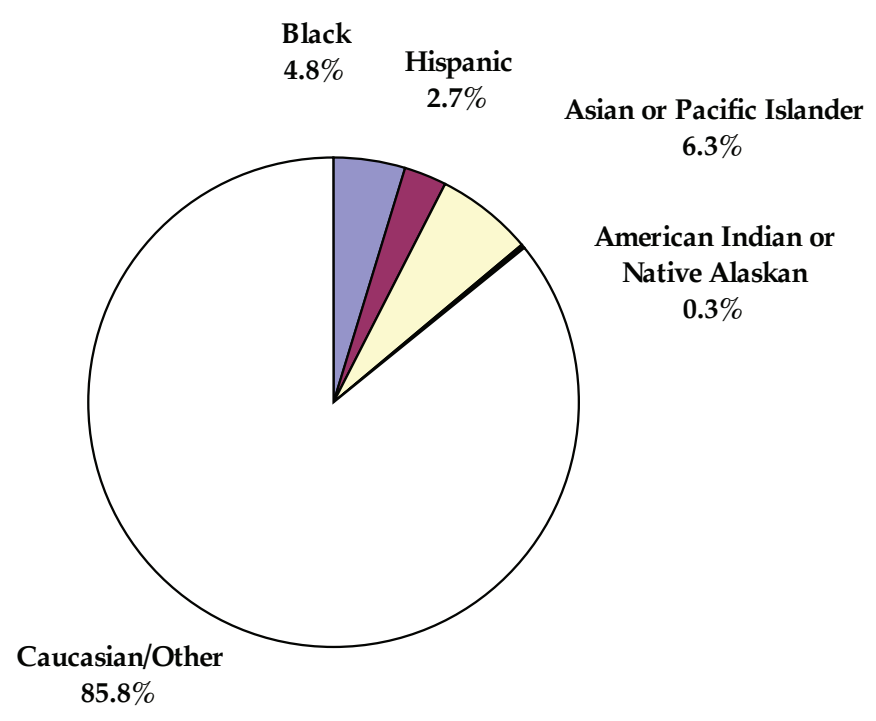

\footnotetext{
${ }^{2}$ Some U.S. institutions offer their librarians the option of not reporting race and ethnicity; others forbid the tracking of racial and ethnic classification altogether. See Footnotes.

3 Mary Jo Lynch, “Librarians' Salaries: Smaller Increases This Year,” American Libraries 29.10 (1998): 66-70. Also available at <http:/ / www.ala.org/alaorg/ors/racethnc.html>.
} 
Minority professional staff in U.S. ARL university libraries continues to be disproportionately distributed across the country. Using Figure 1, we can compare the number of minority staff with other staff, region by region. These patterns of distribution have been relatively stable for the entire history of ARL's data-collection experience. Minorities are underrepresented by more than $30 \%$ in the New England, West North Central, and East South Central regions (see Table 25 for a definition of the regions). Proportionately to other regions, there are more minorities in the South Atlantic, West South Central, and Pacific regions.

FIGURE 1

MINORITY PROFESSIONALS BY REGION (U.S.)

IN ARL UNIVERSITY LIBRARIES, FY 2007-2008

\begin{tabular}{|c|c|c|c|c|c|c|c|c|c|c|c|}
\hline & $\begin{array}{c}\text { New } \\
\text { England }\end{array}$ & $\begin{array}{l}\text { Middle } \\
\text { Atlantic }\end{array}$ & $\begin{array}{l}\text { E North } \\
\text { Central }\end{array}$ & $\begin{array}{c}\text { W } \\
\text { North } \\
\text { Central }\end{array}$ & $\begin{array}{c}\text { South } \\
\text { Atlantic }\end{array}$ & $\begin{array}{c}\text { East } \mathbf{S} \\
\text { Central }\end{array}$ & $\begin{array}{l}\text { West S } \\
\text { Central }\end{array}$ & Mountain & Pacific & TOTAL & $\%$ \\
\hline $\begin{array}{l}\text { Race/Ethnicity } \\
\text { Category }\end{array}$ & & & & & & & & & & & \\
\hline Black & 34 & 63 & 94 & 21 & 136 & 20 & 27 & 9 & 31 & 435 & $34 \%$ \\
\hline Hispanic & 23 & 37 & 29 & 10 & 46 & 5 & 36 & 23 & 39 & 248 & $19 \%$ \\
\hline Asian & 73 & 95 & 76 & 21 & 79 & 10 & 38 & 22 & 156 & 570 & $45 \%$ \\
\hline $\mathbf{A I} / \mathbf{A} \mathbf{N}^{*}$ & 4 & 2 & 4 & 2 & 7 & N/A & 2 & 6 & N/A & 27 & $2 \%$ \\
\hline Minority Total & 134 & 197 & 203 & 54 & 268 & 35 & 103 & 60 & 226 & 1,280 & $100 \%$ \\
\hline $\begin{array}{l}\text { Minority } \\
\text { Percent }\end{array}$ & $10.47 \%$ & $15.39 \%$ & $15.86 \%$ & $4.22 \%$ & $20.94 \%$ & $2.73 \%$ & $8.05 \%$ & $4.69 \%$ & $17.66 \%$ & & \\
\hline $\begin{array}{l}\text { Nonminority } \\
\text { Total }\end{array}$ & 1,165 & 1,250 & 1,378 & 527 & 1,215 & 366 & 557 & 462 & 826 & 7,746 & $100 \%$ \\
\hline $\begin{array}{l}\text { Nonminority } \\
\text { Percent }\end{array}$ & $15.04 \%$ & $16.14 \%$ & $17.79 \%$ & $6.80 \%$ & $15.69 \%$ & $4.73 \%$ & $7.19 \%$ & $5.96 \%$ & $10.66 \%$ & & \\
\hline $\begin{array}{l}\text { Regional } \\
\text { Percent } \\
\text { Total staff }\end{array}$ & $14.39 \%$ & $16.03 \%$ & $17.52 \%$ & $6.44 \%$ & $16.43 \%$ & $4.44 \%$ & $7.31 \%$ & $5.78 \%$ & $11.66 \%$ & & \\
\hline $\begin{array}{l}\text { Proportional } \\
\text { Minority } \\
\text { Representation }\end{array}$ & $-30.39 \%$ & $-4.63 \%$ & $-10.85 \%$ & $-37.99 \%$ & $33.48 \%$ & $-42.13 \%$ & $11.91 \%$ & $-21.41 \%$ & $65.58 \%$ & & \\
\hline
\end{tabular}

* American Indian/Alaskan Native

ARL recognizes the difficulties that the profession has in attracting a diverse workforce and continues to work actively in the development of workplace climates that embrace diversity. The ARL Diversity Program, through its Leadership and Career Development Program and the Initiative to Recruit a Diverse Workforce, emphasizes ARL and its members' commitment to creating a diverse academic and research library community to better meet the new challenges of global competition and changing demographics. 
Further, the Diversity Program focuses on issues surrounding work relationships in libraries while considering the impact of diversity on library services, interactions with library users, and the development of collections, at its homepage, http: / / www.arl.org/diversity / index.html.

Women comprise $69.38 \%$ of the four racial/ ethnic groups that comprise minority staff, as compared to 63.04\% of Caucasian/Other staff in all U.S. ARL university libraries. The overall gender balance in the 113 Canadian and U.S. university libraries (including law and medical) is $35.47 \%$ male and $64.35 \%$ female. See Figure 1, above, and Figure 2, below, for more detail on race/ ethnic and gender distribution.

FIGURE 2

\section{RACE/ETHNICITY AND SEX DISTRIBUTION OF PROFESSIONAL STAFF IN ARL UNIVERSITY LIBRARIES FY 2007-2008}

\begin{tabular}{|c|c|c|c|c|c|}
\hline \multicolumn{6}{|c|}{ United States } \\
\hline & \multicolumn{2}{|c|}{ Men } & \multicolumn{2}{|c|}{ Women } & \multirow[t]{2}{*}{ Total } \\
\hline & Number of Staff & Percent of Total & Number of Staff & Percent of Total & \\
\hline Main & 2,794 & $37.32 \%$ & 4,692 & $62.68 \%$ & 7,486 \\
\hline Medical & 224 & $26.54 \%$ & 620 & $73.46 \%$ & 844 \\
\hline Law & 237 & $34.05 \%$ & 459 & $65.95 \%$ & 696 \\
\hline Minority ${ }^{*}$ & 392 & $30.63 \%$ & 888 & $69.38 \%$ & 1,280 \\
\hline Non-minority & 2,863 & $36.96 \%$ & 4,883 & $63.04 \%$ & 7,746 \\
\hline All & 3,255 & $36.06 \%$ & 5,771 & $63.94 \%$ & 9,026 \\
\hline \multicolumn{6}{|c|}{ Canada } \\
\hline & \multicolumn{2}{|c|}{ Men } & \multicolumn{2}{|c|}{ Women } & Total \\
\hline & Number of Staff & Percent of Total & Number of Staff & Percent of Total & \\
\hline Main & 269 & $32.49 \%$ & 559 & $67.51 \%$ & 828 \\
\hline Medical & 11 & $11.83 \%$ & 82 & $88.17 \%$ & 93 \\
\hline Law & 12 & $33.33 \%$ & 24 & $66.67 \%$ & 36 \\
\hline All & 292 & $30.51 \%$ & 665 & $69.49 \%$ & 957 \\
\hline \multicolumn{6}{|c|}{ United States and Canada (Combined) } \\
\hline & \multicolumn{2}{|c|}{ Men } & \multicolumn{2}{|c|}{ Women } & Total \\
\hline & Number of Staff & Percent of Total & Number of Staff & Percent of Total & \\
\hline Main & 3,063 & $36.84 \%$ & 5,251 & $63.16 \%$ & 8,314 \\
\hline Medical & 235 & $24.92 \%$ & 702 & $74.44 \%$ & 937 \\
\hline Law & 249 & $33.47 \%$ & 483 & $64.92 \%$ & 732 \\
\hline All & 3,547 & $35.47 \%$ & 6,436 & $64.35 \%$ & 9,983 \\
\hline
\end{tabular}

\footnotetext{
* Includes staff in medical and law libraries.
}

\section{Gender Data}

Many readers of previous surveys have inquired about evidence of gender-based salary differentials in ARL libraries. Data on salary comparisons for directors also are frequently requested. The average salary 
for male directors was higher than that of their female counterparts (see Table 17); however, the number of women in the top administrative library position has been growing steadily in recent years (62 women directors out of 111 total directorships reported).

Looking at other job categories, though, as Table 17 demonstrates, average salaries for men in most cases still surpass those of women in the same job category. In only nine categories of the 27 used in the table do the average salaries of women exceed those of men. The overall salary for women is $95.39 \%$ that of men for the 113 ARL university libraries, compared to $95.69 \%$ in 2006-2007. This shows a marked closure of the gender gap in ARL libraries over the long term — in 1980-1981, women in ARL libraries made roughly 87\% that of men.

Table 18 provides average years of professional experience for many of the same staffing categories for which salary data are shown in Table 17, revealing that experience differentials between men and women cannot account fully for the salary differentials. Women average more experience in all but two of the nine job categories in which they average higher pay, but there are other categories in which women on average have more experience and less pay: Director, Assistant Director, Functional Specialist, and Department Head--Other. Table 19 further reveals that the average salary for men is consistently higher than the average salary for women in all ten of the experience cohorts, a pattern that is also repeated for minority librarians: the average salary for minority men is higher than that for minority women in nine out of the ten experience cohorts (see Table 30).

There is a sense that the gender gap persists in academe in areas beyond the library and that a renewed commitment to resolve the problem is needed. ${ }^{4}$ A variety of reasons have been offered as to why these trends persist, most notably the perception that work is peripheral in a woman's life and, consequently, female-dominated professions are undervalued. Librarianship is predominantly and persistently a woman's profession. The scarcity of men in the profession has been well documented in many studies — the largest percentage of men employed in ARL libraries was 38.2\% in 1980-1981; since then men have consistently represented about $35 \%$ of the professional staff in ARL libraries.

\section{The Functional Specialist Breakdown}

In 2004, the ARL Statistics and Measurement Committee accepted a proposal from the ACRL Personnel Administrators and Staff Development Officers Discussion Group to break down the Functional Specialist category. The Group's major concern was that so many different types of positions, with their varying job descriptions and salaries, were being labeled with the code FSPEC that data reported for the category were beginning to lose meaning. For each position that would have been labeled FSPEC in past years, the proposal offered ARL institutions two options: either use one of eight new codes to describe that position; or, if none of the eight new codes could adequately describe that position, use FSPEC. As seen in Figure 3a, only 5.8\% of Functional Specialists in all libraries did not use an alternative code, a significant decrease from $20.2 \%$ in 2006-2007. Of the positions that did use an alternate code, 50.5\% of them were Archivists or Information Technology specialists.

\footnotetext{
4 There are many instances citing the continuation of gender inequity in academia. See, for example: Denise K. Manger's articles in the Chronicle of Higher Education, "Faculty Salaries Increased 3.7\% in 1999-2000" (14 Apr. 2000: A20) and "Faculty Salaries are Up 3.6\%, Double the Rate of Inflation" (23 Apr. 1999: A16); D. W. Miller, "Salary Gap Between Male and Female Professors Grows Over the Years, Study Suggests," Chronicle of Higher Education, Today's News, 27 Apr. 2000, <http:// chronicle.com/daily/2000/ 04/2000042702n.htm>; and Yolanda Moses, "Salaries in Academe: The Gender Gap Persists," Chronicle of Higher Education 12 Dec. 1997: A60.
} 
FIGURE 3a

DISTRIBUTION OF JOB SUB-CODES FOR FUNCTIONAL SPECIALIST

\begin{tabular}{|l|r|r|r|r|r|r|r|r|}
\hline \multirow{2}{*}{ Position } & \multicolumn{2}{|c|}{ Main } & \multicolumn{2}{|c|}{ Law } & \multicolumn{2}{|c|}{ Medical } & \multicolumn{2}{|c|}{ All } \\
\cline { 2 - 8 } & No. & Percent & No. & Percent & No. & Percent & No. & Percent \\
\hline Archivist & 333 & $17.0 \%$ & 32 & $23.9 \%$ & 23 & $41.8 \%$ & 388 & $18.0 \%$ \\
Business Manager & 409 & $20.8 \%$ & 16 & $11.9 \%$ & 6 & $10.9 \%$ & 431 & $20.0 \%$ \\
Human Resources & 129 & $6.6 \%$ & 13 & $9.7 \%$ & 2 & $3.6 \%$ & 144 & $6.7 \%$ \\
IT - Programming & 94 & $4.8 \%$ & 1 & $0.7 \%$ & 0 & $0.0 \%$ & 95 & $4.4 \%$ \\
IT - Systems & 406 & $20.7 \%$ & 39 & $29.1 \%$ & 14 & $25.5 \%$ & 459 & $21.3 \%$ \\
IT - Web Development & 129 & $6.6 \%$ & 15 & $11.2 \%$ & 3 & $5.5 \%$ & 147 \\
Media/Multimedia & 246 & $12.5 \%$ & 11 & $8.2 \%$ & 2 & $3.6 \%$ & 259 & $12.0 \%$ \\
Preservation/Conservation & 99 & $5.0 \%$ & 4 & $3.0 \%$ & 2 & $3.6 \%$ & 105 \\
Other Functional Specialists & 118 & $6.0 \%$ & 3 & $2.2 \%$ & 3 & $5.5 \%$ & 124 & $5.9 \%$ \\
\hline Total & $\mathbf{1 , 9 6 3}$ & & $\mathbf{1 3 4}$ & & $\mathbf{5 5} \%$ \\
\hline
\end{tabular}

Figure $3 \mathrm{~b}$, below, displays the average salaries of the subcategories by position and sex in main libraries, in the same fashion as Table 17. The salaries in each of the sub-categories deviate widely about the combined Functional Specialist average salary of $\$ 60,748$. Programming specialists have the highest average of all subcategories, with an average salary of $\$ 69,804$; specialists in Preservation/Conservation have the lowest average salary of $\$ 51,024$.

FIGURE 3b

DISTRIBUTION OF JOB SUB-CODES FOR FUNCTIONAL SPECIALIST

\begin{tabular}{|l|r|r|r|r|r|r|}
\hline \multirow{2}{*}{ Position } & \multicolumn{2}{|c|}{ Women } & \multicolumn{2}{c|}{ Men } & \multicolumn{2}{c|}{ Total } \\
\cline { 2 - 7 } & Salary & No. & Salary & No. & Salary & No. \\
\hline Archivist & $\$ 56,938$ & 225 & 59,334 & 108 & 57,715 & 333 \\
Business Manager & 56,733 & 260 & 60,019 & 149 & 57,930 & 409 \\
Human Resources & 65,462 & 78 & 68,418 & 51 & 66,630 & 129 \\
IT - Programming & 68,929 & 84 & 77,154 & 10 & 69,804 & 94 \\
IT - Systems & 63,941 & 158 & 61,503 & 248 & 62,452 & 406 \\
IT - Web Development & 57,542 & 61 & 60,647 & 68 & 59,179 & 129 \\
Media/Multimedia & 63,411 & 64 & 65,882 & 182 & 65,239 & 246 \\
Preservation/Conservation & 52,690 & 38 & 49,987 & 61 & 51,024 & 99 \\
Other Functional Specialists & 59,029 & 80 & 62,287 & 38 & 60,078 & 118 \\
& & & & & & $\mathbf{9 1 5}$ \\
\hline
\end{tabular}

In regards to the gender gap in ARL libraries explained in the previous section, it is worth noting that the average salaries of men are higher than those of women in seven out of the nine categories in Figure $3 \mathrm{~b}$. 


\section{Institutional Characteristics and Salaries}

\section{A. Public and Private Institutions}

The gap between salaries paid in private U.S. ARL university libraries and those paid in publicly supported U.S. university libraries is $6.2 \%$, or an average of $\$ 4,061$ more paid for a position in a private institution. There are four job categories in which average salaries in the public sector exceeded those in private university libraries: Heads of Circulation, Heads of Documents and Maps, Heads of Rare Books and Manuscripts, and Reference librarians with more than 14 years of experience were paid more on average in public institutions (see Table 21).

\section{B. Library Size}

Library size, as measured by the number of professional staff, is another significant determinant of salary. As a rule, the largest libraries pay the highest average salaries, not only overall, but for specific positions as well. The cutoff staffing levels used to determine the largest cohort of libraries, after declining in every year since 1995-1996, continued to hold steady at 110 in 2007-2008. ${ }^{5}$ The largest libraries, those with more than 110 staff, reported the highest average salary, $\$ 69,603$, compared to $\$ 67,902$ for the libraries with between 75 and 110 staff. The smallest libraries (22-49 staff) had the third-highest average salary of the cohorts, followed by those libraries with 50 to 74 staff. The gap between the highest paying cohort and the lowest paying cohort is $\$ 4,613$, about $22 \%$ smaller than last year's difference of $\$ 5,948$ (see Table 23). The sharp decrease in the gap between highest-paying and lowest-paying cohorts is related to the strengthening of the Canadian dollar relative to the U.S. dollar (see Table 4 for a list of exchange rates since 1984-1985), as 11 of the 14 Canadian ARL members have less than 74 staff.

\section{Geographic Area}

The highest salaries are found in New England (see Table 25), followed by the Pacific and Middle Atlantic regions. All three areas have overall average salaries higher than $\$ 70,000$, with New England averaging as high as $\$ 72,947$. The U.S. / Canadian exchange rate has dropped precipitously over the past four years; as a result, Canada has moved from being the lowest-paying region to the third-highest-paying region, with an average salary in U.S. dollars of $\$ 70,667$. Instead the West South Central region had the lowest average salary, with an average of $\$ 60,600$.

\section{Rank Structure}

Rank structure continues to provide a useful framework for examining professional salaries in ARL university libraries. Figure 4, below, displays average salary and years of experience in the most commonly used rank structures. Readers should be aware that not all individuals have a rank that fits into the rank structure the library utilizes. Most commonly, directors may have no rank or a rank outside the structure, and it is common for non-librarians included in the survey (business officers, personnel staff, computer specialists, etc.) to be unranked, as well.

\footnotetext{
5 In 1995-1996, the largest cohort of libraries was determined based on staff over 124; in 1996-1998, over 120; in 1998-1999, over 115; and since 1999-2000, over 110. See Table 23.
} 
The pattern of relationships between rank and salary seen in past years continues, where higher rank is associated with higher average years of experience and a higher salary. 6,104 of the 9,983 librarians in ARL university member libraries occupy a rank within these three most commonly found ranking systems, and the largest number of professionals $(3,437)$ occupies a rank in a four-step rank structure.

FIGURE 4

AVERAGE SALARIES AND AVERAGE YEARS OF EXPERIENCE OF LIBRARY PROFESSIONALS IN LIBRARIES WITH THREE, FOUR, AND FIVE STEP RANK STRUCTURES

FY 2007-2008

\begin{tabular}{|c|c|c|c|c|c|c|}
\hline & \multicolumn{2}{|c|}{ Three-Step } & \multicolumn{2}{|c|}{ Four-Step } & \multicolumn{2}{|c|}{ Five-Step } \\
\hline & Salary & Experience & Salary & Experience & Salary & Experience \\
\hline Librarian 1 & 52,906 & 8.0 & 50,034 & 7.7 & 47,956 & 9.3 \\
\hline Librarian 2 & 63,819 & 16.3 & 54,505 & 11.4 & 56,907 & 14.5 \\
\hline Librarian 3 & 83,478 & 24.8 & 68,114 & 20.2 & 63,749 & 18.0 \\
\hline Librarian 4 & & & 84,864 & 26.5 & 80,299 & 24.6 \\
\hline Librarian 5 & & & & & 91,577 & 27.2 \\
\hline No. of Staff & \multicolumn{2}{|c|}{1,351} & \multicolumn{2}{|c|}{$\mathbf{3 , 4 3 7}$} & \multicolumn{2}{|c|}{1,127} \\
\hline
\end{tabular}

\section{INFLATION EFFECT}

Tables 2 and 6 reveal changes in beginning professional and median salaries, as well as changes in the U.S. Bureau of Labor's Cost of Living Index (CPI-U) for university and nonuniversity research libraries. Table 3 is similar to Table 2, but reports data only on U.S. libraries. Table 4 shows trend data for Canadian libraries and compares them to the Canadian Consumer Price Index changes. Tables 2, 3, and 4 include law and medical library staff in ARL university libraries. All tables indicate that the purchasing power of professionals working in ARL libraries is keeping up with inflation.

For a second consecutive year, the median U.S. salary has been exceeded by the median Canadian salary converted to U.S. dollars. The median salary for all ARL university libraries was $\$ 61,833$ in 2007-2008; for U.S. libraries only it was $\$ 61,329$; and for Canadian libraries converted into U.S. dollars it was $\$ 67,331$, or a median of $\$ 76,239$ Canadian dollars. In addition to the gains made by the Canadian dollar against the U.S. dollar, Canadian salaries also increased sharply when denominated in Canadian dollars, by 3.9\% (Table 4), while U.S. salaries increased 3.5\% (Table 3). The median salary for combined U.S. and Canadian university libraries increased 3.6\% (Table 2); At the same time, the U.S. Consumer Price Index increased 2.4\% in the last year and the Canadian Consumer Price Index increased 2.5\%.

Beginning salaries in the university sector continue to increase at a steady rate. The median beginning salary in ARL university libraries increased to $\$ 41,125$; this $2.8 \%$ is almost half the $5.5 \%$ increase reported in 2006-2007. After a 10.6\% jump in 2006-2007, the median beginning salary for ARL nonuniversity research libraries increased by only $3.7 \%$ to $\$ 44,359$. Table 6 reveals that the median salary for nonuniversity staff has increased about $0.2 \%$ since $2006-2007$, to $\$ 80,261$.

Libraries need staff with high-level technical skills to operate at the more sophisticated and complex information environments that are in place. As people are hired with higher beginning salaries, the inability 
to adjust the overall salary structure to achieve some equity for the experienced staff members is another factor that contributes to slow salary growth for higher salaries.

Readers are reminded that these data reflect only salaries, and that there are other compensation issues which may have influenced the pattern of salaries in various institutions. In addition, a highly standardized structure for capturing data has been used, which may portray results in a way that cannot be fully representative of a local situation.

Martha Kyrillidou Mark Young Jason Barber Association of Research Libraries August 21, 2008 

SALARY LEVELS FOR STAFF IN ARL LIBRARIES

Tables 1-4 
Figures in columns headed by fiscal year show the number of filled professional positions. Columns headed by cum. \% show the percentage of all filled positions with salaries equal to or more than the beginning of each salary range.

\begin{tabular}{|c|c|c|c|c|c|c|c|c|}
\hline \multirow[b]{2}{*}{ Salary Range } & \multicolumn{4}{|c|}{ University Librarians } & \multicolumn{4}{|c|}{ Nonuniversity Librarians } \\
\hline & $\begin{array}{c}\text { FY } \\
\text { 2006-07 }\end{array}$ & $\begin{array}{c}\text { cum. } \\
\%\end{array}$ & $\begin{array}{c}\text { FY } \\
2007-08\end{array}$ & $\begin{array}{c}\text { cum. } \\
\%\end{array}$ & $\begin{array}{c}\text { FY } \\
\text { 2006-07 }\end{array}$ & $\begin{array}{c}\text { cum. } \\
\%\end{array}$ & $\begin{array}{c}\text { FY } \\
2007-08\end{array}$ & $\begin{array}{c}\text { Cum. } \\
\%\end{array}$ \\
\hline More than 250,000 & 9 & $0.1 \%$ & 12 & $0.1 \%$ & 1 & $0.0 \%$ & 1 & $0.0 \%$ \\
\hline $200,000-250,000$ & 28 & $0.4 \%$ & 30 & $0.4 \%$ & 1 & $0.1 \%$ & 1 & $0.1 \%$ \\
\hline $175,000-199,999$ & 31 & $0.7 \%$ & 41 & $0.8 \%$ & 2 & $0.1 \%$ & 7 & $0.2 \%$ \\
\hline $150,000-174,999$ & 60 & $1.3 \%$ & 81 & $1.6 \%$ & 106 & $2.9 \%$ & 108 & $3.1 \%$ \\
\hline $140,000-149,999$ & 41 & $1.7 \%$ & 37 & $2.0 \%$ & 8 & $3.1 \%$ & 148 & $7.0 \%$ \\
\hline $130,000-139,999$ & 47 & $2.2 \%$ & 57 & $2.6 \%$ & 215 & $8.7 \%$ & 118 & $10.1 \%$ \\
\hline $120,000-129,999$ & 68 & $2.9 \%$ & 90 & $3.5 \%$ & 107 & $11.5 \%$ & 223 & $16.0 \%$ \\
\hline $110,000-119,999$ & 143 & $4.3 \%$ & 177 & $5.3 \%$ & 277 & $18.7 \%$ & 199 & $21.2 \%$ \\
\hline $100,000-109,999$ & 224 & $6.6 \%$ & 246 & $7.7 \%$ & 367 & $28.3 \%$ & 351 & $30.4 \%$ \\
\hline $95,000-99,999$ & 146 & $8.1 \%$ & 212 & $9.8 \%$ & 179 & $33.0 \%$ & 219 & $36.2 \%$ \\
\hline $90,000-94,999$ & 251 & $10.7 \%$ & 299 & $12.8 \%$ & 256 & $39.6 \%$ & 142 & $40.0 \%$ \\
\hline $85,000-89,999$ & 316 & $13.9 \%$ & 374 & $16.6 \%$ & 185 & $44.5 \%$ & 295 & $47.7 \%$ \\
\hline $80,000-84,999$ & 436 & $18.3 \%$ & 469 & $21.3 \%$ & 307 & $52.5 \%$ & 285 & $55.2 \%$ \\
\hline $76,000-79,999$ & 429 & $22.7 \%$ & 456 & $25.9 \%$ & 194 & $57.5 \%$ & 146 & $59.1 \%$ \\
\hline $74,000-75,999$ & 219 & $24.9 \%$ & 229 & $28.1 \%$ & 84 & $59.7 \%$ & 88 & $61.4 \%$ \\
\hline $72,000-73,999$ & 240 & $27.4 \%$ & 330 & $31.5 \%$ & 134 & $63.2 \%$ & 180 & $66.1 \%$ \\
\hline $70,000-71,999$ & 289 & $30.3 \%$ & 306 & $34.5 \%$ & 147 & $67.1 \%$ & 119 & $69.3 \%$ \\
\hline $68,000-69,999$ & 308 & $33.4 \%$ & 312 & $37.6 \%$ & 129 & $70.4 \%$ & 120 & $72.4 \%$ \\
\hline $66,000-67,999$ & 341 & $36.9 \%$ & 408 & $41.7 \%$ & 127 & $73.7 \%$ & 87 & $74.7 \%$ \\
\hline $64,000-65,999$ & 398 & $41.0 \%$ & 363 & $45.4 \%$ & 129 & $77.1 \%$ & 95 & $77.2 \%$ \\
\hline $62,000-63,999$ & 395 & $45.0 \%$ & 436 & $49.7 \%$ & 130 & $80.5 \%$ & 75 & $79.2 \%$ \\
\hline $60,000-61,999$ & 432 & $49.4 \%$ & 397 & $53.7 \%$ & 69 & $82.3 \%$ & 80 & $81.3 \%$ \\
\hline $58,000-59,999$ & 421 & $53.7 \%$ & 494 & $58.7 \%$ & 90 & $84.7 \%$ & 138 & $84.9 \%$ \\
\hline $56,000-57,999$ & 475 & $58.5 \%$ & 472 & $63.4 \%$ & 148 & $88.5 \%$ & 78 & $87.0 \%$ \\
\hline $54,000-55,999$ & 508 & $63.7 \%$ & 511 & $68.5 \%$ & 101 & $91.2 \%$ & 135 & $90.5 \%$ \\
\hline $52,000-53,999$ & 515 & $68.9 \%$ & 510 & $73.6 \%$ & 38 & $92.1 \%$ & 104 & $93.3 \%$ \\
\hline $50,000-51,999$ & 519 & $74.2 \%$ & 500 & $78.6 \%$ & 63 & $93.8 \%$ & 52 & $94.7 \%$ \\
\hline $48,000-49,999$ & 494 & $79.2 \%$ & 459 & $83.2 \%$ & 37 & $94.8 \%$ & 46 & $95.9 \%$ \\
\hline $46,000-47,999$ & 508 & $84.4 \%$ & 440 & $87.6 \%$ & 52 & $96.1 \%$ & 98 & $98.4 \%$ \\
\hline $44,000-45,999$ & 397 & $88.4 \%$ & 384 & $91.5 \%$ & 73 & $98.0 \%$ & 15 & $98.8 \%$ \\
\hline $42,000-43,999$ & 360 & $92.1 \%$ & 321 & $94.7 \%$ & 31 & $98.8 \%$ & 12 & $99.2 \%$ \\
\hline $40,000-41,999$ & 292 & $95.1 \%$ & 217 & $96.9 \%$ & 6 & $99.0 \%$ & 8 & $99.4 \%$ \\
\hline $38,000-39,999$ & 194 & $97.0 \%$ & 132 & $98.2 \%$ & 10 & $99.2 \%$ & 4 & $99.5 \%$ \\
\hline $36,000-37,999$ & 146 & $98.5 \%$ & 92 & $99.1 \%$ & 10 & $99.5 \%$ & 10 & $99.7 \%$ \\
\hline $34,000-35,999$ & 73 & $99.3 \%$ & 41 & $99.5 \%$ & 8 & $99.7 \%$ & 1 & $99.8 \%$ \\
\hline $32,000-33,999$ & 32 & $99.6 \%$ & 24 & $99.8 \%$ & 9 & $99.9 \%$ & 8 & $100.0 \%$ \\
\hline $30,000-31,999$ & 19 & $99.8 \%$ & 14 & $99.9 \%$ & 1 & $100.0 \%$ & 0 & $100.0 \%$ \\
\hline less than 30,000 & 20 & $100.0 \%$ & 10 & $100.0 \%$ & 1 & $100.0 \%$ & 1 & $100.0 \%$ \\
\hline Total Positions & & 9,824 & & 9,983 & & 3,832 & & 3,797 \\
\hline Median Salary & & $\$ 59,648$ & & $\$ 61,833$ & & $\$ 80,124$ & & $\$ 80,261$ \\
\hline
\end{tabular}

18· ARL Annual Salary Survey 2007-2008 
Salary figures for the current year are displayed in the context of the previous years and compared to the changes in the Consumer Price Index (CPI) to show trends in the purchasing power of median and beginning professional salaries. Salary figures and CPI numbers have been converted to adjusted indexes using July 1984 as the base. Actual CPI data retrieved from the U.S. Department of Labor, Bureau of Labor Statistics' Consumer Price Index for All Urban Consumers - (CPI-U), located at http:/ /146.142.4.24/cgi-bin/ surveymost?cu.

\begin{tabular}{ccccccccc}
\hline $\begin{array}{l}\text { Fiscal } \\
\text { Year }\end{array}$ & $\begin{array}{c}\text { No. of } \\
\text { Libs. }\end{array}$ & $\begin{array}{c}\text { Total } \\
\text { Staff }\end{array}$ & $\begin{array}{c}\text { Median } \\
\text { Salary }\end{array}$ & $\begin{array}{c}\text { BPS }^{\ddagger} \\
\text { Median }\end{array}$ & $\begin{array}{c}\text { Median } \\
\text { Salary } \\
\text { Index }\end{array}$ & $\begin{array}{c}\text { BPS } \\
\text { Index }\end{array}$ & $\begin{array}{c}\text { Actual } \\
\text { CPI }\end{array}$ & $\begin{array}{c}\text { Adjusted } \\
\text { CPI }\end{array}$ \\
\hline & & & & & & & & \\
$2007-08$ & 113 & 9,983 & $\$ 61,833$ & $\$ 41,125$ & 236.9 & 249.7 & 208.3 & 200.5 \\
$2006-07$ & 113 & 9,824 & 59,648 & 40,000 & 228.5 & 242.4 & 203.5 & 195.9 \\
$2005-06$ & 113 & 9,655 & 57,074 & 37,920 & 218.7 & 229.8 & 195.4 & 188.1 \\
$2004-05$ & 113 & 9,487 & 55,250 & 36,984 & 211.7 & 224.1 & 189.4 & 182.3 \\
$2003-04$ & 114 & 9,492 & 53,000 & 36,000 & 203.1 & 218.2 & 183.9 & 177.0 \\
$2002-03$ & 114 & 9,469 & 51,636 & 35,000 & 197.8 & 212.1 & 180.1 & 173.3 \\
$2001-02$ & 113 & 9,198 & 50,724 & 34,000 & 194.3 & 206.1 & 177.5 & 170.8 \\
$2000-01$ & 112 & 8,882 & 49,068 & 32,879 & 188.0 & 199.3 & 172.8 & 166.3 \\
$1999-2000$ & 111 & 8,595 & 47,377 & 31,100 & 181.5 & 188.5 & 166.7 & 160.4 \\
$1998-99$ & 110 & 8,400 & 45,775 & 30,000 & 175.2 & 181.7 & 163.2 & 157.1 \\
$1997-98$ & 110 & 8,414 & 44,534 & 28,500 & 170.5 & 172.6 & 160.5 & 154.5 \\
$1996-97$ & 109 & 8,325 & 43,170 & 27,687 & 165.3 & 167.7 & 157.0 & 151.1 \\
$1995-96$ & 108 & 8,231 & 41,901 & 27,000 & 160.5 & 163.6 & 152.5 & 146.8 \\
$1994-95$ & 108 & 8,216 & 41,088 & 26,000 & 157.4 & 157.6 & 148.4 & 142.8 \\
$1993-94$ & 108 & 8,132 & 40,225 & 25,834 & 154.1 & 156.6 & 144.4 & 139.0 \\
$1992-93$ & 108 & 8,212 & 39,265 & 25,000 & 150.4 & 151.5 & 140.2 & 134.9 \\
$1991-92$ & 107 & 8,256 & 38,537 & 24,000 & 147.7 & 145.5 & 136.2 & 131.1 \\
$1990-91$ & 107 & 8,382 & 36,701 & 23,800 & 140.6 & 144.2 & 130.7 & 125.8 \\
$1989-90$ & 107 & 8,253 & 34,629 & 22,000 & 132.7 & 133.3 & 124.0 & 119.3 \\
$1988-89$ & 107 & 8,087 & 32,461 & 20,400 & 124.4 & 123.6 & 118.3 & 113.9 \\
$1987-88$ & 106 & 7,962 & 30,534 & 19,460 & 117.0 & 117.9 & 113.6 & 109.3 \\
$1986-87$ & 105 & 7,718 & 28,941 & 18,250 & 110.9 & 110.6 & 109.6 & 105.5 \\
$1985-86$ & 105 & 7,543 & 27,485 & 17,500 & 105.3 & 106.1 & 107.6 & 103.6 \\
$1984-85$ & 104 & 7,161 & 26,100 & 16,500 & 100.0 & 100.0 & 103.9 & 100.0 \\
\hline
\end{tabular}

* Canadian salaries expressed in U.S. dollars.

$\dagger$ Includes medical and law libraries.

\$ Beginning professional salary. 
Salary figures for the current year are displayed in the context of previous years and compared to the changes in the U.S. Consumer Price Index (CPI) to show trends in the purchasing power of median and beginning professional salaries. Salary figures and CPI numbers have been converted to adjusted indexes, using July 1984 as the base. Actual CPI data retrieved from the U.S. Department of Labor, Bureau of Labor Statistics' Consumer Price Index for All Urban Consumers - (CPI-U), at http://146.142.4.24/ cgibin/surveymost?cu.

\begin{tabular}{|c|c|c|c|c|c|c|c|c|}
\hline $\begin{array}{l}\text { Fiscal } \\
\text { Year }\end{array}$ & $\begin{array}{l}\text { No. of } \\
\text { Libs. }\end{array}$ & $\begin{array}{l}\text { Total } \\
\text { Staff }\end{array}$ & $\begin{array}{c}\text { Median } \\
\text { Salary }\end{array}$ & $\begin{array}{c}\text { Median } \\
\text { Salary } \\
\text { Change }\end{array}$ & $\begin{array}{c}\text { Median } \\
\text { Salary } \\
\text { Index }\end{array}$ & $\begin{array}{c}\text { Actual } \\
\text { CPI }\end{array}$ & $\begin{array}{l}\text { Adjusted } \\
\text { CPI }\end{array}$ & $\begin{array}{c}\text { CPI } \\
\text { Change }\end{array}$ \\
\hline 2007-08 & 99 & 9,026 & $\$ 61,329$ & $3.5 \%$ & 236.4 & 208.3 & 200.5 & $2.4 \%$ \\
\hline 2006-07 & 99 & 8,866 & 59,280 & 3.7 & 228.5 & 203.5 & 195.9 & 4.1 \\
\hline 2005-06 & 99 & 8,700 & 57,173 & 2.8 & 220.4 & 195.4 & 188.1 & 3.2 \\
\hline 2004-05 & 99 & 8,581 & 55,600 & 3.2 & 214.3 & 189.4 & 182.3 & 3.0 \\
\hline 2003-04 & 100 & 8,581 & 53,859 & 2.0 & 207.6 & 183.9 & 177.0 & 2.1 \\
\hline 2002-03 & 100 & 8,544 & 52,789 & 1.9 & 203.5 & 180.1 & 173.3 & 1.5 \\
\hline 2001-02 & 99 & 8,337 & 51,806 & 4.1 & 199.7 & 177.5 & 170.8 & 2.7 \\
\hline 2000-01 & 99 & 8,127 & 49,753 & 3.7 & 191.8 & 172.8 & 166.3 & 3.7 \\
\hline 1999-2000 & 98 & 7,858 & 48,000 & 4.1 & 185.0 & 166.7 & 160.4 & 2.1 \\
\hline 1998-99 & 97 & 7,671 & 46,130 & 3.6 & 177.8 & 163.2 & 157.1 & 1.7 \\
\hline 1997-98 & 97 & 7,682 & 44,544 & 3.4 & 171.7 & 160.5 & 154.5 & 2.2 \\
\hline 1996-97 & 96 & 7,562 & 43,084 & 3.4 & 166.1 & 157.0 & 151.1 & 3.0 \\
\hline 1995-96 & 95 & 7,435 & 41,651 & 2.7 & 160.5 & 152.5 & 146.8 & 2.8 \\
\hline 1994-95 & 95 & 7,401 & 40,573 & 3.4 & 156.4 & 148.4 & 142.8 & 2.8 \\
\hline 1993-94 & 95 & 7,390 & 39,257 & 3.0 & 151.3 & 144.4 & 139.0 & 3.0 \\
\hline 1992-93 & 95 & 7,375 & 38,124 & 3.0 & 146.9 & 140.2 & 134.9 & 2.9 \\
\hline 1991-92 & 94 & 7,408 & 37,009 & 3.5 & 142.6 & 136.2 & 131.1 & 4.2 \\
\hline 1990-91 & 94 & 7,543 & 35,761 & 5.2 & 137.8 & 130.7 & 125.8 & 5.4 \\
\hline 1989-90 & 94 & 7,344 & 34,000 & 5.8 & 131.0 & 124.0 & 119.3 & 4.8 \\
\hline 1988-89 & 94 & 7,252 & 32,149 & 5.4 & 123.9 & 118.3 & 113.9 & 4.1 \\
\hline 1987-88 & 93 & 7,145 & 30,492 & 5.1 & 117.5 & 113.6 & 109.3 & 3.6 \\
\hline 1986-87 & 92 & 6,886 & 29,021 & 6.5 & 111.9 & 109.6 & 105.5 & 1.9 \\
\hline 1985-86 & 91 & 6,707 & 27,249 & 5.0 & 105.0 & 107.6 & 103.6 & 3.6 \\
\hline 1984-85 & 91 & 6,456 & 25,946 & 6.9 & 100.0 & 103.9 & 100.0 & - \\
\hline
\end{tabular}

* Includes medical and law libraries in median salary. 
Salary figures for the current year are displayed in the context of previous years. Canadian salaries are presented in both U.S. \$ and Canadian $\$$ denominations and the annual exchange rate used in the salary surveys is also listed. Canadian salaries are also compared to the changes in the Canadian Consumer Price Index (CPI) to show trends in the purchasing power of median Canadian salaries. CPI number changes are based on July CPI figures. The Canadian CPI change is indicated in the August 22, 2006 edition of The Daily, a Statistics Canada publication, at http: / / www.statcan.ca/ english/Subjects/Cpi/ cpi-en.htm.

\begin{tabular}{|c|c|c|c|c|c|c|c|c|}
\hline $\begin{array}{l}\text { Fiscal } \\
\text { Year }\end{array}$ & $\begin{array}{l}\text { No. of } \\
\text { Libs. }\end{array}$ & $\begin{array}{l}\text { Total } \\
\text { Staff }\end{array}$ & $\begin{array}{l}\text { Median } \\
\text { Salary } \\
\text { in U.S. } \$^{\dagger}\end{array}$ & $\begin{array}{c}\text { Median } \\
\text { Salary } \\
\text { Change }^{\dagger}\end{array}$ & $\begin{array}{c}\text { Exchange } \\
\text { Rate }\end{array}$ & $\begin{array}{c}\text { Median } \\
\text { Salary } \\
\text { in Can. \$ }\end{array}$ & $\begin{array}{c}\text { Median } \\
\text { Salary } \\
\text { Change }\end{array}$ & $\begin{array}{c}\text { Canadian } \\
\text { CPI } \\
\text { Change }\end{array}$ \\
\hline 2007-08 & 14 & 957 & $\$ 67,331$ & $6.7 \%$ & 1.13230 & $\$ 76,239$ & $3.9 \%$ & $2.5 \%$ \\
\hline 2006-07 & 14 & 958 & 63,112 & 11.8 & 1.16289 & 73,392 & 4.0 & 2.4 \\
\hline 2005-06 & 14 & 955 & 56,474 & 7.1 & 1.24971 & 70,576 & -0.3 & 2.0 \\
\hline 2004-05 & 14 & 906 & 52,707 & 16.3 & 1.34328 & 70,800 & 3.5 & 2.3 \\
\hline 2003-04 & 14 & 911 & 45,310 & 6.2 & 1.51023 & 68,429 & 2.3 & 2.2 \\
\hline 2002-03 & 14 & 925 & 42,657 & -0.6 & 1.56878 & 66,919 & 2.6 & 2.1 \\
\hline 2001-02 & 14 & 861 & 42,928 & -1.1 & 1.51919 & 65,215 & 2.1 & 2.6 \\
\hline 2000-01 & 13 & 755 & 43,394 & 5.0 & 1.47192 & 63,873 & 2.4 & 3.0 \\
\hline 1999-2000 & 13 & 737 & 41,316 & -3.8 & 1.5103 & 62,400 & 2.4 & 1.8 \\
\hline 1998-99 & 13 & 729 & 42,963 & -2.7 & 1.4177 & 60,909 & 0.9 & 1.0 \\
\hline 1997-98 & 13 & 732 & 44,167 & 1.4 & 1.3663 & 60,346 & 1.7 & 1.8 \\
\hline 1996-97 & 13 & 764 & 43,569 & 0.9 & 1.3613 & 59,310 & -0.4 & 1.2 \\
\hline 1995-96 & 13 & 796 & 43,173 & -1.7 & 1.3794 & 59,554 & 1.3 & 2.5 \\
\hline 1994-95 & 13 & 815 & 43,919 & -6.0 & 1.3381 & 58,768 & 0.7 & 0.2 \\
\hline 1993-94 & 13 & 816 & 46,744 & -4.3 & 1.2488 & 58,374 & 2.9 & 1.6 \\
\hline 1992-93 & 13 & 837 & 48,820 & 2.7 & 1.1623 & 56,744 & 3.4 & 1.3 \\
\hline 1991-92 & 13 & 847 & 47,519 & 5.5 & 1.1547 & 54,870 & 3.6 & 5.8 \\
\hline 1990-91 & 13 & 839 & 45,023 & 15.1 & 1.1759 & 52,942 & 12.5 & 4.2 \\
\hline 1989-90 & 13 & 853 & 39,117 & 12.3 & 1.2026 & 47,042 & 5.3 & 5.4 \\
\hline 1988-89 & 13 & 837 & 34,826 & 11.7 & 1.2826 & 44,668 & 5.3 & 3.8 \\
\hline 1987-88 & 13 & 817 & 31,178 & 10.9 & 1.3602 & 42,408 & 9.1 & 4.7 \\
\hline 1986-87 & 13 & 831 & 28,123 & -1.9 & 1.3817 & 38,858 & 1.2 & 4.2 \\
\hline 1985-86 & 13 & 829 & 28,666 & 1.1 & 1.3388 & 38,378 & 7.9 & 3.8 \\
\hline $1984-85$ & 12 & 705 & 28,346 & -0.8 & 1.2548 & 35,569 & 0.8 & 4.2 \\
\hline
\end{tabular}

* Canadian salaries expressed in U.S. dollars.

$\dagger$ Includes medical and law libraries in median salary. 

ARL NONUNIVERSITY LIBRARIES

Tables 5-6 


\section{TABLE 5: MEDIAN AND BEGINNING PROFESSIONAL SALARIES IN ARL NONUNIVERSITY LIBRARIES*}

\section{Median Salaries}

No. of Staff

FY 2006-07

FY 2007-08

FY 2006-07

FY 2007-08

\begin{tabular}{l} 
Boston Public Library \\
Canada Institute for Scientific \\
and Technical Information * \\
Center for Research Libraries \\
Library of Congress ${ }^{\dagger}$ \\
National Agricultural Library ${ }^{\dagger}$ \\
Library \& Archives of Canada * \\
National Library of Medicine \\
New York Public Library \\
New York State Library \\
Smithsonian Library \\
\hline Canadian salaries expressed in U.S. dollars. \\
${ }^{\dagger}$ See footnotes.
\end{tabular}

$\begin{array}{lrrrr}183 & \$ 56,406 & \$ 58,099 & \$ 37,907 & \$ 37,907 \\ 190 & 59,751 & 61,365 & 40,040 & 44,105\end{array}$

\begin{tabular}{rllll}
31 & 48,076 & 51,000 & 39,000 & 44,584 \\
2,625 & 87,664 & 89,985 & 44,856 & 46,041 \\
100 & 73,720 & 75,671 & 44,856 & 46,041 \\
119 & 53,881 & 55,337 & 46,967 & 44,133 \\
191 & 82,391 & 80,123 & 44,856 & 46,041 \\
239 & 54,478 & 58,674 & 35,912 & 39,295 \\
64 & 59,000 & 58,849 & 40,673 & 42,693 \\
55 & 73,720 & 75,671 & 44,856 & 46,041 \\
\hline
\end{tabular}

$\dagger$ See footnotes. 
Salary figures for the current year are displayed in the context of the previous years and compared to the changes in the Consumer Price Index (CPI) to show trends in the purchasing power of median and beginning professional salaries. Salary figures and CPI numbers have been converted to adjusted indexes, using July 1984 as the base. Actual CPI data retrieved from the U.S. Department of Labor, Bureau of Labor Statistics' Consumer Price Index for All Urban Consumers - (CPI-U), located at http:/ /146.142.4.24/ cgi-bin/ surveymost?cu.

\begin{tabular}{|c|c|c|c|c|c|c|c|c|}
\hline $\begin{array}{l}\text { Fiscal } \\
\text { Year }\end{array}$ & $\begin{array}{l}\text { No. of } \\
\text { Libs. }\end{array}$ & Total Staff & $\begin{array}{l}\text { Median } \\
\text { Salary* }\end{array}$ & $\begin{array}{c}\text { BPS }^{\dagger} \\
\text { Median }\end{array}$ & $\begin{array}{c}\text { Median } \\
\text { Salary Index }\end{array}$ & $\begin{array}{l}\text { BPS }^{\dagger} \\
\text { Index }\end{array}$ & $\begin{array}{c}\text { Actual } \\
\text { CPI }\end{array}$ & $\begin{array}{c}\text { Adjusted } \\
\text { CPI }\end{array}$ \\
\hline $2007-08$ & 10 & 3,797 & $\$ 80,261$ & $\$ 44,359$ & 236.9 & 268.7 & 208.3 & 200.5 \\
\hline $2006-07$ & 10 & 3,832 & 80,124 & 42,765 & 236.5 & 259.1 & 203.5 & 195.9 \\
\hline 2005-06 & 10 & 3,921 & 76,083 & 38,673 & 224.6 & 234.3 & 195.4 & 188.1 \\
\hline 2004-05 & 10 & 3,946 & 74,022 & 34,764 & 218.5 & 210.6 & 189.4 & 182.3 \\
\hline 2003-04 & 10 & 3,877 & 70,020 & 34,739 & 206.8 & 210.4 & 183.9 & 177.0 \\
\hline $2002-03$ & 10 & 3,804 & 65,289 & 34,739 & 192.7 & 210.4 & 180.1 & 173.3 \\
\hline 2001-02 & 10 & 3,717 & 65,025 & 34,389 & 191.9 & 208.3 & 177.5 & 170.8 \\
\hline 2000-01 & 10 & 3,731 & 62,521 & 31,774 & 184.5 & 192.5 & 172.8 & 166.3 \\
\hline 1999-2000 & 10 & 3,737 & 59,916 & 30,849 & 176.8 & 186.9 & 166.7 & 160.4 \\
\hline 1998-99 & 11 & 3,819 & 56,000 & 29,877 & 165.3 & 181.0 & 163.2 & 157.1 \\
\hline $1997-98$ & 11 & 3,779 & 55,055 & 28,724 & 162.5 & 174.0 & 160.5 & 154.5 \\
\hline $1996-97$ & 11 & 3,799 & 51,150 & 28,380 & 151.0 & 172.0 & 157.0 & 151.1 \\
\hline 1995-96 & 11 & 3,915 & 49,149 & 28,162 & 145.1 & 170.7 & 152.5 & 146.8 \\
\hline 1994-95 & 11 & 3,837 & 47,997 & 27,813 & 141.7 & 168.6 & 148.4 & 142.8 \\
\hline 1993-94 & 11 & 4,003 & 44,949 & 26,806 & 132.7 & 162.5 & 144.4 & 139.0 \\
\hline $1992-93$ & 11 & 4,172 & 43,876 & 23,500 & 129.6 & 142.4 & 140.2 & 134.9 \\
\hline 1991-92 & 11 & 2,906 & 42,455 & 23,500 & 125.4 & 142.4 & 136.2 & 131.1 \\
\hline 1990-91 & 12 & 1,363 & 36,013 & 20,800 & 106.3 & 126.1 & 130.7 & 125.8 \\
\hline 1989-90 & 11 & 3,767 & 40,106 & 20,195 & 118.4 & 122.4 & 124.0 & 119.3 \\
\hline 1988-89 & 11 & 3,781 & 37,544 & 19,100 & 110.9 & 115.8 & 118.3 & 113.9 \\
\hline $1987-88$ & 11 & 3,765 & 36,250 & 18,405 & 107.0 & 111.5 & 113.6 & 109.3 \\
\hline $1986-87$ & 10 & 2,790 & 33,020 & 17,912 & 97.5 & 108.6 & 109.6 & 105.5 \\
\hline $1985-86$ & 12 & 3,874 & 33,720 & 17,308 & 99.6 & 104.9 & 107.6 & 103.6 \\
\hline 1984-85 & 11 & 3,840 & 33,863 & 16,500 & 100.0 & 100.0 & 103.9 & 100.0 \\
\hline
\end{tabular}

* Canadian salaries expressed in U.S. dollars.

${ }^{\dagger}$ Beginning professional salary. 

ARL UNIVERSITY LIBRARIES

Tables 7-25 


\section{TABLE 7: FILLED POSITIONS; AVERAGE, MEDIAN, BEGINNING PROFESSIONAL SALARIES; AND AVERAGE YEARS OF EXPERIENCE ARL UNIVERSITY LIBRARIES, FY 2007-2008*}

\begin{tabular}{|c|c|c|c|c|c|c|c|c|}
\hline \multirow[b]{2}{*}{ Institution } & \multirow{2}{*}{$\begin{array}{l}\text { Filled Pos. } \\
\text { FY } 2008\end{array}$} & \multicolumn{2}{|c|}{$\begin{array}{l}\text { Average } \\
\text { Salaries }\end{array}$} & \multicolumn{2}{|c|}{$\begin{array}{l}\text { Median } \\
\text { Salaries }\end{array}$} & \multicolumn{2}{|c|}{$\begin{array}{c}\text { Beginning } \\
\text { Salaries }\end{array}$} & \multirow{2}{*}{$\begin{array}{l}\text { Average } \\
\text { Yrs. Exp. } \\
\text { FY } 2008\end{array}$} \\
\hline & & FY 2007 & FY 2008 & FY 2007 & FY 2008 & FY 2007 & FY 2008 & \\
\hline Alabama * & 57 & $\$ 54,096$ & $\$ 58,232$ & $\$ 49,428$ & $\$ 52,891$ & $\$ 37,000$ & $\$ 40,000$ & 14.8 \\
\hline Alberta ${ }^{\dagger *}$ & 67 & 69,228 & 74,787 & 76,714 & 82,536 & 40,449 & 42,997 & 17.3 \\
\hline Arizona : & 64 & 63,916 & 64,731 & 58,917 & 60,136 & 43,474 & 47,325 & 17.0 \\
\hline Arizona State & 72 & 60,315 & 64,325 & 56,743 & 62,333 & 43,000 & 43,000 & 17.1 \\
\hline Auburn ${ }^{*}$ & 44 & 58,800 & 61,104 & 59,200 & 58,540 & 44,700 & 44,720 & 15.8 \\
\hline Boston University & 51 & 52,505 & 54,472 & 50,000 & 51,450 & 24,700 & 40,800 & 17.7 \\
\hline Boston College & 60 & 64,187 & 67,847 & 61,750 & 65,293 & 39,850 & 41,050 & 19.0 \\
\hline Brigham Young * & 113 & 60,562 & 61,929 & 58,500 & 60,200 & 44,000 & 46,000 & 17.3 \\
\hline British Columbia $^{\dagger}$ & 83 & 63,845 & 69,414 & 62,938 & 68,737 & 43,718 & 45,784 & 15.3 \\
\hline Brown ${ }^{\ddagger}$ & 65 & 62,460 & 65,442 & 58,193 & 61,998 & 37,000 & 38,000 & 20.0 \\
\hline California, Berkeley & 101 & 75,968 & 80,749 & 72,558 & 79,108 & 39,000 & 40,008 & 18.6 \\
\hline California, Davis & 46 & 69,196 & 73,853 & 71,916 & 79,968 & 39,000 & 40,008 & 19.3 \\
\hline California, Irvine & 56 & 72,485 & 71,572 & 73,537 & 73,356 & 39,000 & 40,008 & 14.9 \\
\hline California, Los Angeles * & 140 & 74,082 & 76,029 & 72,084 & 73,382 & 39,000 & 40,008 & 16.9 \\
\hline California, Riverside & 60 & 66,183 & 69,120 & 65,220 & 68,000 & 42,204 & 42,204 & 17.1 \\
\hline California, San Diego ${ }^{\star}$ & 84 & 71,287 & 74,176 & 70,350 & 73,356 & 40,008 & 40,008 & 15.8 \\
\hline California, Santa Barbara ${ }^{\ddagger}$ & 58 & 68,946 & 70,227 & 65,448 & 66,756 & 39,000 & 42,008 & 16.8 \\
\hline Case Western Reserve ${ }^{\ddagger}$ & 37 & 54,258 & 57,089 & 52,842 & 54,335 & 35,000 & 35,000 & 15.3 \\
\hline Chicago $\$$ & 71 & 70,385 & 72,179 & 65,792 & 68,418 & 45,500 & 46,800 & 17.7 \\
\hline Cincinnati & 53 & 62,957 & 65,283 & 58,987 & 61,552 & 37,000 & 38,000 & 21.8 \\
\hline Colorado ${ }^{*}$ & 43 & 59,930 & 65,510 & 57,622 & 61,431 & 41,000 & 41,000 & 17.6 \\
\hline Colorado State & 51 & 62,371 & 62,333 & 60,828 & 60,650 & 40,000 & 43,500 & 14.2 \\
\hline Columbia ${ }^{\ddagger}$ & 189 & 70,854 & 70,838 & 64,160 & 61,585 & 49,500 & 51,500 & 15.6 \\
\hline Connecticut * & 64 & 72,674 & 76,946 & 67,825 & 74,661 & 48,000 & 48,000 & 17.3 \\
\hline Cornell ${ }^{*}$ & 111 & 66,479 & 68,228 & 60,050 & 62,490 & 43,300 & 45,000 & 16.8 \\
\hline Dartmouth & 52 & 68,775 & 71,642 & 65,563 & 71,597 & 38,000 & 40,000 & 16.9 \\
\hline Delaware & 60 & 66,598 & 69,033 & 64,130 & 64,235 & 39,000 & 43,600 & 16.5 \\
\hline Duke ${ }^{\star}$ & 137 & 58,918 & 60,715 & 55,400 & 55,650 & 36,850 & 43,000 & 15.0 \\
\hline Emory ${ }^{\star}$ & 78 & 61,950 & 64,551 & 58,925 & 61,000 & 37,000 & 38,000 & 14.2 \\
\hline Florida : & 74 & 55,761 & 56,403 & 51,413 & 52,327 & 42,000 & 42,000 & 13.6 \\
\hline Florida State & 53 & 46,853 & 48,656 & 42,479 & 45,752 & 41,000 & 42,000 & 12.1 \\
\hline George Washington & 38 & 63,878 & 64,950 & 57,907 & 59,401 & 42,000 & 42,000 & 13.8 \\
\hline Georgetown & 50 & 63,686 & 66,689 & 59,375 & 61,508 & 45,000 & 45,000 & 19.0 \\
\hline Georgia * & 68 & 54,062 & 57,082 & 47,731 & 49,634 & 32,500 & 35,000 & 17.2 \\
\hline Georgia Tech & 40 & 59,700 & 58,753 & 53,732 & 54,631 & 44,000 & 42,000 & 15.7 \\
\hline Guelph ${ }^{\dagger}$ & 48 & 62,335 & 62,069 & 58,833 & 58,271 & 37,574 & 38,590 & 17.2 \\
\hline Harvard ${ }^{*}$ & 464 & 70,160 & 72,861 & 64,443 & 67,486 & 45,500 & 47,100 & 16.7 \\
\hline Hawaii * & 80 & 62,760 & 61,330 & 60,959 & 60,354 & 38,356 & 42,026 & 14.9 \\
\hline Houston * & 40 & 56,901 & 56,608 & 52,799 & 49,037 & 39,000 & 40,000 & 15.2 \\
\hline Howard & 29 & 50,032 & 53,211 & 47,105 & 53,359 & 35,409 & 35,049 & 25.1 \\
\hline Illinois, Chicago & 50 & 58,010 & 58,925 & 52,981 & 54,885 & 42,000 & 47,000 & 16.1 \\
\hline Illinois, Urbana & 144 & 62,401 & 64,009 & 56,091 & 58,247 & 43,000 & 44,500 & 13.1 \\
\hline Indiana ${ }^{\ddagger}$ & 79 & 60,776 & 64,257 & 57,123 & 59,705 & 36,952 & 40,000 & 19.8 \\
\hline Iowa & 66 & 60,666 & 63,234 & 54,207 & 55,700 & 40,000 & 40,000 & 18.3 \\
\hline Iowa State & 56 & 58,887 & 59,891 & 56,054 & 57,452 & 41,000 & 42,500 & 18.3 \\
\hline Johns Hopkins & 75 & 63,555 & 65,765 & 59,795 & 60,060 & 46,700 & 48,335 & 15.6 \\
\hline Kansas & 84 & 57,251 & 59,070 & 54,982 & 55,120 & 40,000 & 40,000 & 14.9 \\
\hline Kent State: & 67 & 54,156 & 56,086 & 51,333 & 53,777 & 51,333 & 43,389 & 15.0 \\
\hline Kentucky & 60 & 56,371 & 59,613 & 54,352 & 57,817 & 37,500 & 38,500 & 20.9 \\
\hline $\mathrm{Laval}^{\dagger \ddagger}$ & 61 & 54,114 & 59,630 & 55,855 & 62,715 & 36,704 & 40,025 & 16.6 \\
\hline Louisiana State & 51 & 47,812 & 48,711 & 43,705 & 44,879 & 36,000 & 38,000 & 13.7 \\
\hline Louisville & 34 & 63,179 & 65,230 & 64,134 & 61,986 & 36,000 & 37,000 & 21.5 \\
\hline McGill $^{\dagger+}$ & 53 & 63,903 & 66,010 & 66,226 & 64,728 & 39,557 & 40,625 & 18.5 \\
\hline McMaster ${ }^{\dagger \ddagger}$ & 22 & 61,406 & 67,549 & 61,044 & 69,173 & 36,311 & 38,598 & 18.9 \\
\hline Manitoba ${ }^{\dagger+}$ & 45 & 72,277 & 77,261 & 74,814 & 79,159 & 38,833 & 41,331 & 23.8 \\
\hline Maryland * & 97 & 61,723 & 64,926 & 59,395 & 61,930 & 40,000 & 42,000 & 18.1 \\
\hline Massachusetts * & 60 & 67,202 & 69,319 & 69,658 & 69,746 & 39,544 & 40,730 & 16.4 \\
\hline
\end{tabular}




\section{TABLE 7: FILLED POSITIONS; AVERAGE, MEDIAN, BEGINNING PROFESSIONAL SALARIES; AND AVERAGE YEARS OF EXPERIENCE ARL UNIVERSITY LIBRARIES, FY 2007-2008*}

\begin{tabular}{|c|c|c|c|c|c|c|c|c|}
\hline \multirow[b]{2}{*}{ Institution } & \multirow{2}{*}{$\begin{array}{l}\text { Filled Pos. } \\
\text { FY } 2008\end{array}$} & \multicolumn{2}{|c|}{$\begin{array}{l}\text { Average } \\
\text { Salaries }\end{array}$} & \multicolumn{2}{|c|}{$\begin{array}{l}\text { Median } \\
\text { Salaries }\end{array}$} & \multicolumn{2}{|c|}{$\begin{array}{l}\text { Beginning } \\
\text { Salaries }\end{array}$} & \multirow{2}{*}{$\begin{array}{c}\text { Average } \\
\text { Yrs. Exp. } \\
\text { FY } 2008\end{array}$} \\
\hline & & FY 2007 & FY 2008 & FY 2007 & FY 2008 & FY 2007 & FY 2008 & \\
\hline MIT & 87 & $\$ 67,285$ & $\$ 69,719$ & $\$ 64,100$ & $\$ 65,650$ & $\$ 45,000$ & $\$ 48,000$ & 16.0 \\
\hline Miami & 39 & 63,695 & 65,171 & 56,863 & 60,000 & 41,700 & 45,000 & 16.6 \\
\hline Michigan & 111 & 64,295 & 66,608 & 59,920 & 63,413 & 40,000 & 41,000 & 18.7 \\
\hline Michigan State & 68 & 62,101 & 61,743 & 59,700 & 57,000 & 47,000 & 47,000 & 16.1 \\
\hline Minnesota & 104 & 64,164 & 66,067 & 61,033 & 63,532 & 38,000 & 40,000 & 18.6 \\
\hline Missouri & 35 & 52,090 & 57,607 & 49,134 & 50,442 & 34,000 & 38,500 & 20.9 \\
\hline Montreal ${ }^{\dagger \ddagger}$ & 84 & 56,909 & 60,262 & 54,360 & 58,216 & 34,504 & 36,144 & 14.9 \\
\hline Nebraska * & 47 & 58,792 & 61,376 & 52,928 & 54,624 & 40,500 & 45,000 & 19.1 \\
\hline New Mexico ${ }^{\star}$ & 32 & 70,749 & 76,612 & 71,319 & 74,768 & 40,000 & 40,000 & 20.7 \\
\hline New York University & 54 & 72,220 & 77,595 & 63,700 & 68,882 & 49,500 & 51,000 & 18.1 \\
\hline North Carolina ${ }^{\ddagger}$ & 92 & 63,189 & 67,938 & 60,000 & 62,825 & 42,000 & 42,000 & 19.8 \\
\hline North Carolina State & 84 & 66,529 & 68,461 & 59,500 & 61,717 & 46,000 & 47,000 & 10.8 \\
\hline Northwestern & 76 & 62,941 & 65,234 & 60,144 & 61,203 & 37,000 & 39,000 & 17.2 \\
\hline Notre Dame ${ }^{\ddagger}$ & 54 & 64,821 & 66,827 & 63,766 & 65,769 & 37,250 & 40,000 & 18.7 \\
\hline Ohio University & 50 & 48,815 & 48,853 & 44,263 & 45,010 & 35,000 & 36,000 & 11.2 \\
\hline Ohio State ${ }^{\ddagger}$ & 123 & 57,544 & 56,745 & 54,834 & 52,941 & 40,500 & 41,500 & 14.5 \\
\hline Oklahoma & 41 & 49,386 & 53,241 & 46,162 & 50,823 & 40,000 & 40,000 & 13.8 \\
\hline Oklahoma State & 60 & 53,406 & 54,593 & 49,536 & 49,824 & 36,000 & 36,000 & 16.6 \\
\hline Oregon ${ }^{*}$ & 48 & 51,535 & 54,999 & 48,424 & 51,176 & 35,000 & 35,000 & 14.9 \\
\hline Pennsylvania * & 93 & 62,553 & 63,029 & 59,665 & 58,526 & 42,000 & 42,500 & 15.4 \\
\hline Pennsylvania State & 136 & 64,546 & 66,725 & 61,308 & 62,730 & 40,000 & 41,200 & 18.8 \\
\hline Pittsburgh ${ }^{\ddagger}$ & 61 & 61,695 & 64,047 & 56,345 & 58,451 & 34,000 & 34,000 & 16.9 \\
\hline Princeton & 120 & 70,082 & 74,179 & 64,600 & 70,000 & 40,000 & 45,000 & 20.2 \\
\hline Purdue ${ }^{\ddagger}$ & 67 & 61,429 & 65,312 & 54,330 & 57,815 & 43,000 & 43,500 & 17.5 \\
\hline Queen`s ${ }^{\dagger \ddagger}$ & 34 & 67,488 & 72,268 & 68,700 & 74,078 & 38,452 & 40,695 & 18.7 \\
\hline Rice & 58 & 58,302 & 61,662 & 51,600 & 54,900 & 36,099 & 39,060 & 15.7 \\
\hline Rochester * & 70 & 53,255 & 55,411 & 49,733 & 51,282 & 36,700 & 36,700 & 15.5 \\
\hline Rutgers ${ }^{*}$ & 91 & 77,298 & 82,023 & 80,048 & 84,829 & 44,525 & 46,781 & 20.1 \\
\hline Saskatchewan ${ }^{\dagger}$ & 39 & 64,936 & 74,526 & 64,350 & 74,456 & 35,951 & 41,598 & 17.0 \\
\hline South Carolina : & 54 & 51,060 & 51,457 & 47,388 & 47,983 & 34,000 & 34,000 & 16.2 \\
\hline Southern California * & 89 & 68,457 & 70,463 & 62,900 & 64,335 & 45,000 & 45,000 & 17.9 \\
\hline Southern Illinois ${ }^{\ddagger}$ & 38 & 53,791 & 54,562 & 47,311 & 49,375 & 39,500 & 41,000 & 14.2 \\
\hline SUNY Albany & 55 & 58,663 & 60,304 & 56,376 & 56,625 & 38,500 & 38,000 & 19.3 \\
\hline SUNY Buffalo * & 76 & 58,519 & 64,414 & 53,790 & 60,852 & 45,000 & 45,000 & 18.6 \\
\hline SUNY Stony Brook & 28 & 68,185 & 70,027 & 65,764 & 67,828 & 42,000 & 43,000 & 22.0 \\
\hline Syracuse ${ }^{\ddagger}$ & 45 & 62,119 & 65,131 & 55,757 & 58,263 & U/A & & 20.5 \\
\hline Temple & 38 & 63,282 & 65,552 & 58,670 & 56,572 & 38,000 & 39,000 & 18.3 \\
\hline Tennessee & 42 & 63,432 & 68,333 & 62,319 & 65,394 & 40,000 & 42,000 & 17.3 \\
\hline Texas ${ }^{\ddagger}$ & 120 & 62,185 & 63,467 & 56,619 & 57,809 & 44,000 & 45,000 & 17.4 \\
\hline Texas A\&M & 98 & 56,327 & 60,024 & 50,502 & 54,857 & 42,000 & 44,000 & 14.7 \\
\hline Texas Tech * & 48 & 51,858 & 59,987 & 47,410 & 53,079 & 38,000 & 45,000 & 12.3 \\
\hline Toronto ${ }^{\dagger \ddagger}$ & 135 & 74,374 & 76,710 & 77,290 & 78,443 & 41,878 & 43,010 & 17.2 \\
\hline Tulane & 28 & 59,287 & 59,023 & 58,294 & 55,664 & 37,000 & 37,000 & 18.1 \\
\hline Utah & 56 & 56,452 & 59,993 & 51,633 & 54,614 & 40,000 & 42,000 & 19.3 \\
\hline Vanderbilt $¥$ & 61 & 56,839 & 57,775 & 51,264 & 52,479 & 38,000 & 39,000 & 17.3 \\
\hline Virginia & 65 & 64,777 & 67,602 & 61,800 & 60,000 & 41,800 & 43,000 & 16.9 \\
\hline Virginia Tech & 33 & 59,256 & 62,691 & 56,150 & 59,672 & 37,000 & 38,500 & 17.9 \\
\hline Washington ${ }^{\star}$ & 118 & 60,054 & 63,655 & 55,656 & 58,020 & 40,000 & 41,000 & 18.9 \\
\hline Washington State & 45 & 58,761 & 58,249 & 55,343 & 54,475 & 37,500 & 38,000 & 16.9 \\
\hline Washington U.-St. Louis * & 59 & 57,999 & 58,142 & 51,648 & 51,727 & 37,000 & 37,000 & 16.8 \\
\hline$W^{2}$ aterloo ${ }^{\dagger \ddagger}$ & 33 & 65,337 & 68,405 & 67,035 & 70,072 & 39,328 & 41,683 & 18.9 \\
\hline Wayne State & 100 & 52,524 & 53,075 & 46,387 & 47,315 & 40,000 & 40,500 & 16.6 \\
\hline Western Ontario ${ }^{\dagger \ddagger}$ & 62 & 54,221 & 58,941 & 49,869 & 54,246 & 40,958 & 46,000 & 14.6 \\
\hline Wisconsin ${ }^{\star}$ & 149 & 60,691 & 59,685 & 58,640 & 57,766 & 39,338 & 39,338 & 16.7 \\
\hline Yale ${ }^{\dagger+}$ & 216 & 74,753 & 77,131 & 68,987 & 72,263 & 48,100 & 49,500 & 17.8 \\
\hline York ${ }^{\dagger \ddagger}$ & 55 & 73,646 & 81,659 & 69,450 & 76,495 & 36,310 & 43,275 & 16.5 \\
\hline Cancludes medical and la & $\begin{array}{l}\text { ies. See T } \\
\text { J.S. dollar }\end{array}$ & 35 and 4 & statistics $r$ & to medi & d law libr & laries. & 11 salary s & \\
\hline
\end{tabular}


TABLE 8: BEGINNING PROFESSIONAL SALARIES IN ARL UNIVERSITY LIBRARIES RANK ORDER TABLE, FY 2006-2007*

\begin{tabular}{|c|c|c|c|c|c|}
\hline Rank & Institution & Salary & Rank & Institution & Salary \\
\hline 1 & Kent State & $\$ 51,333$ & 58 & Boston College & $\$ 39,850$ \\
\hline 2 & Columbia & 49,500 & 59 & McGill & 39,557 \\
\hline 2 & New York & 49,500 & 60 & Massachusetts & 39,544 \\
\hline 4 & Yale & 48,100 & 61 & Southern Illinois & 39,500 \\
\hline 5 & Connecticut & 48,000 & 62 & Wisconsin & 39,338 \\
\hline 6 & Michigan State & 47,000 & 63 & Waterloo & 39,328 \\
\hline 7 & Johns Hopkins & 46,700 & 64 & Calif. Berkeley & 39,000 \\
\hline 8 & North Carolina State & 46,000 & 64 & Calif. Davis & 39,000 \\
\hline 9 & Chicago & 45,500 & 64 & Calif. Irvine & 39,000 \\
\hline 9 & Harvard & 45,500 & 64 & Calif. Los Angeles & 39,000 \\
\hline 11 & Georgetown & 45,000 & 64 & Calif. Santa Barbara & 39,000 \\
\hline 11 & MIT & 45,000 & 64 & Delaware & 39,000 \\
\hline 11 & Southern California & 45,000 & 64 & Houston & 39,000 \\
\hline 11 & SUNY Buffalo & 45,000 & 71 & Manitoba & 38,833 \\
\hline 15 & Auburn & 44,700 & 72 & SUNY Albany & 38,500 \\
\hline 16 & Rutgers & 44,525 & 73 & Queen's & 38,452 \\
\hline 17 & Brigham Young & 44,000 & 74 & Hawaii & 38,356 \\
\hline 17 & Georgia Tech & 44,000 & 75 & Dartmouth & 38,000 \\
\hline 17 & Texas & 44,000 & 75 & Minnesota & 38,000 \\
\hline 20 & British Columbia & 43,718 & 75 & Temple & 38,000 \\
\hline 21 & Arizona & 43,474 & 75 & Texas Tech & 38,000 \\
\hline 22 & Cornell & 43,300 & 75 & Vanderbilt & 38,000 \\
\hline 23 & Arizona State & 43,000 & 80 & Guelph & 37,574 \\
\hline 23 & Illinois, Urbana & 43,000 & 81 & Kentucky & 37,500 \\
\hline 23 & Purdue & 43,000 & 81 & Washington State & 37,500 \\
\hline 26 & Calif. Riverside & 42,204 & 83 & Notre Dame & 37,250 \\
\hline 27 & Florida & 42,000 & 84 & Alabama & 37,000 \\
\hline 27 & George Washington & 42,000 & 84 & Brown & 37,000 \\
\hline 27 & Illinois, Chicago & 42,000 & 84 & Cincinnati & 37,000 \\
\hline 27 & North Carolina & 42,000 & 84 & Emory & 37,000 \\
\hline 27 & Pennsylvania & 42,000 & 84 & Northwestern & 37,000 \\
\hline 27 & SUNY Stony Brook & 42,000 & 84 & Tulane & 37,000 \\
\hline 27 & Texas A\&M & 42,000 & 84 & Virginia Tech & 37,000 \\
\hline 34 & Toronto & 41,878 & 84 & Washington-St. Louis & 37,000 \\
\hline 35 & Virginia & 41,800 & 92 & Indiana & 36,952 \\
\hline 36 & Miami & 41,700 & 93 & Duke & 36,850 \\
\hline 37 & Colorado & 41,000 & 94 & Laval & 36,704 \\
\hline 37 & Florida State & 41,000 & 95 & Rochester & 36,700 \\
\hline 37 & Iowa State & 41,000 & 96 & McMaster & 36,311 \\
\hline 40 & Western Ontario & 40,958 & 97 & York & 36,310 \\
\hline 41 & Nebraska & 40,500 & 98 & Rice & 36,099 \\
\hline 41 & Ohio State & 40,500 & 99 & Louisiana State & 36,000 \\
\hline 43 & Alberta & 40,449 & 99 & Louisville & 36,000 \\
\hline 44 & Calif. San Diego & 40,008 & 99 & Oklahoma State & 36,000 \\
\hline 45 & Colorado State & 40,000 & 102 & Saskatchewan & 35,951 \\
\hline 45 & Iowa & 40,000 & 103 & Howard & 35,409 \\
\hline 45 & Kansas & 40,000 & 104 & Case Western Reserve & 35,000 \\
\hline 45 & Maryland & 40,000 & 104 & Ohio & 35,000 \\
\hline 45 & Michigan & 40,000 & 104 & Oregon & 35,000 \\
\hline 45 & New Mexico & 40,000 & 107 & Montreal & 34,504 \\
\hline 45 & Oklahoma & 40,000 & 108 & Missouri & 34,000 \\
\hline 45 & Pennsylvania State & 40,000 & 108 & Pittsburgh & 34,000 \\
\hline 45 & Princeton & 40,000 & 108 & South Carolina & 34,000 \\
\hline 45 & Tennessee & 40,000 & 111 & Georgia & 32,500 \\
\hline 45 & Utah & 40,000 & 112 & Boston University & 24,700 \\
\hline 45 & Washington & 40,000 & 113 & Syracuse & $\mathrm{N} / \mathrm{A}$ \\
\hline 45 & Wayne State & 40,000 & & & \\
\hline
\end{tabular}

\footnotetext{
* Reprinted from ARL Annual Salary Survey 2006-07. Beginning salary figures represent officially designated base, not necessarily salaries of actual incumbents. Excludes medical and law libraries. See Tables 36 and 43 for statistics related to medical and law library salaries.

${ }^{\ddagger}$ Canadian salaries expressed in U.S. dollars.
} 
TABLE 9: BEGINNING PROFESSIONAL SALARIES IN ARL UNIVERSITY LIBRARIES RANK ORDER TABLE, FY 2007-2008*

\begin{tabular}{|c|c|c|c|c|c|}
\hline Rank & Institution & Salary & Rank & Institution & Salary \\
\hline 1 & Columbia & 51,500 & 58 & Colorado & 41,000 \\
\hline 2 & New York & 51,000 & 58 & Michigan & 41,000 \\
\hline 3 & Yale & 49,500 & 58 & Southern Illinois & 41,000 \\
\hline 4 & Johns Hopkins & 48,335 & 58 & Washington & 41,000 \\
\hline 5 & Connecticut & 48,000 & 62 & Boston University & 40,800 \\
\hline 5 & MIT & 48,000 & 63 & Massachusetts & 40,730 \\
\hline 7 & Arizona & 47,325 & 64 & Queen's & 40,695 \\
\hline 8 & Harvard & 47,100 & 65 & McGill & 40,625 \\
\hline 9 & Illinois, Chicago & 47,000 & $\begin{array}{l}05 \\
65\end{array}$ & Western Ontario & 40,625 \\
\hline 9 & Michigan State & 47,000 & 67 & Wayne State & 40,500 \\
\hline 9 & North Carolina State & 47,000 & 68 & Laval & 40,025 \\
\hline 12 & Chicago & 46,800 & 69 & Calif. Berkeley & 40,008 \\
\hline 13 & Rutgers & 46,781 & 69 & Calif. Davis & 40,008 \\
\hline 14 & Brigham Young & 46,000 & 69 & Calif. Irvine & 40,008 \\
\hline 15 & British Columbia & 45,784 & 69 & Calif. Los Angeles & 40,008 \\
\hline 16 & Cornell & 45,000 & 69 & Calif. San Diego & 40,008 \\
\hline 16 & Georgetown & 45,000 & 74 & Alabama & 40,000 \\
\hline 16 & Miami & 45,000 & 74 & Dartmouth & 40,000 \\
\hline 16 & Nebraska & 45,000 & 74 & Houston & 40,000 \\
\hline 16 & Princeton & 45,000 & 74 & Indiana & 40,000 \\
\hline 16 & Southern California & 45,000 & 74 & Iowa & 40,000 \\
\hline 16 & SUNY Buffalo & 45,000 & 74 & Kansas & 40,000 \\
\hline 16 & Texas & 45,000 & 74 & Minnesota & 40,000 \\
\hline 16 & Texas Tech & 45,000 & 74 & New Mexico & 40,000 \\
\hline 25 & Auburn & 44,720 & 74 & Notre Dame & 40,000 \\
\hline 26 & Illinois, Urbana & 44,500 & 74 & Oklahoma & 40,000 \\
\hline 27 & Texas A\&M & 44,000 & 84 & Wisconsin & 39,338 \\
\hline 28 & Delaware & 43,600 & 85 & Rice & 39,060 \\
\hline 29 & Colorado State & 43,500 & 86 & Northwestern & 39,000 \\
\hline 29 & Purdue & 43,500 & 86 & Temple & 39,000 \\
\hline 31 & Kent State & 43,389 & 86 & Vanderbilt & 39,000 \\
\hline 32 & York & 43,275 & 89 & McMaster & 38,598 \\
\hline 33 & Toronto & 43,010 & 90 & Guelph & 38,590 \\
\hline 34 & Arizona State & 43,000 & 91 & Kentucky & 38,500 \\
\hline 34 & Duke & 43,000 & 91 & Missouri & 38,500 \\
\hline 34 & SUNY Stony Brook & 43,000 & 91 & Virginia Tech & 38,500 \\
\hline 34 & Virginia & 43,000 & 94 & Brown & 38,000 \\
\hline 38 & Alberta & 42,997 & 94 & Cincinnati & 38,000 \\
\hline 39 & Iowa State & 42,500 & 94 & Emory & 38,000 \\
\hline 39 & Pennsylvania & 42,500 & 94 & Louisiana State & 38,000 \\
\hline 41 & Calif. Riverside & 42,204 & 94 & SUNY Albany & 38,000 \\
\hline 42 & Hawaii & 42,026 & $\begin{array}{l}94 \\
94\end{array}$ & Washington State & 38,000 \\
\hline 43 & Calif. Santa Barbara & 42,008 & 100 & Louisville & 37,000 \\
\hline 44 & Florida & 42,000 & 100 & Tulane & 37,000 \\
\hline 44 & Florida State & 42,000 & 100 & Washington-St. Louis & 37,000 \\
\hline 44 & George Washington & 42,000 & 103 & Rochester & 36,700 \\
\hline 44 & Georgia Tech & 42,000 & 104 & Montreal & 36,144 \\
\hline 44 & Maryland & 42,000 & 105 & Ohio & 36,000 \\
\hline 44 & North Carolina & 42,000 & 105 & Oklahoma State & 36,000 \\
\hline 44 & Tennessee & 42,000 & 107 & Howard & 35,049 \\
\hline 44 & Utah & 42,000 & 108 & Case Western Reserve & 35,000 \\
\hline 52 & Waterloo & 41,683 & $\begin{array}{l}108 \\
108\end{array}$ & Georgia & 35,000 \\
\hline 53 & Saskatchewan & 41,598 & $\begin{array}{l}100 \\
108\end{array}$ & Oregon & 35,000 \\
\hline 54 & Ohio State & 41,500 & 111 & Pittsburgh & 34,000 \\
\hline 55 & Manitoba & 41,331 & 111 & South Carolina & 34,000 \\
\hline 56 & Pennsylvania State & 41,200 & 113 & Syracuse & N/A \\
\hline 57 & Boston College & 41,050 & & & \\
\hline
\end{tabular}

\footnotetext{
* Beginning salary figures represent officially designated base, not necessarily salaries of actual incumbents. Excludes medical and law libraries. See Tables 36 and 43 for statistics related to medical and law library salaries.

† See Footnotes.

${ }^{\ddagger}$ Canadian salaries expressed in U.S. dollars.
} 
TABLE 10: MEDIAN PROFESSIONAL SALARIES IN ARL UNIVERSITY LIBRARIES RANK ORDER TABLE, FY 2006-2007*

\begin{tabular}{|c|c|c|c|c|c|}
\hline Rank & Institution & Salary & Rank & Institution & Salary \\
\hline 1 & Rutgers & $\$ 80,048$ & 58 & Wisconsin & $\$ 58,640$ \\
\hline 2 & Toronto & 77,290 & 59 & Brigham Young & 58,500 \\
\hline 3 & Alberta & 76,714 & 60 & Tulane & 58,294 \\
\hline 4 & Manitoba & 74,814 & 61 & Brown & 58,193 \\
\hline 5 & California, Irvine & 73,537 & 62 & George Washington & 57,907 \\
\hline 6 & California, Berkeley & 72,558 & 63 & Colorado & 57,622 \\
\hline 7 & California, Los Angeles & 72,084 & 64 & Indiana & 57,123 \\
\hline 8 & California, Davis & 71,916 & 65 & Miami & 56,863 \\
\hline 9 & New Mexico & 71,319 & 66 & Arizona State & 56,743 \\
\hline 10 & California, San Diego & 70,350 & 67 & Texas & 56,619 \\
\hline 11 & Massachusetts & 69,658 & 68 & SUNY Albany & 56,376 \\
\hline 12 & York & 69,450 & 69 & Pittsburgh & 56,345 \\
\hline 13 & Yale & 68,987 & 70 & Virginia Tech & 56,150 \\
\hline 14 & Queen`s & 68,700 & 71 & Illinois, Urbana & 56,091 \\
\hline 15 & Connecticut & 67,825 & 72 & Iowa State & 56,054 \\
\hline 16 & Waterloo & 67,035 & 73 & Laval & 55,855 \\
\hline 17 & McGill & 66,226 & 74 & Syracuse & 55,757 \\
\hline 18 & Chicago & 65,792 & 75 & Washington & 55,656 \\
\hline 19 & SUNY Stony Brook & 65,764 & 76 & Duke & 55,400 \\
\hline 20 & Dartmouth & 65,563 & 77 & Washington State & 55,343 \\
\hline 21 & California, Santa Barbara & 65,448 & 78 & Kansas & 54,982 \\
\hline 22 & California, Riverside & 65,220 & 79 & Ohio State & 54,834 \\
\hline 23 & Princeton & 64,600 & 80 & Montreal & 54,360 \\
\hline 24 & Harvard & 64,443 & 81 & Kentucky & 54,352 \\
\hline 25 & Saskatchewan & 64,350 & 82 & Purdue & 54,330 \\
\hline 26 & Columbia & 64,160 & 83 & Iowa & 54,207 \\
\hline 27 & Louisville & 64,134 & 84 & SUNY Buffalo & 53,790 \\
\hline 28 & Delaware & 64,130 & 85 & Georgia Tech & 53,732 \\
\hline 29 & MIT & 64,100 & 86 & Illinois, Chicago & 52,981 \\
\hline 30 & Notre Dame & 63,766 & 87 & Nebraska & 52,928 \\
\hline 31 & New York University & 63,700 & 88 & Case Western Reserve & 52,842 \\
\hline 32 & British Columbia & 62,938 & 89 & Houston & 52,799 \\
\hline 33 & Southern California & 62,900 & 90 & Washington U.-St. Louis & 51,648 \\
\hline 34 & Tennessee & 62,319 & 91 & Utah & 51,633 \\
\hline 35 & Virginia & 61,800 & 92 & Rice & 51,600 \\
\hline 36 & Boston College & 61,750 & 93 & Florida & 51,413 \\
\hline 37 & Pennsylvania State & 61,308 & 94 & Kent State & 51,333 \\
\hline 38 & McMaster & 61,044 & 95 & Vanderbilt & 51,264 \\
\hline 39 & Minnesota & 61,033 & 96 & Texas A\&M & 50,502 \\
\hline 40 & Hawaii & 60,959 & 97 & Boston University & 50,000 \\
\hline 41 & Colorado State & 60,828 & 98 & Western Ontario & 49,869 \\
\hline 42 & Northwestern & 60,144 & 99 & Rochester & 49,733 \\
\hline 43 & Cornell & 60,050 & 100 & Oklahoma State & 49,536 \\
\hline 44 & North Carolina & 60,000 & 101 & Alabama & 49,428 \\
\hline 45 & Michigan & 59,920 & 102 & Missouri & 49,134 \\
\hline 46 & Johns Hopkins & 59,795 & 103 & Oregon & 48,424 \\
\hline 47 & Michigan State & 59,700 & 104 & Georgia & 47,731 \\
\hline 48 & Pennsylvania & 59,665 & 105 & Texas Tech & 47,410 \\
\hline 49 & North Carolina State & 59,500 & 106 & South Carolina & 47,388 \\
\hline 50 & Maryland & 59,395 & 107 & Southern Illinois & 47,311 \\
\hline 51 & Georgetown & 59,375 & 108 & Howard & 47,105 \\
\hline 52 & Auburn & 59,200 & 109 & Wayne State & 46,387 \\
\hline 53 & Cincinnati & 58,987 & 110 & Oklahoma & 46,162 \\
\hline 54 & Emory & 58,925 & 111 & Ohio University & 44,263 \\
\hline 55 & Arizona & 58,917 & 112 & Louisiana State & 43,705 \\
\hline 56 & Guelph & 58,833 & 113 & Florida State & 42,479 \\
\hline 57 & Temple & 58,670 & & & \\
\hline
\end{tabular}

* Reprinted from ARL Annual Salary Survey 2006-07. Salaries of directors are not included in the calculation of medians. Excludes medical and law libraries. See Tables 37 and 44 for statistics related to medical and law library salaries.

$\uparrow$ See Footnotes.

‡Canadian salaries expressed in U.S. dollars. 
TABLE 11: MEDIAN PROFESSIONAL SALARIES IN ARL UNIVERSITY LIBRARIES RANK ORDER TABLE, FY 2007-2008*

\begin{tabular}{|c|c|c|c|c|c|}
\hline Rank & Institution & Salary & Rank & Institution & Salary \\
\hline 1 & Rutgers & $\$ 84,829$ & 58 & Miami & $\$ 60,000$ \\
\hline 2 & Alberta & 82,536 & 58 & Virginia & 60,000 \\
\hline 3 & California, Davis & 79,968 & 60 & Indiana & 59,705 \\
\hline 4 & Manitoba & 79,159 & 61 & Virginia Tech & 59,672 \\
\hline 5 & California, Berkeley & 79,108 & 62 & George Washington & 59,401 \\
\hline 6 & Toronto & 78,443 & 63 & Auburn & 58,540 \\
\hline 7 & York & 76,495 & 64 & Pennsylvania & 58,526 \\
\hline 8 & New Mexico & 74,768 & 65 & Pittsburgh & 58,451 \\
\hline 9 & Connecticut & 74,661 & 66 & Guelph & 58,271 \\
\hline 10 & Saskatchewan & 74,456 & 67 & Syracuse & 58,263 \\
\hline 11 & Queen`s & 74,078 & 68 & Illinois, Urbana & 58,247 \\
\hline 12 & California ,Los Angeles & 73,382 & 69 & Montreal & 58,216 \\
\hline 13 & California, Irvine & 73,356 & 70 & Washington & 58,020 \\
\hline 13 & California, San Diego & 73,356 & 71 & Kentucky & 57,817 \\
\hline 15 & Yale & 72,263 & 72 & Purdue & 57,815 \\
\hline 16 & Dartmouth & 71,597 & 73 & Texas & 57,809 \\
\hline 17 & Waterloo & 70,072 & 74 & Wisconsin & 57,766 \\
\hline 18 & Princeton & 70,000 & 75 & Iowa State & 57,452 \\
\hline 19 & Massachusetts & 69,746 & 76 & Michigan State & 57,000 \\
\hline 20 & McMaster & 69,173 & 77 & SUNY Albany & 56,625 \\
\hline 21 & New York University & 68,882 & 78 & Temple & 56,572 \\
\hline 22 & British Columbia & 68,737 & 79 & Iowa & 55,700 \\
\hline 23 & Chicago & 68,418 & 80 & Tulane & 55,664 \\
\hline 24 & California, Riverside & 68,000 & 81 & Duke & 55,650 \\
\hline 25 & SUNY Stony Brook & 67,828 & 82 & Kansas & 55,120 \\
\hline 26 & Harvard & 67,486 & 83 & Rice & 54,900 \\
\hline 27 & California, Santa Barbara & 66,756 & 84 & Illinois, Chicago & 54,885 \\
\hline 28 & Notre Dame & 65,769 & 85 & Texas A\&M & 54,857 \\
\hline 29 & MIT & 65,650 & 86 & Georgia Tech & 54,631 \\
\hline 30 & Tennessee & 65,394 & 87 & Nebraska & 54,624 \\
\hline 31 & Boston College & 65,293 & 88 & Utah & 54,614 \\
\hline 32 & McGill & 64,728 & 89 & Washington State & 54,475 \\
\hline 33 & Southern California & 64,335 & 90 & Case Western Reserve & 54,335 \\
\hline 34 & Delaware & 64,235 & 91 & Western Ontario & 54,246 \\
\hline 35 & Minnesota & 63,532 & 92 & Kent State & 53,777 \\
\hline 36 & Michigan & 63,413 & 93 & Howard & 53,359 \\
\hline 37 & North Carolina & 62,825 & 94 & Texas Tech & 53,079 \\
\hline 38 & Pennsylvania State & 62,730 & 95 & Ohio State & 52,941 \\
\hline 39 & Laval & 62,715 & 96 & Alabama & 52,891 \\
\hline 40 & Cornell & 62,490 & 97 & Vanderbilt & 52,479 \\
\hline 41 & Arizona State & 62,333 & 98 & Florida & 52,327 \\
\hline 42 & Brown & 61,998 & 99 & Washington U.-St .Louis & 51,727 \\
\hline 43 & Louisville & 61,986 & 100 & Boston University & 51,450 \\
\hline 44 & Maryland & 61,930 & 101 & Rochester & 51,282 \\
\hline 45 & North Carolina State & 61,717 & 102 & Oregon & 51,176 \\
\hline 46 & Columbia & 61,585 & 103 & Oklahoma & 50,823 \\
\hline 47 & Cincinnati & 61,552 & 104 & Missouri & 50,442 \\
\hline 48 & Georgetown & 61,508 & 105 & Oklahoma State & 49,824 \\
\hline 49 & Colorado & 61,431 & 106 & Georgia & 49,634 \\
\hline 50 & Northwestern & 61,203 & 107 & Southern Illinois & 49,375 \\
\hline 51 & Emory & 61,000 & 108 & Houston & 49,037 \\
\hline 52 & SUNY Buffalo & 60,852 & 109 & South Carolina & 47,983 \\
\hline 53 & Colorado State & 60,650 & 110 & Wayne State & 47,315 \\
\hline 54 & Hawaii & 60,354 & 111 & Florida State & 45,752 \\
\hline 55 & Brigham Young & 60,200 & 112 & Ohio University & 45,010 \\
\hline 56 & Arizona & 60,136 & 113 & Louisiana State & 44,879 \\
\hline 57 & Johns Hopkins & 60,060 & & & \\
\hline
\end{tabular}

* Salaries of directors are not included in the calculation of medians. Excludes medical and law libraries. See Tables 37 and 44 for statistics related to medical and law library salaries.

† Canadian salaries expressed in U.S. dollars. 
TABLE 12: AVERAGE PROFESSIONAL SALARIES IN ARL UNIVERSITY LIBRARIES RANK ORDER TABLE, FY 2006-2007*

\begin{tabular}{|c|c|c|c|c|c|}
\hline Rank & Institution & Salary & Rank & Institution & Salary \\
\hline 1 & Rutgers & $\$ 77,298$ & 58 & Syracuse & $\$ 62,119$ \\
\hline 2 & California, Berkeley & 75,968 & 59 & Michigan State & 62,101 \\
\hline 3 & Yale & 74,753 & 60 & Emory & 61,950 \\
\hline 4 & Toronto & 74,374 & 61 & Maryland & 61,723 \\
\hline 5 & California, Los Angeles & 74,082 & 62 & Pittsburgh & 61,695 \\
\hline 6 & York & 73,646 & 63 & Purdue & 61,429 \\
\hline 7 & Connecticut & 72,674 & 64 & McMaster & 61,406 \\
\hline 8 & California, Irvine & 72,485 & 65 & Indiana & 60,776 \\
\hline 9 & Manitoba & 72,277 & 66 & Wisconsin & 60,691 \\
\hline 10 & New York University & 72,220 & 67 & Iowa & 60,666 \\
\hline 11 & California, San Diego & 71,287 & 68 & Brigham Young & 60,562 \\
\hline 12 & Columbia & 70,854 & 69 & Arizona State & 60,315 \\
\hline 13 & New Mexico & 70,749 & 70 & Washington & 60,054 \\
\hline 14 & Chicago & 70,385 & 71 & Colorado & 59,930 \\
\hline 15 & Harvard & 70,160 & 72 & Georgia Tech & 59,700 \\
\hline 16 & Princeton & 70,082 & 73 & Tulane & 59,287 \\
\hline 17 & Alberta & 69,228 & 74 & Virginia Tech & 59,256 \\
\hline 18 & California, Davis & 69,196 & 75 & Duke & 58,918 \\
\hline 19 & California, Santa Barbara & 68,946 & 76 & Iowa State & 58,887 \\
\hline 20 & Dartmouth & 68,775 & 77 & Auburn & 58,800 \\
\hline 21 & Southern California & 68,457 & 78 & Nebraska & 58,792 \\
\hline 22 & SUNY Stony Brook & 68,185 & 79 & Washington State & 58,761 \\
\hline 23 & Queen`s & 67,488 & 80 & SUNY Albany & 58,663 \\
\hline 24 & MIT & 67,285 & 81 & SUNY Buffalo & 58,519 \\
\hline 25 & Massachusetts & 67,202 & 82 & Rice & 58,302 \\
\hline 26 & Delaware & 66,598 & 83 & Illinois, Chicago & 58,010 \\
\hline 27 & North Carolina State & 66,529 & 84 & Washington U.-St. Louis & 57,999 \\
\hline 28 & Cornell & 66,479 & 85 & Ohio State & 57,544 \\
\hline 29 & California, Riverside & 66,183 & 86 & Kansas & 57,251 \\
\hline 30 & Waterloo & 65,337 & 87 & Montreal & 56,909 \\
\hline 31 & Saskatchewan & 64,936 & 88 & Houston & 56,901 \\
\hline 32 & Notre Dame & 64,821 & 89 & Vanderbilt & 56,839 \\
\hline 33 & Virginia & 64,777 & 90 & Utah & 56,452 \\
\hline 34 & Pennsylvania State & 64,546 & 91 & Kentucky & 56,371 \\
\hline 35 & Michigan & 64,295 & 92 & Texas A\&M & 56,327 \\
\hline 36 & Boston College & 64,187 & 93 & Florida & 55,761 \\
\hline 37 & Minnesota & 64,164 & 94 & Case Western Reserve & 54,258 \\
\hline 38 & Arizona & 63,916 & 95 & Western Ontario & 54,221 \\
\hline 39 & McGill & 63,903 & 96 & Kent State & 54,156 \\
\hline 40 & George Washington & 63,878 & 97 & Laval & 54,114 \\
\hline 41 & British Columbia & 63,845 & 98 & Alabama & 54,096 \\
\hline 42 & Miami & 63,695 & 99 & Georgia & 54,062 \\
\hline 43 & Georgetown & 63,686 & 100 & Southern Illinois & 53,791 \\
\hline 44 & Johns Hopkins & 63,555 & 101 & Oklahoma State & 53,406 \\
\hline 45 & Tennessee & 63,432 & 102 & Rochester & 53,255 \\
\hline 46 & Temple & 63,282 & 103 & Wayne State & 52,524 \\
\hline 47 & North Carolina & 63,189 & 104 & Boston University & 52,505 \\
\hline 48 & Louisville & 63,179 & 105 & Missouri & 52,090 \\
\hline 49 & Cincinnati & 62,957 & 106 & Texas Tech & 51,858 \\
\hline 50 & Northwestern & 62,941 & 107 & Oregon & 51,535 \\
\hline 51 & Hawaii & 62,760 & 108 & South Carolina & 51,060 \\
\hline 52 & Pennsylvania & 62,553 & 109 & Howard & 50,032 \\
\hline 53 & Brown & 62,460 & 110 & Oklahoma & 49,386 \\
\hline 54 & Illinois, Urbana & 62,401 & 111 & Ohio University & 48,815 \\
\hline 55 & Colorado State & 62,371 & 112 & Louisiana State & 47,812 \\
\hline 56 & Guelph & 62,335 & 113 & Florida State & 46,853 \\
\hline 57 & Texas & 62,185 & & & \\
\hline
\end{tabular}

* Reprinted from ARL Annual Salary Survey 2006-07. Salaries of directors are not included in the calculation of averages. Excludes medical and law libraries. See Tables 38 and 45 for statistics related to medical and law library salaries.

† See Footnotes.

‡ Canadian salaries expressed in U.S. dollars. 
TABLE 13: AVERAGE PROFESSIONAL SALARIES IN ARL UNIVERSITY LIBRARIES RANK ORDER TABLE, FY 2007-2008*

\begin{tabular}{|c|c|c|c|c|c|}
\hline Rank & Institution & Salary & Rank & Institution & Salary \\
\hline 1 & Rutgers & $\$ 82,023$ & 58 & SUNY Buffalo & $\$ 64,414$ \\
\hline 2 & York & 81,659 & 59 & Arizona State & 64,325 \\
\hline 3 & California, Berkeley & 80,749 & 60 & Indiana & 64,257 \\
\hline 4 & New York University & 77,595 & 61 & Pittsburgh & 64,047 \\
\hline 5 & Manitoba & 77,261 & 62 & Illinois, Urbana & 64,009 \\
\hline 6 & Yale & 77,131 & 63 & Washington & 63,655 \\
\hline 7 & Connecticut & 76,946 & 64 & Texas & 63,467 \\
\hline 8 & Toronto & 76,710 & 65 & Iowa & 63,234 \\
\hline 9 & New Mexico & 76,612 & 66 & Pennsylvania & 63,029 \\
\hline 10 & California, Los Angeles & 76,029 & 67 & Virginia Tech & 62,691 \\
\hline 11 & Alberta & 74,787 & 68 & Colorado State & 62,333 \\
\hline 12 & Saskatchewan & 74,526 & 69 & Guelph & 62,069 \\
\hline 13 & Princeton & 74,179 & 70 & Brigham Young & 61,929 \\
\hline 14 & California, San Diego & 74,176 & 71 & Michigan State & 61,743 \\
\hline 15 & California, Davis & 73,853 & 72 & Rice & 61,662 \\
\hline 16 & Harvard & 72,861 & 73 & Nebraska & 61,376 \\
\hline 17 & Queen`s & 72,268 & 74 & Hawaii & 61,330 \\
\hline 18 & Chicago & 72,179 & 75 & Auburn & 61,104 \\
\hline 19 & Dartmouth & 71,642 & 76 & Duke & 60,715 \\
\hline 20 & California, Irvine & 71,572 & 77 & SUNY Albany & 60,304 \\
\hline 21 & Columbia & 70,838 & 78 & Montreal & 60,262 \\
\hline 22 & Southern California & 70,463 & 79 & Texas A\&M & 60,024 \\
\hline 23 & California, Santa Barbara & 70,227 & 80 & Utah & 59,993 \\
\hline 24 & SUNY Stony Brook & 70,027 & 81 & Texas Tech & 59,987 \\
\hline 25 & MIT & 69,719 & 82 & Iowa State & 59,891 \\
\hline 26 & British Columbia & 69,414 & 83 & Wisconsin & 59,685 \\
\hline 27 & Massachusetts & 69,319 & 84 & Laval & 59,630 \\
\hline 28 & California, Riverside & 69,120 & 85 & Kentucky & 59,613 \\
\hline 29 & Delaware & 69,033 & 86 & Kansas & 59,070 \\
\hline 30 & North Carolina State & 68,461 & 87 & Tulane & 59,023 \\
\hline 31 & Waterloo & 68,405 & 88 & Western Ontario & 58,941 \\
\hline 32 & Tennessee & 68,333 & 89 & Illinois, Chicago & 58,925 \\
\hline 33 & Cornell & 68,228 & 90 & Georgia Tech & 58,753 \\
\hline 34 & North Carolina & 67,938 & 91 & Washington State & 58,249 \\
\hline 35 & Boston College & 67,847 & 92 & Alabama & 58,232 \\
\hline 36 & Virginia & 67,602 & 93 & Washington U.-St. Louis & 58,142 \\
\hline 37 & McMaster & 67,549 & 94 & Vanderbilt & 57,775 \\
\hline 38 & Notre Dame & 66,827 & 95 & Missouri & 57,607 \\
\hline 39 & Pennsylvania State & 66,725 & 96 & Case Western Reserve & 57,089 \\
\hline 40 & Georgetown & 66,689 & 97 & Georgia & 57,082 \\
\hline 41 & Michigan & 66,608 & 98 & Ohio State & 56,745 \\
\hline 42 & Minnesota & 66,067 & 99 & Houston & 56,608 \\
\hline 43 & McGill & 66,010 & 100 & Florida & 56,403 \\
\hline 44 & Johns Hopkins & 65,765 & 101 & Kent State & 56,086 \\
\hline 45 & Temple & 65,552 & 102 & Rochester & 55,411 \\
\hline 46 & Colorado & 65,510 & 103 & Oregon & 54,999 \\
\hline 47 & Brown & 65,442 & 104 & Oklahoma State & 54,593 \\
\hline 48 & Purdue & 65,312 & 105 & Southern Illinois & 54,562 \\
\hline 49 & Cincinnati & 65,283 & 106 & Boston University & 54,472 \\
\hline 50 & Northwestern & 65,234 & 107 & Oklahoma & 53,241 \\
\hline 51 & Louisville & 65,230 & 108 & Howard & 53,211 \\
\hline 52 & Miami & 65,171 & 109 & Wayne State & 53,075 \\
\hline 53 & Syracuse & 65,131 & 110 & South Carolina & 51,457 \\
\hline 54 & George Washington & 64,950 & 111 & Ohio University & 48,853 \\
\hline 55 & Maryland & 64,926 & 112 & Louisiana State & 48,711 \\
\hline 56 & Arizona & 64,731 & 113 & Florida State & 48,656 \\
\hline 57 & Emory & 64,551 & & & \\
\hline
\end{tabular}

* Salaries of directors are not included in the calculation of averages. Excludes medical and law libraries. See Tables 38 and 45 for statistics related to medical and law library salaries.

$\dagger$ Canadian salaries expressed in U.S. dollars. 


\section{TABLE 14: AVERAGE, MEDIAN, AND BEGINNING PROFESSIONAL SALARIES IN ARL UNIVERSITY LIBRARIES \\ SUMMARY OF RANKINGS, FYs 2004-2005 TO 2007-2008*}

\begin{tabular}{|c|c|c|c|c|c|c|c|c|c|c|c|c|}
\hline \multirow{2}{*}{$\begin{array}{l}\text { Institution } \\
\end{array}$} & \multicolumn{4}{|c|}{ Average Salaries } & \multicolumn{4}{|c|}{ Median Salaries } & \multicolumn{4}{|c|}{ Beginning Salaries } \\
\hline & 2005 & 2006 & 2007 & 2008 & 2005 & 2006 & 2007 & 2008 & 2005 & 2006 & 2007 & 2008 \\
\hline Alabama & 108 & 97 & 98 & 92 & 109 & 100 & 101 & 96 & 73 & 92 & 84 & 74 \\
\hline Alberta & 44 & 28 & 17 & 11 & 18 & 6 & 3 & 2 & 98 & 77 & 43 & 38 \\
\hline Arizona & 40 & 40 & 38 & 56 & 70 & 77 & 55 & 56 & 8 & 22 & 21 & 7 \\
\hline Arizona State & 85 & 84 & 69 & 59 & 76 & 69 & 66 & 41 & 36 & 51 & 23 & 34 \\
\hline Auburn & 82 & 78 & 77 & 75 & 80 & 66 & 52 & 63 & 16 & 15 & 15 & 25 \\
\hline Boston University & 99 & 95 & 104 & 106 & 98 & 98 & 97 & 100 & 99 & 107 & 112 & 62 \\
\hline Boston College & 29 & 31 & 36 & 35 & 35 & 35 & 36 & 31 & 51 & 50 & 58 & 57 \\
\hline Brigham Young & 54 & 54 & 68 & 70 & 43 & 47 & 59 & 55 & 11 & 13 & 17 & 14 \\
\hline British Columbia & 73 & 74 & 41 & 26 & 57 & 67 & 32 & 22 & 94 & 88 & 20 & 15 \\
\hline Brown & 30 & 36 & 53 & 47 & 29 & 37 & 61 & 42 & 83 & 89 & 84 & 94 \\
\hline California, Berkeley & 1 & 1 & 2 & 3 & 2 & 1 & 6 & 5 & 44 & 57 & 64 & 69 \\
\hline California, Davis & 6 & 7 & 18 & 15 & 2 & 3 & 8 & 3 & 44 & 57 & 64 & 69 \\
\hline California, Irvine & 8 & 13 & 8 & 20 & 5 & 3 & 5 & 13 & 44 & 57 & 64 & 69 \\
\hline California, Los Angeles & 2 & 3 & 5 & 10 & 1 & 3 & 7 & 12 & 44 & 57 & 64 & 69 \\
\hline California, Riverside & 20 & 25 & 29 & 28 & 10 & 24 & 22 & 24 & 44 & 25 & 26 & 41 \\
\hline California, San Diego & 10 & 11 & 11 & 14 & 9 & 13 & 10 & 13 & 44 & 57 & 44 & 69 \\
\hline California, Santa Barbara & 9 & 14 & 19 & 23 & 6 & 15 & 21 & 27 & 44 & 57 & 64 & 43 \\
\hline Case Western Reserve & 84 & 87 & 94 & 96 & 86 & 79 & 88 & 90 & 73 & 92 & 104 & 108 \\
\hline Chicago & 12 & 15 & 14 & 18 & 19 & 17 & 18 & 23 & 10 & 10 & 9 & 12 \\
\hline Cincinnati & 43 & 41 & 49 & 49 & 45 & 41 & 53 & 47 & 95 & 92 & 84 & 94 \\
\hline Colorado & 57 & 63 & 71 & 46 & 44 & 50 & 63 & 49 & 36 & 28 & 37 & 58 \\
\hline Colorado State & 37 & 53 & 55 & 68 & 31 & 53 & 41 & 53 & 29 & 44 & 45 & 29 \\
\hline Columbia & 14 & 17 & 12 & 21 & 27 & 31 & 26 & 46 & 2 & 3 & 2 & 1 \\
\hline Connecticut & 7 & 6 & 7 & 7 & 14 & 8 & 15 & 9 & 3 & 2 & 5 & 5 \\
\hline Cornell & 25 & 23 & 28 & 33 & 48 & 43 & 43 & 40 & 17 & 23 & 22 & 16 \\
\hline Dartmouth & 19 & 18 & 20 & 19 & 12 & 16 & 20 & 16 & 113 & 68 & 75 & 74 \\
\hline Delaware & 22 & 19 & 26 & 29 & 20 & 18 & 28 & 34 & 29 & 44 & 64 & 28 \\
\hline Duke & 46 & 68 & 75 & 76 & 53 & 74 & 76 & 81 & 58 & 73 & 93 & 34 \\
\hline Emory & 38 & 46 & 60 & 57 & 37 & 46 & 54 & 51 & 73 & 68 & 84 & 94 \\
\hline Florida & 101 & 86 & 93 & 100 & 103 & 91 & 93 & 98 & 17 & 16 & 27 & 44 \\
\hline Florida State & 110 & 109 & 113 & 113 & 112 & 109 & 113 & 111 & 63 & 51 & 37 & 44 \\
\hline George Washington & 24 & 35 & 40 & 54 & 26 & 34 & 62 & 62 & 36 & 16 & 27 & 44 \\
\hline Georgetown & 26 & 32 & 43 & 40 & 34 & 40 & 51 & 48 & 17 & 16 & 11 & 16 \\
\hline Georgia & 95 & 96 & 99 & 97 & 96 & 101 & 104 & 106 & 99 & 112 & 111 & 108 \\
\hline Georgia Tech & 69 & 77 & 72 & 90 & 47 & 86 & 85 & 86 & 17 & 8 & 17 & 44 \\
\hline Guelph & 97 & 76 & 56 & 69 & 90 & 58 & 56 & 66 & 107 & 103 & 80 & 90 \\
\hline Harvard & 18 & 16 & 15 & 16 & 21 & 22 & 24 & 26 & 12 & 11 & 9 & 8 \\
\hline Hawaii & 56 & 52 & 51 & 74 & 39 & 39 & 40 & 54 & 59 & 74 & 74 & 42 \\
\hline Houston & 102 & 112 & 88 & 99 & 106 & 112 & 89 & 108 & 63 & 68 & 64 & 74 \\
\hline Howard & 103 & 102 & 109 & 108 & 104 & 97 & 108 & 93 & 72 & 91 & 103 & 107 \\
\hline Illinois, Chicago & 53 & 73 & 83 & 89 & 75 & 85 & 86 & 84 & 63 & 16 & 27 & 9 \\
\hline Illinois, Urbana & 45 & 42 & 54 & 62 & 69 & 75 & 71 & 68 & 13 & 16 & 23 & 26 \\
\hline
\end{tabular}




\section{TABLE 14: AVERAGE, MEDIAN, AND BEGINNING PROFESSIONAL SALARIES IN ARL UNIVERSITY LIBRARIES \\ SUMMARY OF RANKINGS, FYs 2004-2005 TO 2007-2008*}

\begin{tabular}{|c|c|c|c|c|c|c|c|c|c|c|c|c|}
\hline Institution & \multicolumn{4}{|c|}{ Average Salaries } & \multicolumn{4}{|c|}{ Median Salaries } & \multicolumn{4}{|c|}{ Beginning Salaries } \\
\hline FY & 2005 & 2006 & 2007 & 2008 & 2005 & 2006 & 2007 & 2008 & 2005 & 2006 & 2007 & 2008 \\
\hline Indiana & 31 & 55 & 65 & 60 & 33 & 54 & 64 & 60 & 71 & 78 & 92 & 74 \\
\hline Iowa & 77 & 66 & 67 & 65 & 78 & 81 & 83 & 79 & 73 & 28 & 45 & 74 \\
\hline Iowa State & 78 & 83 & 76 & 82 & 62 & 71 & 72 & 75 & 36 & 28 & 37 & 39 \\
\hline Johns Hopkins & 34 & 39 & 44 & 44 & 48 & 48 & 46 & 57 & 1 & 12 & 7 & 4 \\
\hline Kansas & 62 & 58 & 86 & 86 & 71 & 65 & 78 & 82 & 73 & 28 & 45 & 74 \\
\hline Kent State & 80 & 105 & 96 & 101 & 74 & 94 & 94 & 92 & 9 & 1 & 1 & 31 \\
\hline Kentucky & 96 & 93 & 91 & 85 & 95 & 83 & 81 & 71 & 63 & 64 & 81 & 91 \\
\hline Laval & 105 & 108 & 97 & 84 & 73 & 73 & 73 & 39 & 102 & 98 & 94 & 68 \\
\hline Louisiana State & 111 & 113 & 112 & 112 & 111 & 113 & 112 & 113 & 73 & 87 & 99 & 94 \\
\hline Louisville & 55 & 45 & 48 & 51 & 38 & 26 & 27 & 43 & 73 & 79 & 99 & 100 \\
\hline McGill & 90 & 79 & 39 & 43 & 50 & 36 & 17 & 32 & 108 & 109 & 59 & 65 \\
\hline McMaster & 92 & 85 & 64 & 37 & 81 & 60 & 38 & 20 & 112 & 110 & 96 & 89 \\
\hline Manitoba & 58 & 24 & 9 & 5 & 22 & 10 & 4 & 4 & 103 & 90 & 71 & 55 \\
\hline Maryland & 51 & 60 & 61 & 55 & 46 & 56 & 50 & 44 & 17 & 28 & 45 & 44 \\
\hline Massachusetts & 15 & 20 & 25 & 27 & 7 & 11 & 11 & 19 & 54 & 66 & 60 & 63 \\
\hline MIT & 21 & 21 & 24 & 25 & 28 & 21 & 29 & 29 & 7 & 8 & 11 & 5 \\
\hline Miami & 67 & 56 & 42 & 52 & 61 & 63 & 65 & 58 & 63 & 51 & 36 & 16 \\
\hline Michigan & 39 & 26 & 35 & 41 & 60 & 45 & 45 & 36 & 73 & 28 & 45 & 58 \\
\hline Michigan State & 33 & 49 & 59 & 71 & 35 & 42 & 47 & 76 & 14 & 14 & 6 & 9 \\
\hline Minnesota & 65 & 29 & 37 & 42 & 59 & 30 & 39 & 35 & 63 & 79 & 75 & 74 \\
\hline Missouri & 91 & 98 & 105 & 95 & 100 & 102 & 102 & 104 & 99 & 111 & 108 & 91 \\
\hline Montreal & 107 & 103 & 87 & 78 & 97 & 90 & 80 & 69 & 111 & 106 & 107 & 104 \\
\hline Nebraska & 75 & 82 & 78 & 73 & 83 & 95 & 87 & 87 & 17 & 26 & 41 & 16 \\
\hline New Mexico & 16 & 12 & 13 & 9 & 8 & 9 & 9 & 8 & 27 & 28 & 45 & 74 \\
\hline New York & 13 & 8 & 10 & 4 & 23 & 23 & 31 & 21 & 3 & 4 & 2 & 2 \\
\hline North Carolina & 63 & 50 & 47 & 34 & 66 & 57 & 44 & 37 & 52 & 28 & 27 & 44 \\
\hline North Carolina State & 52 & 48 & 27 & 30 & 64 & 76 & 49 & 45 & 17 & 5 & 8 & 9 \\
\hline Northwestern & 35 & 37 & 50 & 50 & 32 & 38 & 42 & 50 & 85 & 79 & 84 & 86 \\
\hline Notre Dame & 28 & 34 & 32 & 38 & 25 & 33 & 30 & 28 & 63 & 79 & 83 & 74 \\
\hline Ohio University & 104 & 110 & 111 & 111 & 107 & 111 & 111 & 112 & 95 & 107 & 104 & 105 \\
\hline Ohio State & 64 & 70 & 85 & 98 & 68 & 68 & 79 & 95 & 28 & 28 & 41 & 54 \\
\hline Oklahoma & 109 & 106 & 110 & 107 & 110 & 104 & 110 & 103 & 29 & 28 & 45 & 74 \\
\hline Oklahoma State & 113 & 100 & 101 & 104 & 113 & 103 & 100 & 105 & 85 & 99 & 99 & 105 \\
\hline Oregon & 87 & 90 & 107 & 103 & 87 & 88 & 103 & 102 & 85 & 92 & 104 & 108 \\
\hline Pennsylvania & 42 & 47 & 52 & 66 & 41 & 49 & 48 & 64 & 17 & 28 & 27 & 39 \\
\hline Pennsylvania State & 27 & 30 & 34 & 39 & 30 & 32 & 37 & 38 & 34 & 44 & 45 & 56 \\
\hline Pittsburgh & 66 & 57 & 62 & 61 & 85 & 61 & 69 & 65 & 110 & 113 & 108 & 111 \\
\hline Princeton & 5 & 4 & 16 & 13 & 15 & 7 & 23 & 18 & 17 & 28 & 45 & 16 \\
\hline Purdue & 60 & 44 & 63 & 48 & 82 & 59 & 82 & 72 & 60 & 16 & 23 & 29 \\
\hline Queen's & 74 & 38 & 23 & 17 & 51 & 25 & 14 & 11 & 105 & 96 & 73 & 64 \\
\hline Rice & 71 & 72 & 82 & 72 & 89 & 87 & 92 & 83 & 84 & 97 & 98 & 85 \\
\hline Rochester & 106 & 101 & 102 & 102 & 105 & 99 & 99 & 101 & 63 & 79 & 95 & 103 \\
\hline
\end{tabular}




\section{TABLE 14: AVERAGE, MEDIAN, AND BEGINNING PROFESSIONAL SALARIES IN ARL UNIVERSITY LIBRARIES \\ SUMMARY OF RANKINGS, FYs 2004-2005 TO 2007-2008*}

\begin{tabular}{|c|c|c|c|c|c|c|c|c|c|c|c|c|}
\hline \multirow{2}{*}{$\begin{array}{l}\text { Institution } \\
\text { FY }\end{array}$} & \multicolumn{4}{|c|}{ Average Salaries } & \multicolumn{4}{|c|}{ Median Salaries } & \multicolumn{4}{|c|}{ Beginning Salaries } \\
\hline & 2005 & 2006 & 2007 & 2008 & 2005 & 2006 & 2007 & 2008 & 2005 & 2006 & 2007 & 2008 \\
\hline Rutgers & 4 & 5 & 1 & 1 & 4 & 2 & 1 & 1 & 62 & 7 & 16 & 13 \\
\hline Saskatchewan & 68 & 65 & 31 & 12 & 58 & 52 & 25 & 10 & 106 & 105 & 102 & 53 \\
\hline South Carolina & 98 & 104 & 108 & 110 & 102 & 108 & 106 & 109 & 85 & 99 & 108 & 111 \\
\hline Southern California & 17 & 2 & 21 & 22 & 24 & 19 & 33 & 33 & 3 & 5 & 11 & 16 \\
\hline Southern Illinois & 76 & 92 & 100 & 105 & 94 & 106 & 107 & 107 & 35 & 44 & 61 & 58 \\
\hline SUNY Albany & 72 & 81 & 80 & 77 & 54 & 64 & 68 & 77 & 52 & 64 & 72 & 94 \\
\hline SUNY Buffalo & 81 & 80 & 81 & 58 & 84 & 82 & 84 & 52 & 36 & 49 & 11 & 16 \\
\hline SUNY Stony Brook & 11 & 22 & 22 & 24 & 13 & 20 & 19 & 25 & 14 & 24 & 27 & 34 \\
\hline Syracuse & 50 & 61 & 58 & 53 & 72 & 78 & 74 & 67 & 73 & 79 & 113 & N/A \\
\hline Temple & 86 & 67 & 46 & 45 & 92 & 84 & 57 & 78 & 85 & 76 & 75 & 86 \\
\hline Tennessee & 36 & 43 & 45 & 32 & 42 & 51 & 34 & 30 & 60 & 28 & 45 & 44 \\
\hline Texas & 41 & 51 & 57 & 64 & 55 & 72 & 67 & 73 & 36 & 28 & 17 & 16 \\
\hline Texas A\&M & 79 & 88 & 92 & 79 & 91 & 93 & 96 & 85 & 17 & 28 & 27 & 27 \\
\hline Texas Tech & 94 & 99 & 106 & 81 & 101 & 105 & 105 & 94 & 36 & 51 & 75 & 16 \\
\hline Toronto & 49 & 27 & 4 & 8 & 17 & 12 & 2 & 6 & 93 & 75 & 34 & 33 \\
\hline Tulane & 83 & 89 & 73 & 87 & 77 & 80 & 60 & 80 & 85 & 99 & 84 & 100 \\
\hline Utah & 93 & 94 & 90 & 80 & 93 & 96 & 91 & 88 & 55 & 51 & 45 & 44 \\
\hline Vanderbilt & 100 & 91 & 89 & 94 & 99 & 92 & 95 & 97 & 85 & 79 & 75 & 86 \\
\hline Virginia & 47 & 33 & 33 & 36 & 40 & 28 & 35 & 58 & 29 & 28 & 35 & 34 \\
\hline Virginia Tech & 70 & 75 & 74 & 67 & 65 & 55 & 70 & 61 & 73 & 51 & 84 & 91 \\
\hline Washington & 48 & 59 & 70 & 63 & 56 & 70 & 75 & 70 & 36 & 48 & 45 & 58 \\
\hline Washington State & 88 & 62 & 79 & 91 & 79 & 44 & 77 & 89 & 95 & 68 & 81 & 94 \\
\hline Washington U.-St. Louis & 59 & 64 & 84 & 93 & 88 & 89 & 90 & 99 & 55 & 68 & 84 & 100 \\
\hline Waterloo & 89 & 69 & 30 & 31 & 63 & 29 & 16 & 17 & 109 & 104 & 63 & 52 \\
\hline Wayne State & 32 & 107 & 103 & 109 & 67 & 110 & 109 & 110 & 29 & 79 & 45 & 67 \\
\hline Western Ontario & 112 & 111 & 95 & 88 & 108 & 107 & 98 & 91 & 92 & 67 & 40 & 65 \\
\hline Wisconsin & 61 & 71 & 66 & 83 & 52 & 62 & 58 & 74 & 57 & 63 & 62 & 84 \\
\hline Yale & 3 & 10 & 3 & 6 & 11 & 27 & 13 & 15 & 6 & 27 & 4 & 3 \\
\hline York & 23 & 9 & 6 & 2 & 16 & 14 & 12 & 7 & 104 & 102 & 97 & 32 \\
\hline
\end{tabular}

* Excludes medical and law libraries.

$\uparrow$ Not a member during this year. 
Page intentionally left blank. 
TABLE 15: DISTRIBUTION OF PROFESSIONAL STAFF IN ARL UNIVERSITY LIBRARIES BY SALARY AND POSITION, FY 2007-2008*

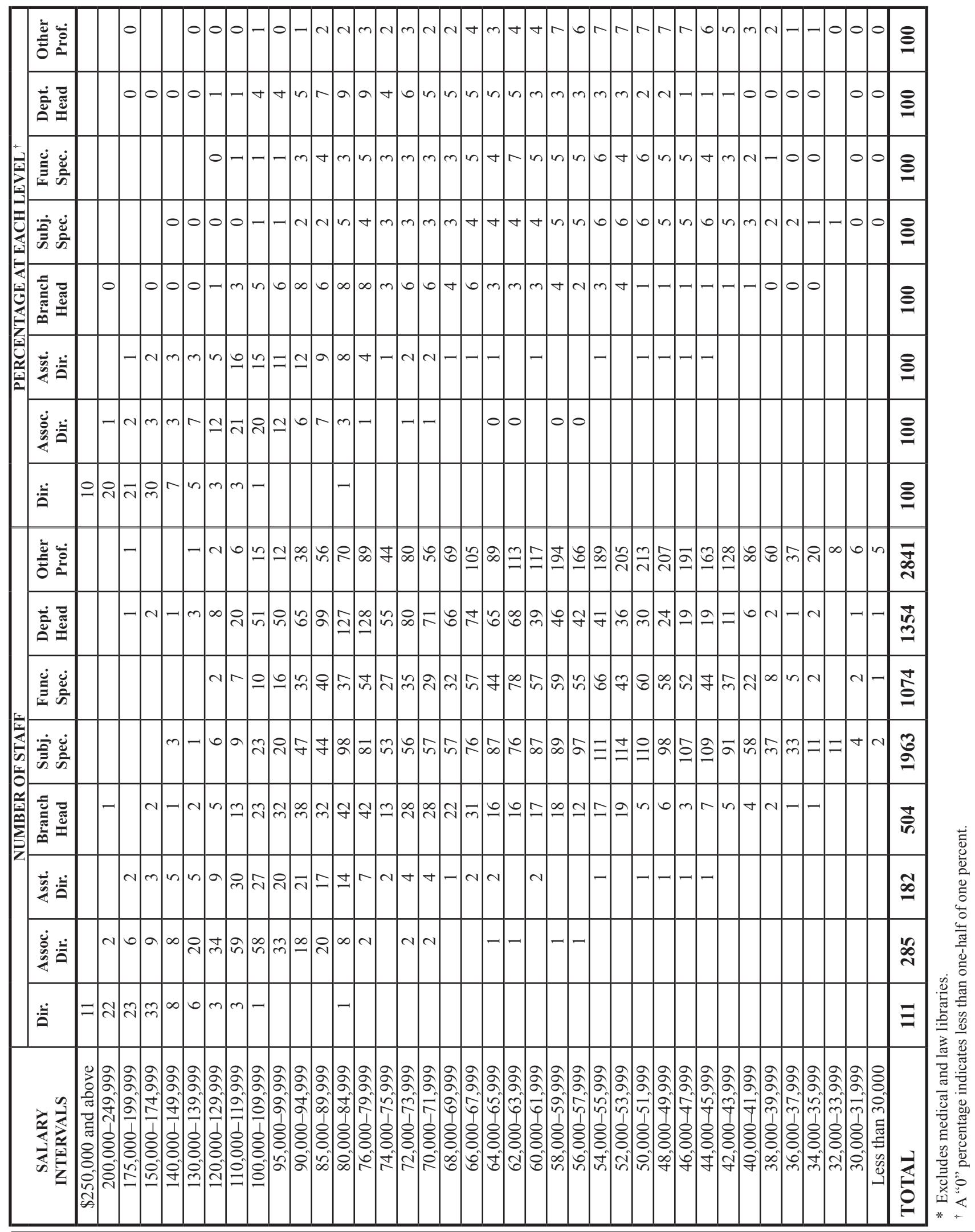

40 · ARL Annual Salary Survey 2007-2008 
TABLE 16: DISTRIBUTION OF PROFESSIONAL STAFF IN ARL UNIVERSITY LIBRARIES BY SALARY, SEX, AND POSITION, FY 2007-2008*

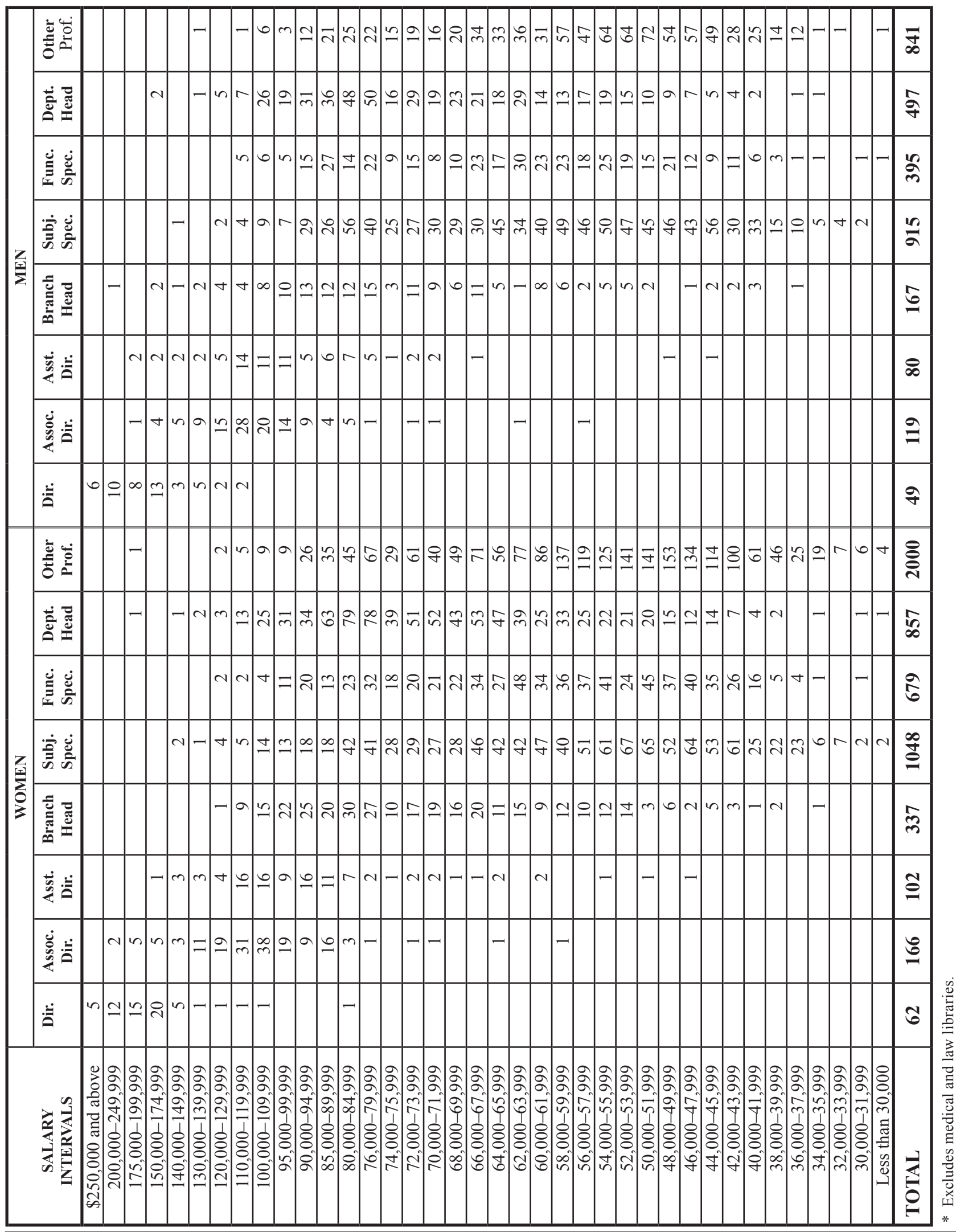


TABLE 17: NUMBER AND AVERAGE SALARIES OF ARL UNIVERSITY LIBRARIANS BY POSITION AND SEX, FY 2007-2008*

\begin{tabular}{|c|c|c|c|c|c|c|c|}
\hline \multirow{2}{*}{ Position } & & \multicolumn{2}{|c|}{ Women } & \multicolumn{2}{|c|}{ Men } & \multicolumn{2}{|c|}{ Total } \\
\hline & & Salary & No. & Salary & No. & Salary & No. \\
\hline \multicolumn{2}{|l|}{ Director } & $\$ 183,287$ & 62 & $\$ 186,383$ & 49 & $\$ 184,654$ & 111 \\
\hline \multicolumn{2}{|c|}{ Associate Director } & 112,045 & 166 & 110,927 & 119 & 111,578 & 285 \\
\hline \multicolumn{2}{|c|}{ Assistant Director } & 97,431 & 102 & 102,028 & 80 & 99,452 & 182 \\
\hline \multicolumn{2}{|c|}{ Head, Branch } & 74,830 & 337 & 79,555 & 167 & 76,396 & 504 \\
\hline \multicolumn{2}{|c|}{ Functional Specialist } & 59,975 & 1048 & 61,634 & 915 & 60,748 & 1963 \\
\hline \multicolumn{2}{|c|}{ Subject Specialist } & 61,568 & 679 & 65,141 & 395 & 62,882 & 1074 \\
\hline \multirow[t]{9}{*}{ Dept. Head: } & Acquisitions & 70,693 & 74 & 69,984 & 31 & 70,484 & 105 \\
\hline & Reference & 75,912 & 82 & 78,594 & 31 & 76,648 & 113 \\
\hline & Cataloging & 72,656 & 119 & 70,881 & 44 & 72,177 & 163 \\
\hline & Serials & 67,900 & 24 & 69,392 & 10 & 68,339 & 34 \\
\hline & Documents/Maps & 67,544 & 44 & 65,918 & 29 & 66,898 & 73 \\
\hline & Circulation & 69,611 & 66 & 63,664 & 28 & 67,839 & 94 \\
\hline & Rare Books/Manuscripts & 79,069 & 44 & 82,152 & 45 & 80,628 & 89 \\
\hline & Computer Systems & 87,107 & 27 & 87,136 & 51 & 87,126 & 78 \\
\hline & Other & 72,447 & 377 & 74,408 & 228 & 73,186 & 605 \\
\hline \multirow[t]{4}{*}{ Reference: } & Over 14 years experience & 64,903 & 441 & 64,626 & 194 & 64,818 & 635 \\
\hline & 10 to 14 years experience & 56,773 & 142 & 55,896 & 60 & 56,513 & 202 \\
\hline & 5 to 9 years experience & 53,088 & 227 & 53,597 & 91 & 53,234 & 318 \\
\hline & Under 5 years experience & 46,039 & 214 & 46,372 & 76 & 46,126 & 290 \\
\hline \multirow[t]{4}{*}{ Cataloging: } & Over 14 years experience & 62,134 & 286 & 62,989 & 137 & 62,411 & 423 \\
\hline & 10 to 14 years experience & 54,679 & 60 & 59,432 & 25 & 56,077 & 85 \\
\hline & 5 to 9 years experience & 52,192 & 91 & 51,846 & 43 & 52,081 & 134 \\
\hline & Under 5 years experience & 46,671 & 93 & 48,456 & 38 & 47,189 & 131 \\
\hline \multirow[t]{4}{*}{ Other: } & Over 14 years experience & 66,282 & 184 & 68,547 & 65 & 66,873 & 249 \\
\hline & 10 to 14 years experience & 58,802 & 54 & 60,269 & 29 & 59,314 & 83 \\
\hline & 5 to 9 years experience & 53,767 & 102 & 54,833 & 48 & 54,108 & 150 \\
\hline & Under 5 years experience & 46,768 & 105 & 45,937 & 35 & 46,560 & 140 \\
\hline \multicolumn{2}{|c|}{ All Positions } & $\$ 66,040$ & 5,250 & $\$ 69,229$ & 3,063 & $\$ 67,215$ & 8,313 \\
\hline
\end{tabular}

* Canadian salaries expressed in U.S. dollars. See Table 32 for salaries of Canadian librarians expressed in Canadian dollars.

Excludes medical and law libraries. See Tables 39 and 46 for salaries in medical and law libraries. 


\section{TABLE 18: NUMBER AND AVERAGE YEARS OF EXPERIENCE OF ARL UNIVERSITY LIBRARIANS \\ BY POSITION AND SEX, FY 2007-2008*}

\begin{tabular}{|c|c|c|c|c|c|c|c|}
\hline \multirow{2}{*}{\multicolumn{2}{|c|}{ Position }} & \multicolumn{2}{|c|}{ Women } & \multicolumn{2}{|c|}{ Men } & \multicolumn{2}{|c|}{ Total } \\
\hline & & Years & No. & Years & No. & Years & No. \\
\hline \multicolumn{2}{|c|}{ Director } & 32.1 & 62 & 31.9 & 49 & 32.0 & 111 \\
\hline \multicolumn{2}{|c|}{ Associate Director } & 25.9 & 166 & 23.3 & 119 & 24.8 & 285 \\
\hline \multicolumn{2}{|c|}{ Assistant Director } & 24.4 & 102 & 23.6 & 80 & 24.0 & 182 \\
\hline \multicolumn{2}{|c|}{ Head, Branch } & 21.8 & 337 & 22.4 & 167 & 22.0 & 504 \\
\hline \multicolumn{2}{|c|}{ Functional Specialist } & 13.8 & 1,048 & 12.7 & 915 & 13.2 & 1,963 \\
\hline \multicolumn{2}{|c|}{ Subject Specialist } & 16.1 & 679 & 17.3 & 395 & 16.6 & 1,074 \\
\hline \multirow[t]{9}{*}{ Dept. Head: } & Acquisitions & 21.0 & 74 & 19.2 & 31 & 20.5 & 105 \\
\hline & Reference & 20.3 & 81 & 21.6 & 31 & 20.7 & 112 \\
\hline & Cataloging & 23.2 & 119 & 23.5 & 44 & 23.2 & 163 \\
\hline & Serials & 20.1 & 24 & 22.5 & 10 & 20.8 & 34 \\
\hline & Documents/Maps & 20.0 & 44 & 20.0 & 29 & 20.0 & 73 \\
\hline & Circulation & 21.0 & 66 & 16.6 & 28 & 19.7 & 94 \\
\hline & Rare Books/Manuscripts & 22.4 & 44 & 24.3 & 44 & 23.3 & 88 \\
\hline & Computer Systems & 18.8 & 27 & 18.8 & 51 & 18.8 & 78 \\
\hline & Other & 20.1 & 376 & 19.6 & 228 & 19.9 & 604 \\
\hline \multicolumn{2}{|c|}{ Public Services } & 12.3 & 184 & 13.1 & 74 & 12.5 & 258 \\
\hline \multicolumn{2}{|c|}{ Technical Services } & 14.9 & 123 & 14.1 & 52 & 14.7 & 175 \\
\hline \multicolumn{2}{|c|}{ Administrative Services } & 15.4 & 138 & 14.0 & 51 & 15.0 & 189 \\
\hline \multicolumn{2}{|c|}{ Reference } & 14.4 & 1,024 & 14.9 & 421 & 14.6 & 1,445 \\
\hline \multicolumn{2}{|l|}{ Cataloger } & 17.2 & 530 & 17.6 & 243 & 17.3 & 773 \\
\hline \multicolumn{2}{|c|}{ All Positions } & 17.0 & 5,248 & 16.8 & 3,062 & 16.9 & 8,310 \\
\hline
\end{tabular}

* Includes Canadian libraries. See Table 33 for comparable figures in Canadian libraries only.

Excludes medical and law libraries. See Tables 40 and 47 for comparable figures in medical and law libraries. 
TABLE 19: NUMBER AND AVERAGE SALARIES OF ARL UNIVERSITY LIBRARIANS BY YEARS OF EXPERIENCE AND SEX, FY 2007-2008*

\begin{tabular}{lccccccc}
\hline & \multicolumn{2}{c}{ Women } & \multicolumn{2}{c}{ Men } & \multicolumn{2}{c}{ Total } & \multicolumn{2}{c}{ of } \\
Experience & Salary & No. & Salary & No. & Salary & No. & Total \\
\hline $0-3$ years & $\$ 47,152$ & 644 & $\$ 49,753$ & 325 & $\$ 48,024$ & 969 & $12 \%$ \\
$4-7$ years & 52,607 & 702 & 55,089 & 467 & 53,599 & 1,169 & $14 \%$ \\
$8-11$ years & 58,493 & 683 & 61,504 & 426 & 59,650 & 1,109 & $13 \%$ \\
$12-15$ years & 62,945 & 546 & 66,363 & 335 & 64,245 & 881 & $11 \%$ \\
$16-19$ years & 67,358 & 552 & 69,572 & 326 & 68,180 & 878 & $11 \%$ \\
$20-23$ years & 72,339 & 526 & 75,884 & 281 & 73,573 & 807 & $10 \%$ \\
$24-27$ years & 75,916 & 470 & 80,071 & 257 & 77,385 & 727 & $9 \%$ \\
$28-31$ years & 79,225 & 439 & 85,981 & 257 & 81,719 & 696 & $8 \%$ \\
$32-35$ years & 86,463 & 378 & 88,588 & 243 & 87,294 & 621 & $7 \%$ \\
over 35 years & 86,339 & 308 & 92,667 & 145 & 88,364 & 453 & $5 \%$ \\
\hline All Positions & $\mathbf{\$ 6 6 , 0 4 1}$ & $\mathbf{5 , 2 4 8}$ & $\mathbf{\$ 6 9 , 2 2 6}$ & $\mathbf{3 , 0 6 2}$ & $\mathbf{\$ 6 7 , 2 1 5}$ & $\mathbf{8 , 3 1 0}$ & $\mathbf{1 0 0 \%}$ \\
\hline
\end{tabular}

* Canadian salaries expressed in U.S. dollars. See Table 34 for salaries in Canadian dollars.

Excludes medical and law libraries. See Tables 41 and 48 for salaries in medical and law libraries. 


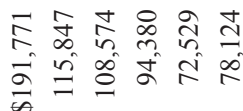

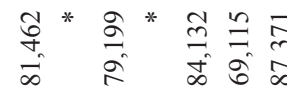

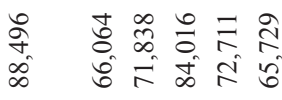

나요

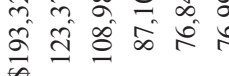

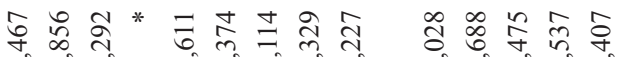

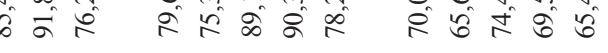

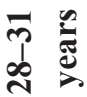

:

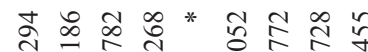

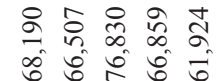

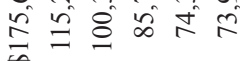

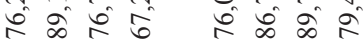

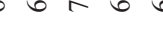

సิ

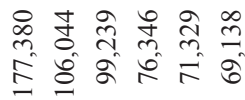

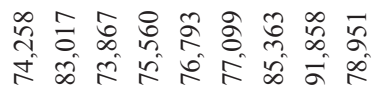

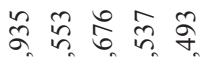

औी

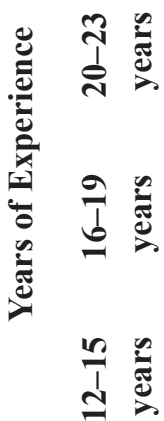

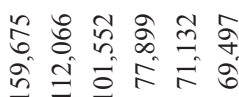

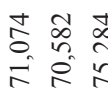

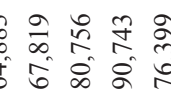

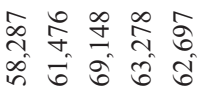

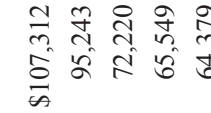

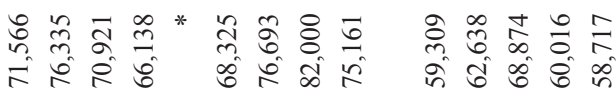

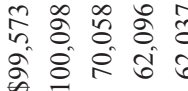

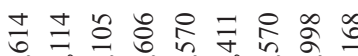

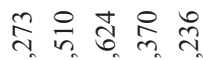
के

$=$

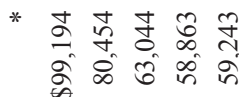

क कि

$\frac{\infty}{\infty}$

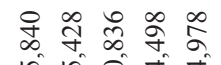

in in 8 in in

F

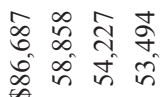

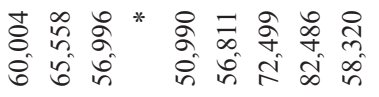

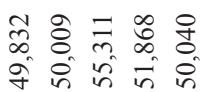

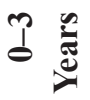

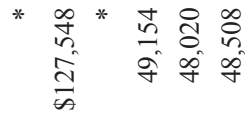

点*

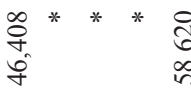

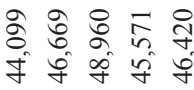

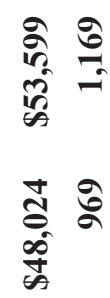

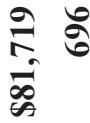

点

$\overbrace{\substack{n \\ n}}^{\infty} \hat{a}$

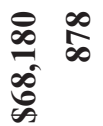

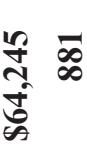

(8)

:

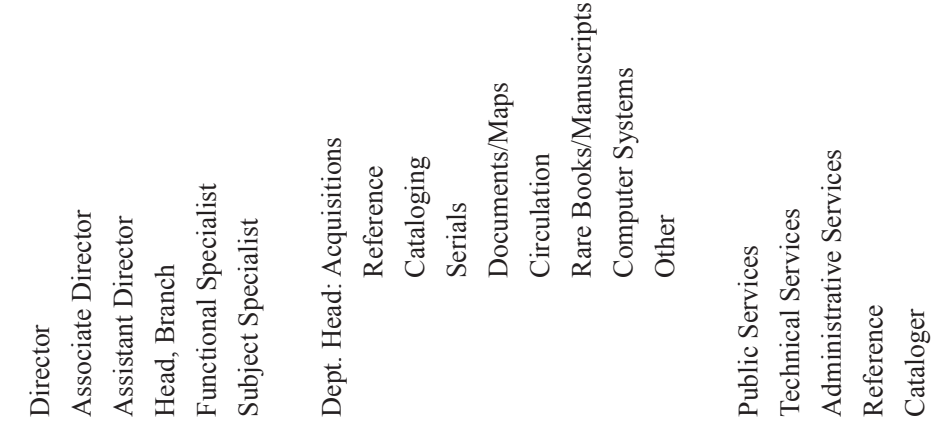

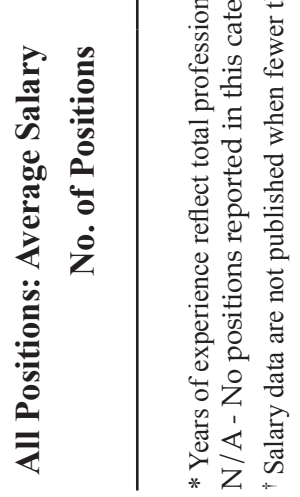


TABLE 21: NUMBER AND AVERAGE SALARIES OF ARL UNIVERSITY LIBRARIANS BY POSITION AND TYPE OF INSTITUTION, FY 2007-2008*

\begin{tabular}{|c|c|c|c|c|c|c|c|c|}
\hline \multirow[b]{2}{*}{ Position } & \multicolumn{2}{|c|}{ Canadian (14) } & \multicolumn{2}{|c|}{ Private (31) } & \multicolumn{2}{|c|}{ Public (68) } & \multicolumn{2}{|c|}{ Total (113) } \\
\hline & Salary & No. & Salary & No. & Salary & No. & Salary & No. \\
\hline Director & $\$ 139,594$ & 14 & $\$ 215,741$ & 31 & $\$ 179,611$ & 66 & $\$ 184,654$ & 111 \\
\hline Associate Director & 102,608 & 34 & 121,525 & 87 & 108,161 & 164 & 111,578 & 285 \\
\hline Assistant Director & 83,991 & 22 & 104,013 & 67 & 99,823 & 93 & 99,452 & 182 \\
\hline Head, Branch & 82,937 & 59 & 81,593 & 135 & 72,888 & 310 & 76,396 & 504 \\
\hline Functional Specialist & 63,357 & 133 & 63,699 & 739 & 58,431 & 1091 & 60,748 & 1963 \\
\hline Subject Specialist & 65,868 & 81 & 64,464 & 381 & 61,502 & 612 & 62,882 & 1074 \\
\hline Dept. Head: Acquisitions & 74,342 & 12 & 71,993 & 30 & 69,030 & 63 & 70,484 & 105 \\
\hline Reference & 73,102 & 12 & 78,099 & 46 & 76,207 & 55 & 76,648 & 113 \\
\hline Cataloging & 80,201 & 10 & 73,583 & 69 & 70,067 & 84 & 72,177 & 163 \\
\hline Serials & $*$ & 3 & 68,218 & 11 & 66,851 & 20 & 68,339 & 34 \\
\hline Documents/Maps & 84,306 & 8 & 64,417 & 14 & 64,848 & 51 & 66,898 & 73 \\
\hline Circulation & 69,737 & 14 & 64,553 & 30 & 69,280 & 50 & 67,839 & 94 \\
\hline Rare Books/Manuscripts & 83,543 & 7 & 78,556 & 25 & 81,178 & 57 & 80,628 & 89 \\
\hline Computer Systems & 78,770 & 7 & 91,298 & 27 & 85,896 & 44 & 87,126 & 78 \\
\hline Other & 77,266 & 47 & 75,676 & 196 & 71,308 & 362 & 73,186 & 605 \\
\hline Reference: Over 14 years experience & 75,356 & 112 & 62,559 & 165 & 62,562 & 358 & 64,818 & 635 \\
\hline 10 to 14 years experience & 65,868 & 23 & 56,740 & 52 & 54,725 & 127 & 56,513 & 202 \\
\hline 5 to 9 years experience & 58,128 & 66 & 54,995 & 75 & 50,662 & 177 & 53,234 & 318 \\
\hline Under 5 years experience & 50,207 & 71 & 46,261 & 55 & 44,313 & 164 & 46,126 & 290 \\
\hline Cataloging: Over 14 years experience & 72,146 & 36 & 62,769 & 168 & 60,536 & 219 & 62,411 & 423 \\
\hline 10 to 14 years experience & 52,677 & 5 & 57,981 & 31 & 55,219 & 49 & 56,077 & 85 \\
\hline 5 to 9 years experience & 56,059 & 12 & 54,085 & 71 & 48,356 & 51 & 52,081 & 134 \\
\hline Under 5 years experience & 60,014 & 5 & 49,275 & 50 & 44,973 & 76 & 47,189 & 131 \\
\hline Other: Over 14 years experience & 69,943 & 12 & 68,492 & 92 & 65,592 & 145 & 66,873 & 249 \\
\hline 10 to 14 years experience & 62,719 & 6 & 62,742 & 33 & 56,279 & 44 & 59,314 & 83 \\
\hline 5 to 9 years experience & 54,454 & 11 & 59,753 & 51 & 50,794 & 88 & 54,108 & 150 \\
\hline Under 5 years experience & 56,080 & 6 & 49,019 & 53 & 44,246 & 81 & 46,560 & 140 \\
\hline All Positions & $\$ 70,667$ & 828 & $\$ 69,384$ & 2,784 & $\$ 65,323$ & 4,701 & $\$ 67,215$ & 8,313 \\
\hline
\end{tabular}

* Canadian salaries expressed in U.S. dollars. Tables 31-34 show Canadian salaries in Canadian dollars. Excludes medical and law libraries.

( ) Indicates the number of ARL libraries in each category.

¥ Salary data are not published when fewer than four individuals are involved. 


\section{TABLE 22: YEARS OF EXPERIENCE OF ARL UNIVERSITY LIBRARIANS BY POSITION AND TYPE OF INSTITUTION, FY 2007-2008*}

\begin{tabular}{|c|c|c|c|c|c|c|c|c|}
\hline \multirow[b]{2}{*}{ Position } & \multicolumn{2}{|c|}{ Canadian (14) } & \multicolumn{2}{|c|}{ Private (31) } & \multicolumn{2}{|c|}{ Public (68) } & \multicolumn{2}{|c|}{ Total (113) } \\
\hline & Years & No. & Years & No. & Years & No. & Years & No. \\
\hline Director & 28.9 & 14 & 32.7 & 31 & 32.3 & 66 & 32.0 & 111 \\
\hline Associate Director & 23.8 & 34 & 25.4 & 87 & 24.7 & 164 & 24.8 & 285 \\
\hline Assistant Director & 20.3 & 22 & 24.4 & 67 & 24.6 & 93 & 24.0 & 182 \\
\hline Head, Branch & 22.6 & 59 & 23.3 & 135 & 21.3 & 310 & 22.0 & 504 \\
\hline Functional Specialist & 14.6 & 133 & 13.2 & 739 & 13.1 & 1091 & 13.2 & 1963 \\
\hline Subject Specialist & 17.0 & 81 & 17.1 & 381 & 16.2 & 612 & 16.6 & 1074 \\
\hline Dept. Head: Acquisitions & 19.9 & 12 & 22.1 & 30 & 19.8 & 63 & 20.5 & 105 \\
\hline Reference & 18.1 & 12 & 21.5 & 46 & 20.5 & 54 & 20.7 & 112 \\
\hline Cataloging & 26.0 & 10 & 23.3 & 69 & 22.9 & 84 & 23.2 & 163 \\
\hline Serials & 23.7 & 3 & 22.5 & 11 & 19.5 & 20 & 20.8 & 34 \\
\hline Documents/Maps & 24.9 & 8 & 18.0 & 14 & 19.8 & 51 & 20.0 & 73 \\
\hline Circulation & 19.4 & 14 & 19.6 & 30 & 19.9 & 50 & 19.7 & 94 \\
\hline Rare Books/Manuscripts & 27.1 & 7 & 19.8 & 25 & 24.4 & 56 & 23.3 & 88 \\
\hline Computer Systems & 21.9 & 7 & 18.8 & 27 & 18.3 & 44 & 18.8 & 78 \\
\hline Other & 18.7 & 47 & 20.6 & 196 & 19.7 & 361 & 19.9 & 604 \\
\hline Reference: Over 14 years experience & 25.3 & 112 & 25.1 & 165 & 24.6 & 358 & 24.8 & 635 \\
\hline 10 to 14 years experience & 11.9 & 23 & 11.8 & 52 & 11.9 & 127 & 11.9 & 202 \\
\hline 5 to 9 years experience & 6.8 & 66 & 7.0 & 75 & 6.9 & 177 & 6.9 & 318 \\
\hline Under 5 years experience & 2.5 & 71 & 2.1 & 55 & 2.4 & 164 & 2.4 & 290 \\
\hline Cataloging: Over 14 years experience & 28.2 & 36 & 25.7 & 168 & 26.4 & 219 & 26.3 & 423 \\
\hline 10 to 14 years experience & 11.4 & 5 & 12.1 & 31 & 12.1 & 49 & 12.0 & 85 \\
\hline 5 to 9 years experience & 7.3 & 12 & 7.0 & 71 & 6.8 & 51 & 6.9 & 134 \\
\hline Under 5 years experience & 3.0 & 5 & 2.5 & 50 & 2.3 & 76 & 2.4 & 131 \\
\hline Other: Over 14 years experience & 24.8 & 12 & 24.4 & 92 & 25.9 & 145 & 25.3 & 249 \\
\hline 10 to 14 years experience & 12.3 & 6 & 11.8 & 33 & 12.0 & 44 & 11.9 & 83 \\
\hline 5 to 9 years experience & 6.8 & 11 & 6.7 & 51 & 7.1 & 88 & 7.0 & 150 \\
\hline Under 5 years experience & 3.3 & 6 & 2.2 & 53 & 2.1 & 81 & 2.2 & 140 \\
\hline All Positions & 17.1 & 828 & 17.0 & 2,784 & 16.8 & 4,698 & 16.9 & 8,310 \\
\hline
\end{tabular}

* Excludes medical and law libraries.

( ) Indicates the number of ARL libraries in each category. 
TABLE 23: NUMBER AND AVERAGE SALARIES OF ARL UNIVERSITY LIBRARIANS BY POSITION AND SIZE OF PROFESSIONAL STAFF, FY 2007-2008*

\begin{tabular}{|c|c|c|c|c|c|c|c|c|}
\hline \multirow[b]{2}{*}{ Position } & \multicolumn{2}{|c|}{ Staff Over $110(16)$} & \multicolumn{2}{|c|}{ Staff $75-110(21)$} & \multicolumn{2}{|c|}{ Staff 50-74 (46) } & \multicolumn{2}{|c|}{ Staff $22-49(30) \S$} \\
\hline & Salary & No. & Salary & No. & Salary & No. & Salary & No. \\
\hline Director & $\$ 224,990$ & 16 & $\$ 191,500$ & 21 & $\$ 179,011$ & 44 & $\$ 166,625$ & 30 \\
\hline Associate Director & 132,578 & 57 & 113,727 & 68 & 104,382 & 101 & 101,130 & 59 \\
\hline Assistant Director & 107,503 & 45 & 96,730 & 47 & 97,671 & 69 & 94,139 & 21 \\
\hline Head, Branch & 81,686 & 161 & 79,364 & 90 & 71,696 & 171 & 72,551 & 82 \\
\hline Functional Specialist & 63,605 & 721 & 61,144 & 464 & 58,344 & 557 & 56,657 & 221 \\
\hline Subject Specialist & 67,303 & 347 & 62,729 & 295 & 60,105 & 335 & 57,127 & 97 \\
\hline Dept. Head: Acquisitions & 73,850 & 18 & 81,425 & 20 & 64,424 & 37 & 68,643 & 30 \\
\hline Reference & 82,725 & 32 & 77,620 & 15 & 70,991 & 40 & 77,310 & 26 \\
\hline Cataloging & 75,770 & 60 & 74,239 & 29 & 66,988 & 53 & 72,156 & 21 \\
\hline Serials & 74,978 & 11 & 69,938 & 4 & 63,630 & 12 & 65,064 & 7 \\
\hline Documents/Maps & 69,123 & 22 & 70,233 & 12 & 66,494 & 24 & 61,612 & 15 \\
\hline Circulation & 74,447 & 24 & 66,631 & 21 & 65,473 & 35 & 64,239 & 14 \\
\hline Rare Books/Manuscripts & 92,976 & 18 & 82,142 & 13 & 75,925 & 33 & 77,157 & 25 \\
\hline Computer Systems & 102,651 & 8 & 92,719 & 18 & 83,800 & 36 & 80,557 & 16 \\
\hline Other & 77,602 & 160 & 76,577 & 134 & 69,322 & 233 & 69,846 & 78 \\
\hline Reference: Over 14years experience & 66,643 & 145 & 66,284 & 120 & 63,595 & 230 & 63,680 & 140 \\
\hline 10 to 14 years experience & 57,139 & 45 & 57,432 & 37 & 55,732 & 84 & 56,606 & 36 \\
\hline 5 to 9 years experience & 57,036 & 82 & 53,596 & 63 & 51,396 & 114 & 51,114 & 59 \\
\hline Under 5 years experience & 47,985 & 45 & 47,623 & 62 & 45,297 & 121 & 44,897 & 62 \\
\hline Cataloging: Over 14 years experience & 65,978 & 165 & 60,955 & 87 & 58,610 & 129 & 63,085 & 42 \\
\hline 10 to 14 years experience & 61,087 & 23 & 55,938 & 24 & 54,020 & 27 & 50,951 & 11 \\
\hline 5 to 9 years experience & 55,915 & 56 & 50,626 & 28 & 48,106 & 35 & 49,760 & 15 \\
\hline Under 5 years experience & 49,734 & 60 & 47,183 & 20 & 44,530 & 38 & 43,225 & 13 \\
\hline Other: Over 14 years experience & 68,043 & 92 & 69,143 & 53 & 64,348 & 78 & 65,686 & 26 \\
\hline 10 to 14 years experience & 58,099 & 26 & 61,980 & 29 & 58,574 & 21 & 55,007 & 7 \\
\hline 5 to 9 years experience & 58,098 & 50 & 54,406 & 44 & 51,451 & 41 & 47,200 & 15 \\
\hline Under 5 years experience & 51,050 & 45 & 46,845 & 29 & 43,781 & 57 & 40,797 & 9 \\
\hline All Positions & $\$ 69,603$ & 2,534 & $\$ 67,902$ & 1,847 & $\$ 64,990$ & 2,755 & $\$ 66,204$ & 1,177 \\
\hline
\end{tabular}

*Canadian salaries expressed in U.S. dollars. For average Canadian salaries (expressed in U.S. dollars) refer to Table 21; Tables 31-34 show Canadian salaries in Canadian dollars. Excludes medical and law libraries.

( ) Indicates the number of ARL libraries in each category.

¥In 1995-1996 and earlier, the first column of this table reported staff over 124; in 1996-1998 over 120; in 1998-1999 over 115; and since 1999-2000, over 110.

$\S$ No ARL Library has fewer than 21 professional staff members. 


\section{TABLE 24: YEARS OF EXPERIENCE OF ARL UNIVERSITY LIBRARIANS BY POSITION AND SIZE OF PROFESSIONAL STAFF, FY 2007-2008*}

\begin{tabular}{|c|c|c|c|c|c|c|c|c|}
\hline \multirow[b]{2}{*}{ Position } & \multicolumn{2}{|c|}{ Staff Over $110(16) \dagger$} & \multicolumn{2}{|c|}{ Staff 75-110 (21) } & \multicolumn{2}{|c|}{ Staff 50-74 (46) } & \multicolumn{2}{|c|}{ Staff $24-49(30)$} \\
\hline & Years & No. & Years & No. & Years & No. & Years & No. \\
\hline Director & 32.8 & 16 & 28.5 & 21 & 32.3 & 44 & 33.6 & 30 \\
\hline Associate Director & 26.9 & 57 & 23.7 & 68 & 24.5 & 101 & 24.6 & 59 \\
\hline Assistant Director & 24.0 & 45 & 22.4 & 47 & 24.5 & 69 & 26.2 & 21 \\
\hline Head, Branch & 23.0 & 161 & 21.4 & 90 & 21.5 & 171 & 21.7 & 82 \\
\hline Functional Specialist & 12.6 & 721 & 14.3 & 464 & 13.0 & 557 & 13.8 & 221 \\
\hline Subject Specialist & 16.8 & 347 & 16.0 & 295 & 17.0 & 335 & 15.8 & 97 \\
\hline Dept. Head: Acquisitions & 24.1 & 18 & 24.0 & 20 & 17.4 & 37 & 19.8 & 30 \\
\hline Reference & 23.5 & 31 & 18.8 & 15 & 20.0 & 40 & 19.2 & 26 \\
\hline Cataloging & 23.8 & 60 & 22.9 & 29 & 23.0 & 53 & 22.8 & 21 \\
\hline Serials & 24.4 & 11 & 14.5 & 4 & 17.3 & 12 & 24.9 & 7 \\
\hline Documents/Maps & 22.6 & 22 & 19.9 & 12 & 18.9 & 24 & 18.0 & 15 \\
\hline Circulation & 21.0 & 24 & 17.4 & 21 & 19.4 & 35 & 21.8 & 14 \\
\hline Rare Books/Manuscripts & 25.2 & 17 & 22.8 & 13 & 22.3 & 33 & 23.6 & 25 \\
\hline Computer Systems & 22.1 & 8 & 19.6 & 18 & 17.1 & 36 & 20.3 & 16 \\
\hline Other & 21.1 & 159 & 19.7 & 134 & 18.9 & 233 & 20.6 & 78 \\
\hline Reference: Over 14 years experience & 25.3 & 145 & 24.7 & 120 & 24.7 & 230 & 24.8 & 140 \\
\hline 10 to 14 years experience & 11.8 & 45 & 11.8 & 37 & 11.9 & 84 & 11.9 & 36 \\
\hline 5 to 9 years experience & 6.9 & 82 & 6.6 & 63 & 7.0 & 114 & 7.0 & 59 \\
\hline Under 5 years experience & 2.8 & 45 & 2.4 & 62 & 2.1 & 121 & 2.4 & 62 \\
\hline Cataloging: Over 14 years experience & 26.1 & 165 & 26.6 & 87 & 26.6 & 129 & 25.2 & 42 \\
\hline 10 to 14 years experience & 12.1 & 23 & 12.0 & 24 & 11.9 & 27 & 12.3 & 11 \\
\hline 5 to 9 years experience & 7.0 & 56 & 6.9 & 28 & 6.9 & 35 & 6.9 & 15 \\
\hline Under 5 years experience & 2.4 & 60 & 2.7 & 20 & 2.3 & 38 & 1.8 & 13 \\
\hline Other: Over 14 years experience & 25.8 & 92 & 23.9 & 53 & 25.7 & 78 & 24.8 & 26 \\
\hline 10 to 14 years experience & 11.6 & 26 & 12.2 & 29 & 12.0 & 21 & 12.0 & 7 \\
\hline 5 to 9 years experience & 6.9 & 50 & 7.0 & 44 & 7.1 & 41 & 6.5 & 15 \\
\hline Under 5 years experience & 2.2 & 45 & 2.2 & 29 & 2.0 & 57 & 2.8 & 9 \\
\hline All Positions & 16.9 & 2,531 & 16.6 & 1,847 & 16.9 & 2,755 & 17.7 & 1,177 \\
\hline
\end{tabular}

* Excludes medical and law libraries.

( ) Indicates the number of ARL libraries in each category.

${ }^{\dagger}$ In 1995-1996 and earlier, the first column of this table reported staff over 124; in 1996-1998, over 120; in 1998-1999, over 115; and since 19992000 , over 110.

* No ARL library has fewer than 21 professional staff members. 

AND GEOGRAPHIC REGION, FY 2007-2008*

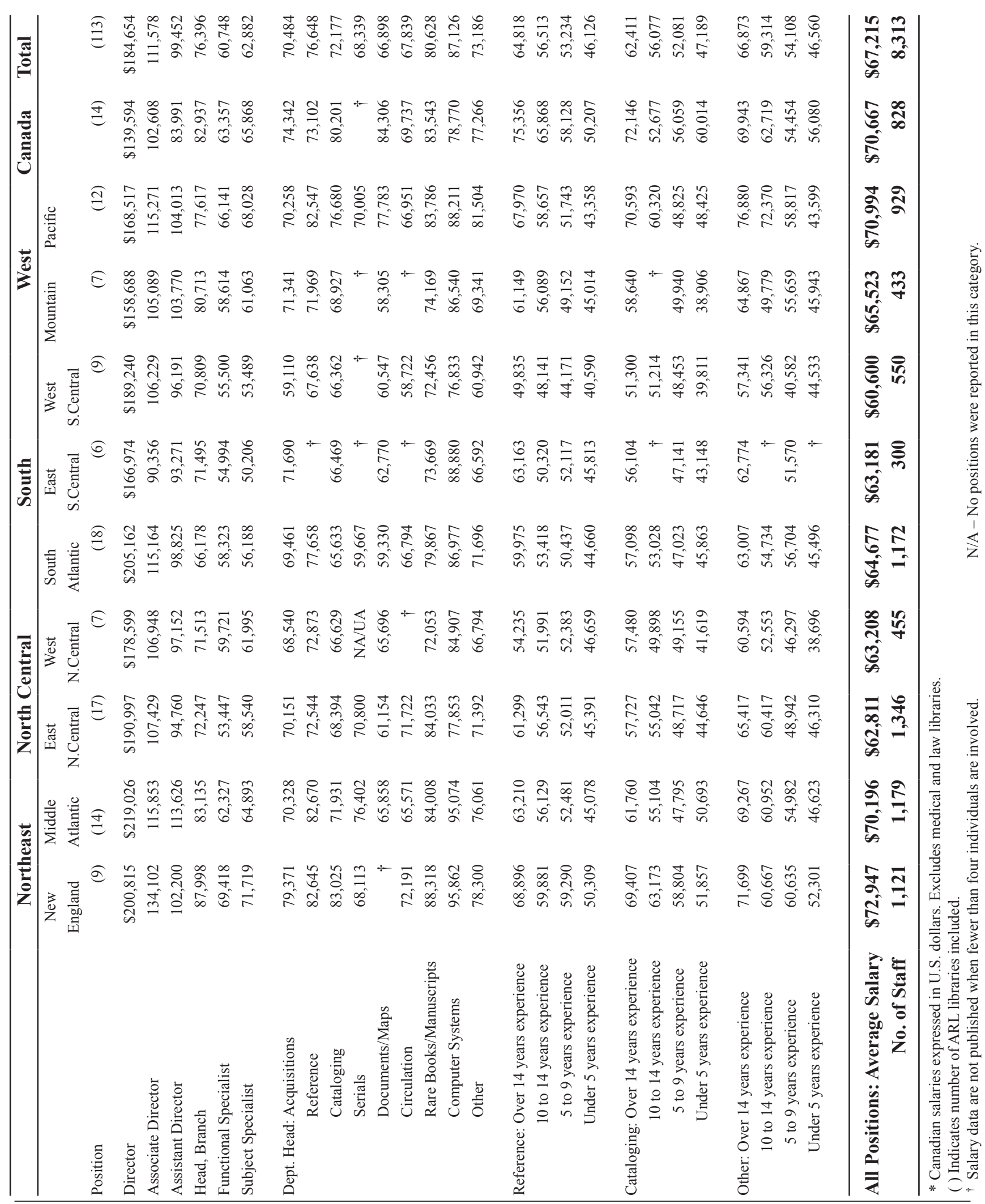


Region

No. of

ARL University Libraries Included

States/Provinces Included

Libraries

\section{Northeast}

1. New England

2. Middle Atlantic

\section{North Central}

3. East North Central

4. West North Central

\section{South}

5. South Atlantic

6. East South Central

7. West South Central

\section{West}

8. Mountain

9. Pacific

Canada
Boston University, Boston College, Brown, Connecticut, Dartmouth, Harvard, Massachusetts Institute of Technology, Massachusetts, Yale

Columbia; Cornell; New York; Pennsylvania; Pennsylvania State; Pittsburgh; Princeton; Rochester; Rutgers; State University of New York: Albany, Buffalo, Stony Brook; Syracuse; Temple

Case Western Reserve, Chicago, Cincinnati, Illinois-Chicago, Illinois-Urbana, Indiana, Kent State, Michigan, Michigan State, Notre Dame, Northwestern, Ohio University, Ohio State, Purdue, Southern Illinois, Wayne State, Wisconsin

Iowa, Iowa State, Kansas, Minnesota, Missouri, Nebraska, Washington U.-St. Louis

Delaware, Duke, Emory, Florida, Florida State, Georgia, Georgia Tech., Georgetown, George Washington, Howard, Johns Hopkins, Maryland, Miami, North Carolina, North Carolina State, South Carolina, Virginia, Virginia Tech

Alabama, Auburn, Kentucky, Louisville, Tennessee, Vanderbilt

Houston, Louisiana State, Oklahoma, Oklahoma State, Rice, Texas, Texas A\&M, Texas Tech, Tulane

Arizona, Arizona State, Brigham Young, Colorado, Colorado State, New Mexico, Utah University of California: Berkeley, Davis, Irvine, Los Angeles, Riverside, San Diego, Santa Barbara; Hawaii; Oregon; Southern California; Washington; Washington State

Alberta, British Columbia, Guelph, Laval, McGill, McMaster, Manitoba, Montreal, Queen's, Saskatchewan, Toronto, Waterloo, Western Ontario, York
Conn., Mass., Me., N.H., R.I., Vt.

N.J., N.Y., Pa.

Ill., Ind., Mich., Ohio, Wis.

Iowa, Kan., Minn., Mo., Neb., N. Dak., S. Dak.

Del., D.C., Fla., Ga., Md., N.C., S.C., Va., W. Va.

Ala., Ky., Miss., Tenn.

Ark., La., Okla., Tex.

Ariz., Colo., Idaho, Mont., Nev., N. Mex., Utah, Wyo.

Alaska, Calif., Hawaii, Ore., Wash.

Alta., B.C., Man., N. Br., Newf., N.S., Ont., P.E.I., Que., Sask.

* Regions are based on the classification used by the U.S. Bureau of the Census in tabulations of the Current Population Survey. 

U.S. ARL UNIVERSITY LIBRARIES

Tables 26-30 

BY POSITION AND YEARS OF EXPERIENCE, FY 2007-2008*

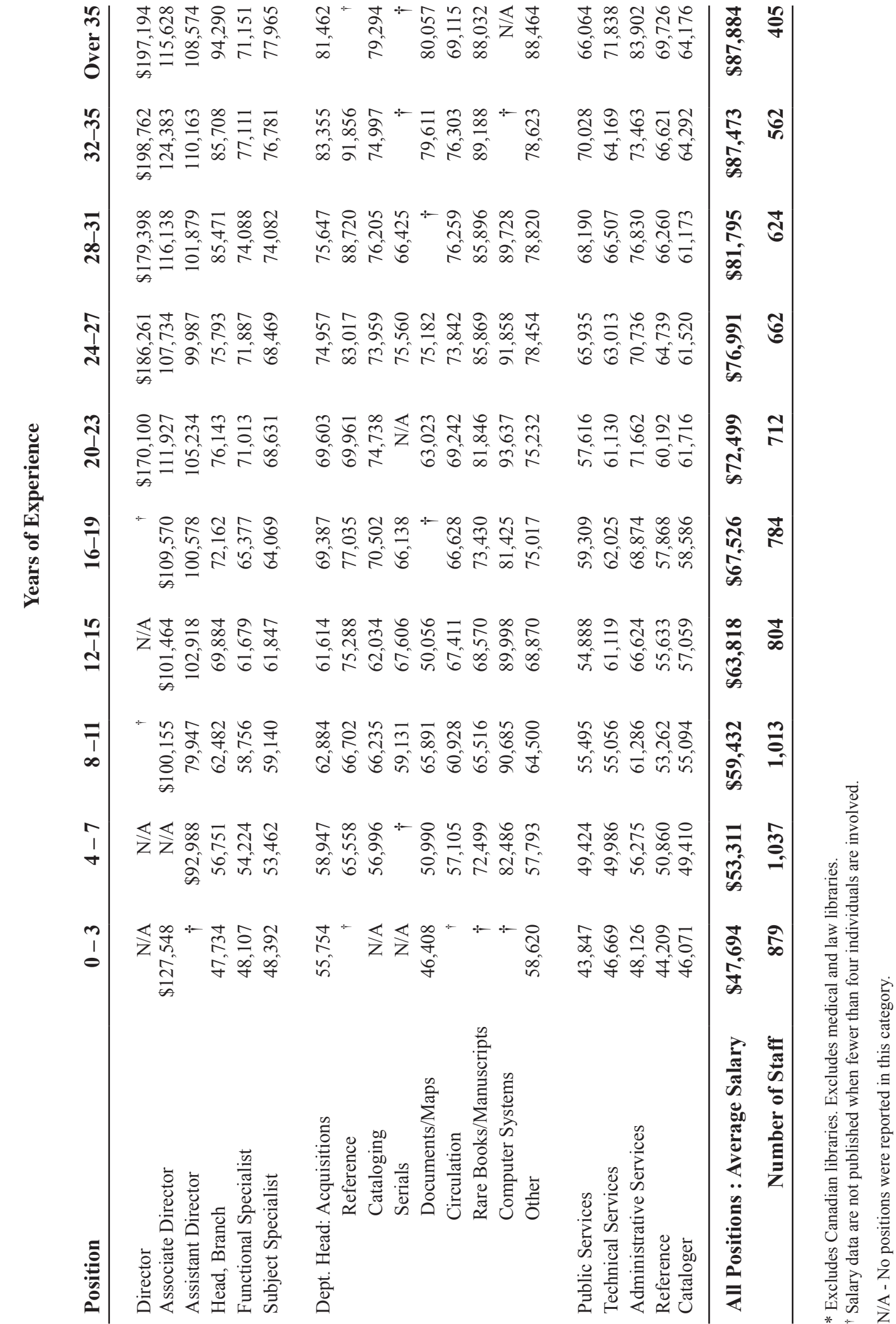




\section{TABLE 27: NUMBER AND AVERAGE SALARIES OF MINORITY U.S. ARL UNIVERSITY LIBRARIANS \\ BY POSITION AND SEX, FY 2007-2008*}

\begin{tabular}{|c|c|c|c|c|c|c|c|}
\hline \multirow{2}{*}{ Position } & & \multicolumn{2}{|c|}{ Women } & \multicolumn{2}{|c|}{ Men } & \multicolumn{2}{|c|}{ Total } \\
\hline & & Salary & No. & Salary & No. & Salary & No. \\
\hline \multicolumn{2}{|l|}{ Director } & $\dagger$ & 3 & $\dagger$ & 2 & $\$ 165,796$ & 5 \\
\hline \multicolumn{2}{|c|}{ Associate Director } & $\$ 115,845$ & 17 & $\$ 95,512$ & 4 & 111,972 & 21 \\
\hline \multicolumn{2}{|c|}{ Assistant Director } & $\dagger$ & 3 & $\dagger$ & 2 & 116,389 & 5 \\
\hline \multicolumn{2}{|c|}{ Head, Branch } & 67,569 & 40 & 77,643 & 12 & 69,894 & 52 \\
\hline \multicolumn{2}{|c|}{ Functional Specialist } & 57,472 & 148 & 59,230 & 120 & 58,259 & 268 \\
\hline \multicolumn{2}{|c|}{ Subject Specialist } & 59,787 & 140 & 60,398 & 53 & 59,955 & 193 \\
\hline \multirow[t]{9}{*}{ Dept. Head: } & Acquisitions & $\dagger$ & 5 & $\dagger$ & 2 & 74,999 & 7 \\
\hline & Reference & $\dagger$ & 5 & $\dagger$ & 1 & 77,879 & 6 \\
\hline & Cataloging & $\dagger$ & 10 & $\dagger$ & 1 & 76,920 & 11 \\
\hline & Serials & $\dagger$ & 3 & N/A & N/A & $\dagger$ & 3 \\
\hline & Documents/Maps & $\dagger$ & 5 & $\dagger$ & 2 & 61,483 & 7 \\
\hline & Circulation & 60,873 & 7 & 67,939 & 6 & 64,134 & 13 \\
\hline & Rare Books/Manuscripts & $\dagger$ & 6 & $\dagger$ & 1 & 73,602 & 7 \\
\hline & Computer Systems & $\dagger$ & 2 & $\dagger$ & 10 & 79,966 & 12 \\
\hline & Other & 76,487 & 32 & 73,309 & 14 & 75,520 & 46 \\
\hline \multirow[t]{4}{*}{ Reference: } & Over 14 years experience & 62,003 & 55 & 63,771 & 18 & 62,439 & 73 \\
\hline & 10 to 14 years experience & 56,487 & 16 & 53,502 & 6 & 55,673 & 22 \\
\hline & 5 to 9 years experience & 49,922 & 18 & 54,805 & 9 & 51,550 & 27 \\
\hline & Under 5 years experience & 45,457 & 38 & 47,750 & 10 & 45,935 & 48 \\
\hline \multirow[t]{4}{*}{ Cataloging: } & Over 14 years experience & 59,206 & 41 & 65,498 & 12 & 60,631 & 53 \\
\hline & 10 to 14 years experience & $\dagger$ & 10 & $\dagger$ & 2 & 58,709 & 12 \\
\hline & 5 to 9 years experience & 50,729 & 17 & 52,093 & 10 & 51,234 & 27 \\
\hline & Under 5 years experience & 46,145 & 22 & 51,065 & 9 & 47,573 & 31 \\
\hline \multirow[t]{4}{*}{ Other: } & Over 14 years experience & 76,990 & 14 & 76,730 & 4 & 76,932 & 18 \\
\hline & 10 to 14 years experience & $\dagger$ & 9 & $\dagger$ & 2 & 58,649 & 11 \\
\hline & 5 to 9 years experience & $\dagger$ & 8 & $\dagger$ & 3 & 53,631 & 11 \\
\hline & Under 5 years experience & $\dagger$ & 26 & $\dagger$ & 2 & 43,770 & 28 \\
\hline \multicolumn{2}{|c|}{ All Positions } & $\$ 61,251$ & 700 & $\$ 62,853$ & 317 & $\$ 61,750$ & 1,017 \\
\hline
\end{tabular}

* Excludes Canadian libraries. Excludes medical and law libraries.

$\dagger$ Salary data are not published when fewer than four individuals are involved in either category.

N/A - No positions were reported in this category. 


\section{TABLE 28: NUMBER AND AVERAGE YEARS OF EXPERIENCE OF MINORITY U.S. ARL UNIVERSITY LIBRARIANS \\ BY POSITION AND SEX, FY 2007-2008*}

\begin{tabular}{|c|c|c|c|c|c|c|c|}
\hline \multirow{2}{*}{\multicolumn{2}{|c|}{ Position }} & \multicolumn{2}{|c|}{ Women } & \multicolumn{2}{|c|}{ Men } & \multicolumn{2}{|c|}{ Total } \\
\hline & & Years & No. & Years & No. & Years & No. \\
\hline \multicolumn{2}{|c|}{ Director } & 29.0 & 3 & 40.0 & 2 & 33.4 & 5 \\
\hline \multicolumn{2}{|c|}{ Associate Director } & 24.5 & 17 & 27.5 & 4 & 25.0 & 21 \\
\hline \multicolumn{2}{|c|}{ Assistant Director } & 23.7 & 3 & 20.0 & 2 & 22.2 & 5 \\
\hline \multicolumn{2}{|c|}{ Head, Branch } & 22.2 & 40 & 17.3 & 12 & 21.1 & 52 \\
\hline \multicolumn{2}{|c|}{ Functional Specialist } & 13.4 & 148 & 10.5 & 120 & 12.1 & 268 \\
\hline \multicolumn{2}{|c|}{ Subject Specialist } & 14.0 & 140 & 12.3 & 53 & 13.5 & 193 \\
\hline \multirow[t]{9}{*}{ Dept. Head: } & Acquisitions & 21.6 & 5 & 24.0 & 2 & 22.3 & 7 \\
\hline & Reference & 21.2 & 5 & 27.0 & 1 & 22.2 & 6 \\
\hline & Cataloging & 21.7 & 10 & 38.0 & 1 & 23.2 & 11 \\
\hline & Serials & 10.3 & 3 & N/A & N/A & 10.3 & 3 \\
\hline & Documents/Maps & 12.2 & 5 & 7.0 & 2 & 10.7 & 7 \\
\hline & Circulation & 22.6 & 7 & 15.7 & 6 & 19.4 & 13 \\
\hline & Rare Books/Manuscripts & 15.3 & 6 & 15.0 & 1 & 15.3 & 7 \\
\hline & Computer Systems & 21.0 & 2 & 17.6 & 10 & 18.2 & 12 \\
\hline & Other & 19.7 & 32 & 19.8 & 14 & 19.7 & 46 \\
\hline \multicolumn{2}{|c|}{ Public Services } & 6.7 & 22 & 14.8 & 4 & 7.9 & 26 \\
\hline \multicolumn{2}{|c|}{ Technical Services } & 13.1 & 19 & 6.0 & 2 & 12.4 & 21 \\
\hline \multicolumn{2}{|c|}{ Administrative Services } & 11.9 & 16 & 12.2 & 5 & 12.0 & 21 \\
\hline \multicolumn{2}{|c|}{ Reference } & 13.8 & 127 & 13.6 & 43 & 13.7 & 170 \\
\hline \multicolumn{2}{|l|}{ Cataloger } & 14.7 & 90 & 13.6 & 33 & 14.4 & 123 \\
\hline \multicolumn{2}{|c|}{ All Positions } & 15.0 & 700 & 13.2 & 317 & 14.5 & 1,017 \\
\hline
\end{tabular}

*Excludes Canadian libraries. Excludes medical and law libraries.

N/A - No positions were reported in this category. 


\section{TABLE 29: NUMBER AND AVERAGE SALARIES OF \\ U.S. ARL UNIVERSITY LIBRARIANS \\ BY YEARS OF EXPERIENCE AND SEX, FY 2007-2808*}

\begin{tabular}{|c|c|c|c|c|c|c|c|}
\hline \multirow[b]{2}{*}{ Experience } & \multicolumn{2}{|c|}{ Women } & \multicolumn{2}{|c|}{ Men } & \multicolumn{2}{|c|}{ Total } & \multirow{2}{*}{$\begin{array}{l}\% \text { of } \\
\text { Total }\end{array}$} \\
\hline & Salary & No. & Salary & No. & Salary & No. & \\
\hline $0-3$ years & $\$ 46,815$ & 578 & $\$ 49,383$ & 301 & $\$ 47,694$ & 879 & $12 \%$ \\
\hline $4-7$ years & 52,127 & 610 & 55,001 & 427 & 53,311 & 1,037 & $14 \%$ \\
\hline $8-11$ years & 58,193 & 621 & 61,395 & 392 & 59,432 & 1,013 & $14 \%$ \\
\hline $12-15$ years & 62,472 & 500 & 66,032 & 304 & 63,818 & 804 & $11 \%$ \\
\hline $16-19$ years & 66,846 & 490 & 68,659 & 294 & 67,526 & 784 & $10 \%$ \\
\hline $20-23$ years & 71,257 & 461 & 74,781 & 251 & 72,499 & 712 & $10 \%$ \\
\hline $24-27$ years & 75,228 & 427 & 80,193 & 235 & 76,991 & 662 & $9 \%$ \\
\hline $28-31$ years & 78,977 & 388 & 86,427 & 236 & 81,795 & 624 & $8 \%$ \\
\hline $32-35$ years & 86,776 & 339 & 88,533 & 223 & 87,473 & 562 & $8 \%$ \\
\hline over 35 years & 85,055 & 275 & 93,868 & 130 & 87,884 & 405 & $5 \%$ \\
\hline All Positions & $\$ 65,559$ & 4,689 & $\$ 68,970$ & 2,793 & $\$ 66,833$ & 7,482 & $100 \%$ \\
\hline
\end{tabular}

*Excludes Canadian libraries. Excludes medical and law libraries.

TABLE 30: NUMBER AND AVERAGE SALARIES OF MINORITY

U.S. ARL UNIVERSITY LIBRARIANS

BY YEARS OF EXPERIENCE AND SEX, FY 2007-2008*

\begin{tabular}{|c|c|c|c|c|c|c|c|}
\hline \multirow[b]{2}{*}{ Experience } & \multicolumn{2}{|c|}{ Women } & \multicolumn{2}{|c|}{ Men } & \multicolumn{2}{|c|}{ Total } & \multirow{2}{*}{$\begin{array}{l}\% \text { of } \\
\text { Total }\end{array}$} \\
\hline & Salary & No. & Salary & No. & Salary & No. & \\
\hline $0-3$ years & $\$ 46,785$ & 117 & $\$ 48,594$ & 53 & $\$ 47,349$ & 170 & $17 \%$ \\
\hline $4-7$ years & 50,673 & 108 & 54,704 & 72 & 52,286 & 180 & $18 \%$ \\
\hline $8-11$ years & 57,335 & 83 & 59,963 & 59 & 58,427 & 142 & $14 \%$ \\
\hline $12-15$ years & 59,957 & 84 & 68,774 & 26 & 62,041 & 110 & $11 \%$ \\
\hline $16-19$ years & 68,556 & 78 & 69,060 & 32 & 68,703 & 110 & $11 \%$ \\
\hline $20-23$ years & 70,163 & 73 & 74,415 & 16 & 70,928 & 89 & $9 \%$ \\
\hline $24-27$ years & 66,115 & 40 & 72,455 & 20 & 68,228 & 60 & $6 \%$ \\
\hline $28-31$ years & 72,127 & 46 & 86,409 & 12 & 75,082 & 58 & $6 \%$ \\
\hline $32-35$ years & 86,403 & 36 & 72,111 & 11 & 83,058 & 47 & $5 \%$ \\
\hline over 35 years & 74,040 & 35 & 87,775 & 16 & 78,349 & 51 & $5 \%$ \\
\hline All Positions & $\$ 61,251$ & 700 & $\$ 62,853$ & 317 & $\$ 61,750$ & 1,017 & $100 \%$ \\
\hline
\end{tabular}

*Excludes Canadian libraries. Excludes medical and law libraries. 

CANADIAN ARL UNIVERSITY LIBRARIES

Tables 31-34 
TABLE 31: FILLED POSITIONS; AVERAGE, MEDIAN, AND BEGINNING PROFESSIONAL SALARIES; AND AVERAGE YEARS OF PROFESSIONAL EXPERIENCE

IN CANADIAN ARL UNIVERSITY LIBRARIES, FY 2007-08*

\begin{tabular}{|c|c|c|c|c|c|c|c|c|}
\hline \multirow[b]{2}{*}{ Institution } & \multirow{2}{*}{$\begin{array}{c}\text { Filled } \\
\text { Positions } \\
\text { FY } 2008\end{array}$} & \multicolumn{2}{|c|}{ Average Salaries } & \multicolumn{2}{|c|}{ Median Salaries } & \multicolumn{2}{|c|}{ Beginning Salaries } & \multirow{2}{*}{$\begin{array}{l}\text { Average } \\
\text { Yrs. Exp. } \\
\text { FY } 2008\end{array}$} \\
\hline & & FY 2007 & FY 2008 & FY2007 & FY2008 & FY2007 & FY2008 & \\
\hline Alberta * & 67 & $\$ 80,505$ & $\$ 84,681$ & $\$ 89,210$ & $\$ 93,456$ & $\$ 47,038$ & 48,685 & 17.3 \\
\hline British Columbia ${ }^{*}$ & 83 & 74,244 & 78,598 & 73,190 & 77,831 & 50,839 & 51,841 & 15.3 \\
\hline Guelph * & 48 & 72,489 & 70,281 & 68,416 & 65,980 & 43,695 & 43,695 & 17.2 \\
\hline Laval & 61 & 62,928 & 67,519 & 64,953 & 71,012 & 42,683 & 45,320 & 16.6 \\
\hline McGill * & 53 & 74,312 & 74,744 & 77,013 & 73,291 & 46,000 & 46,000 & 18.5 \\
\hline McMaster * & 22 & 71,409 & 76,486 & 70,987 & 78,325 & 42,226 & 43,704 & 18.9 \\
\hline Manitoba * & 45 & 84,050 & 87,483 & 87,001 & 89,632 & 45,158 & 46,799 & 23.8 \\
\hline Montreal ${ }^{\ddagger}$ & 85 & 66,179 & 68,235 & 63,214 & 65,918 & 40,124 & 40,926 & 14.9 \\
\hline Queen's * & 35 & 78,481 & 81,829 & 79,890 & 83,878 & 44,715 & 46,079 & 18.7 \\
\hline Saskatchewan * & 40 & 75,513 & 84,386 & 74,832 & 84,307 & 41,807 & 47,101 & 17.0 \\
\hline Toronto ${ }^{*}$ & 136 & 86,489 & 86,859 & 89,880 & 88,821 & 48,700 & 48,700 & 17.2 \\
\hline Waterloo * & 34 & 75,980 & 77,455 & 77,954 & 79,343 & 45,734 & 47,198 & 18.9 \\
\hline Western Ontario * & 63 & 63,053 & 66,739 & 57,992 & 61,423 & 47,630 & 46,000 & 14.6 \\
\hline York ${ }^{*}$ & 56 & 85,643 & 92,462 & 80,763 & 86,615 & 42,225 & 43,275 & 16.5 \\
\hline
\end{tabular}

* Salaries expressed in Canadian dollars. Directors are included in figures for average years of experience and filled positions, but not in the average and median salary statistics. Excludes Canadian medical and law libraries. See Tables 35 and 42 for statistics related to medical and law library salaries. * See Footnotes. 


\section{TABLE 32: NUMBER AND AVERAGE SALARIES OF \\ CANADIAN ARL UNIVERSITY LIBRARIANS \\ BY POSITION AND SEX, FY 2007-08*}

\begin{tabular}{|c|c|c|c|c|c|c|c|}
\hline \multirow{2}{*}{\multicolumn{2}{|c|}{ Position }} & \multicolumn{2}{|c|}{ Women } & \multicolumn{2}{|c|}{ Men } & \multicolumn{2}{|c|}{ Total } \\
\hline & & Salary & No. & Salary & No. & Salary & No. \\
\hline \multicolumn{2}{|l|}{ Director } & $\$ 157,931$ & 8 & $\$ 158,237$ & 6 & $\$ 158,062$ & 14 \\
\hline \multicolumn{2}{|c|}{ Associate Director } & 114,822 & 21 & 118,380 & 13 & 116,183 & 34 \\
\hline \multicolumn{2}{|c|}{ Assistant Director } & 95,071 & 12 & 95,142 & 10 & 95,103 & 22 \\
\hline \multicolumn{2}{|c|}{ Head, Branch } & 94,259 & 46 & 92,673 & 13 & 93,909 & 59 \\
\hline \multicolumn{2}{|c|}{ Functional Specialist } & 70,261 & 65 & 73,153 & 68 & 71,740 & 133 \\
\hline \multicolumn{2}{|c|}{ Subject Specialist } & 72,774 & 52 & 77,826 & 29 & 74,583 & 81 \\
\hline \multirow[t]{9}{*}{ Dept. Head: } & Acquisitions & 80,815 & 7 & 88,884 & 5 & 84,177 & 12 \\
\hline & Reference & $\dagger$ & 11 & $\dagger$ & 1 & 82,773 & 12 \\
\hline & Cataloging & 90,812 & 10 & N/A & N/A & 90,812 & 10 \\
\hline & Serials & $\dagger$ & 3 & N/A & N/A & $\dagger$ & 3 \\
\hline & Documents/Maps & $\dagger$ & 6 & $\dagger$ & 2 & 95,460 & 8 \\
\hline & Circulation & $\dagger$ & 12 & $\dagger$ & 2 & 78,963 & 14 \\
\hline & Rare Books/Manuscripts & $\dagger$ & 3 & $\dagger$ & 4 & 94,595 & 7 \\
\hline & Computer Systems & $\dagger$ & 3 & $\dagger$ & 4 & 89,191 & 7 \\
\hline & Other & 87,516 & 33 & 87,423 & 14 & 87,488 & 47 \\
\hline \multirow[t]{4}{*}{ Reference: } & Over 14 years experience & 83,969 & 85 & 89,595 & 27 & 85,325 & 112 \\
\hline & 10 to 14 years experience & 73,265 & 15 & 77,054 & 8 & 74,583 & 23 \\
\hline & 5 to 9 years experience & 65,782 & 51 & 65,944 & 15 & 65,819 & 66 \\
\hline & Under 5 years experience & 56,748 & 52 & 57,130 & 19 & 56,850 & 71 \\
\hline \multirow[t]{4}{*}{ Cataloging: } & Over 14 years experience & 84,969 & 23 & 75,892 & 13 & 81,691 & 36 \\
\hline & 10 to 14 years experience & $\dagger$ & 4 & $\dagger$ & 1 & 59,646 & 5 \\
\hline & 5 to 9 years experience & $\dagger$ & 10 & $\dagger$ & 2 & 63,475 & 12 \\
\hline & Under 5 years experience & $\dagger$ & 4 & $\dagger$ & 1 & 67,954 & 5 \\
\hline \multirow[t]{4}{*}{ Other: } & Over 14 years experience & 77,531 & 7 & 81,529 & 5 & 79,197 & 12 \\
\hline & 10 to 14 years experience & $\dagger$ & 2 & $\dagger$ & 4 & 71,017 & 6 \\
\hline & 5 to 9 years experience & $\dagger$ & 10 & $\dagger$ & 1 & 61,658 & 11 \\
\hline & Under 5 years experience & $\dagger$ & 4 & $\dagger$ & 2 & 63,499 & 6 \\
\hline \multicolumn{2}{|c|}{ All Positions } & $\$ 79,352$ & 559 & $\$ 81,397$ & 269 & $\$ 80,016$ & 828 \\
\hline
\end{tabular}

* Excludes Canadian medical and law libraries. See Tables 39 and 46 for salaries in medical and law libraries. Salaries expressed in Canadian dollars.

$\dagger$ Salary data are not published when fewer than four individuals are involved in either category.

N/A - No positions were reported in this category. 


\section{TABLE 33: NUMBER AND AVERAGE YEARS OF EXPERIENCE OF CANADIAN ARL UNIVERSITY LIBRARIANS \\ BY POSITION AND SEX, FY 2007-08*}

\begin{tabular}{|c|c|c|c|c|c|c|c|}
\hline \multirow{2}{*}{\multicolumn{2}{|c|}{ Position }} & \multicolumn{2}{|c|}{ Women } & \multicolumn{2}{|c|}{ Men } & \multicolumn{2}{|c|}{ Total } \\
\hline & & Years & No. & Years & No. & Years & No. \\
\hline \multicolumn{2}{|c|}{ Director } & 32.5 & 8 & 24.2 & 6 & 28.9 & 14 \\
\hline \multicolumn{2}{|c|}{ Associate Director } & 25.0 & 21 & 21.8 & 13 & 23.8 & 34 \\
\hline \multicolumn{2}{|c|}{ Assistant Director } & 19.4 & 12 & 21.3 & 10 & 20.3 & 22 \\
\hline \multicolumn{2}{|c|}{ Head, Branch } & 22.5 & 46 & 22.9 & 13 & 22.6 & 59 \\
\hline \multicolumn{2}{|c|}{ Functional Specialist } & 13.8 & 65 & 15.4 & 68 & 14.6 & 133 \\
\hline \multicolumn{2}{|c|}{ Subject Specialist } & 16.4 & 52 & 18.1 & 29 & 17.0 & 81 \\
\hline \multirow[t]{9}{*}{ Dept. Head: } & Acquisitions & 21.7 & 7 & 17.4 & 5 & 19.9 & 12 \\
\hline & Reference & 17.0 & 11 & 30.0 & 1 & 18.1 & 12 \\
\hline & Cataloging & 26.0 & 10 & N/A & N/A & 26.0 & 10 \\
\hline & Serials & 23.7 & 3 & N/A & N/A & 23.7 & 3 \\
\hline & Documents/Maps & 28.2 & 6 & 15.0 & 2 & 24.9 & 8 \\
\hline & Circulation & 20.5 & 12 & 12.5 & 2 & 19.4 & 14 \\
\hline & Rare Books/Manuscripts & 28.0 & 3 & 26.5 & 4 & 27.1 & 7 \\
\hline & Computer Systems & 26.0 & 3 & 18.8 & 4 & 21.9 & 7 \\
\hline & Other & 19.9 & 33 & 15.9 & 14 & 18.7 & 47 \\
\hline \multicolumn{2}{|c|}{ Public Services } & 5.2 & 5 & 10.5 & 4 & 7.6 & 9 \\
\hline \multicolumn{2}{|c|}{ Technical Services } & 12.1 & 7 & 20.0 & 6 & 15.8 & 13 \\
\hline \multicolumn{2}{|c|}{ Administrations } & 16.1 & 11 & 8.0 & 2 & 14.8 & 13 \\
\hline \multicolumn{2}{|c|}{ Reference } & 13.7 & 203 & 13.8 & 69 & 13.7 & 272 \\
\hline \multicolumn{2}{|l|}{ Cataloger } & 19.0 & 41 & 23.4 & 17 & 20.3 & 58 \\
\hline \multicolumn{2}{|c|}{ All Positions } & 17.1 & 559 & 17.2 & 269 & 17.1 & 828 \\
\hline
\end{tabular}

* Excludes Canadian medical and law libraries. See Tables 40 and 47 for figures in medical and law libraries. N/A - No positions were reported in this category. 


\section{TABLE 34: NUMBER AND AVERAGE SALARIES OF \\ CANADIAN ARL UNIVERSITY LIBRARIANS \\ BY YEARS OF EXPERIENCE AND SEX, FY 2007-08*}

\begin{tabular}{|c|c|c|c|c|c|c|c|}
\hline \multirow[b]{2}{*}{ Experience } & \multicolumn{2}{|c|}{ Women } & \multicolumn{2}{|c|}{ Men } & \multicolumn{2}{|c|}{ Total } & \multirow{2}{*}{$\begin{array}{l}\% \text { of } \\
\text { Total }\end{array}$} \\
\hline & Salary & No. & Salary & No. & Salary & No. & \\
\hline $0-3$ years & $\$ 56,729$ & 66 & $\$ 61,596$ & 24 & $\$ 58,027$ & 90 & $11 \%$ \\
\hline $4-7$ years & 63,168 & 92 & 63,438 & 40 & 63,250 & 132 & $16 \%$ \\
\hline $8-11$ years & 69,635 & 62 & 71,068 & 34 & 70,142 & 96 & $12 \%$ \\
\hline $12-15$ years & 77,099 & 46 & 78,813 & 31 & 77,789 & 77 & $9 \%$ \\
\hline $16-19$ years & 80,850 & 62 & 88,277 & 32 & 83,378 & 94 & $11 \%$ \\
\hline $20-23$ years & 90,596 & 65 & 96,369 & 30 & 92,419 & 95 & $11 \%$ \\
\hline $24-27$ years & 93,701 & 43 & 89,187 & 22 & 92,173 & 65 & $8 \%$ \\
\hline $28-31$ years & 91,837 & 51 & 91,684 & 21 & 91,792 & 72 & $9 \%$ \\
\hline $32-35$ years & 94,817 & 39 & 100,998 & 20 & 96,913 & 59 & $7 \%$ \\
\hline over 35 years & 109,880 & 33 & 93,140 & 15 & 104,649 & 48 & $6 \%$ \\
\hline All Positions & $\$ 79,352$ & 559 & $\$ 81,397$ & 269 & $\$ 80,016$ & 828 & $100 \%$ \\
\hline
\end{tabular}

* Excludes Canadian medical and law libraries. See Tables 41 and 48 for salaries in medical and law libraries. Salaries expressed in Canadian dollars. 

ARL UNIVERSITY MEDICAL LIBRARIES

Tables 35-41 
TABLE 35: FILLED POSITIONS; AVERAGE, MEDIAN, BEGINNING PROFESSIONAL SALARIES; AND AVERAGE YEARS OF PROFESSIONAL EXPERIENCE ARL UNIVERSITY MEDICAL LIBRARIES, FY 2007-2008*

\begin{tabular}{|c|c|c|c|c|c|}
\hline Institution & $\begin{array}{c}\text { Filled } \\
\text { Positions }\end{array}$ & $\begin{array}{c}\text { Average } \\
\text { Salary }\end{array}$ & $\begin{array}{c}\text { Median } \\
\text { Salary }\end{array}$ & $\begin{array}{c}\text { Beginning } \\
\text { Salary } \\
\end{array}$ & $\begin{array}{l}\text { Average } \\
\text { Yrs. Exp. }\end{array}$ \\
\hline Alabama & 3 & $\ddagger$ & $\ddagger$ & $\$ 35,000$ & 9.7 \\
\hline Alberta & 5 & $\$ 80,405$ & $\$ 81,218$ & 42,997 & 19.2 \\
\hline Arizona & 15 & 64,593 & 60,212 & 47,325 & 19.7 \\
\hline Boston University & 9 & 53,950 & 52,750 & 40,000 & 12.8 \\
\hline British Columbia & 12 & 65,688 & 68,856 & 45,784 & 16.7 \\
\hline California, Davis & 9 & 75,108 & 79,968 & 40,008 & 25.4 \\
\hline California, Irvine & 2 & 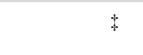 & ‡ & 40,008 & 19.5 \\
\hline California, Los Angeles & 18 & 69,335 & 65,148 & 40,008 & 14.9 \\
\hline California, San Diego & 10 & 61,857 & 62,556 & 40,008 & 13.4 \\
\hline Case Western Reserve & 8 & 58,716 & 61,880 & 35,000 & 25.5 \\
\hline Cincinnati & 19 & 62,743 & 59,998 & 38,000 & 23.8 \\
\hline Columbia & 8 & 59,248 & 56,500 & 51,500 & 18.0 \\
\hline Connecticut & 14 & 73,588 & 70,081 & 53,604 & 16.4 \\
\hline Cornell & 10 & 74,086 & 73,619 & 50,000 & 20.4 \\
\hline Dartmouth & 10 & 58,868 & 58,371 & 40,000 & 17.1 \\
\hline Duke & 21 & 58,648 & 55,821 & 43,000 & 11.8 \\
\hline Emory & 10 & 65,749 & 67,539 & 36,000 & 24.0 \\
\hline Florida & 15 & 52,735 & 48,271 & 42,000 & 7.9 \\
\hline Florida State & 4 & $\neq$ & ‡ & 38,000 & 22.3 \\
\hline George Washington & 14 & 59,824 & 59,930 & 42,000 & 14.4 \\
\hline Georgetown & 9 & 57,773 & 60,207 & 43,000 & 12.2 \\
\hline Harvard & 28 & 70,110 & 67,600 & 47,100 & 10.8 \\
\hline Hawaii & 5 & 46,941 & 44,725 & 42,026 & 16.2 \\
\hline Howard & 6 & 51,311 & 47,802 & 40,000 & 21.5 \\
\hline Illinois, Chicago & 20 & 57,264 & 57,309 & 47,000 & 12.6 \\
\hline Iowa & 11 & 55,303 & 50,501 & 40,000 & 15.5 \\
\hline Johns Hopkins & 24 & 65,497 & 59,467 & 48,000 & 16.5 \\
\hline Kansas & 8 & 50,936 & 46,800 & 38,000 & 13.9 \\
\hline Kentucky & 12 & 57,724 & 58,444 & 38,500 & 22.8 \\
\hline Louisiana State & 1 & $\ddagger$ & $\ddagger$ & 36,000 & 2.0 \\
\hline Louisville & 8 & 54,615 & 50,994 & 37,000 & 20.9 \\
\hline McGill & 9 & 65,033 & 62,033 & 40,625 & 19.4 \\
\hline McMaster & 9 & 60,782 & 60,057 & 38,598 & 17.0 \\
\hline Manitoba & 17 & 60,909 & 57,099 & 41,331 & 15.0 \\
\hline Miami & 11 & 68,121 & 67,174 & 44,000 & 23.5 \\
\hline Michigan & 17 & 58,387 & 58,168 & 41,000 & 15.8 \\
\hline Minnesota & 14 & 58,657 & 59,756 & 40,000 & 17.1 \\
\hline Missouri & 10 & 45,421 & 43,000 & 38,500 & 16.1 \\
\hline Montreal ${ }^{\S}$ & 7 & 60,540 & 58,216 & 44,630 & 17.3 \\
\hline
\end{tabular}




\section{TABLE 35: FILLED POSITIONS; AVERAGE, MEDIAN, BEGINNING PROFESSIONAL SALARIES; AND AVERAGE YEARS OF PROFESSIONAL EXPERIENCE ARL UNIVERSITY MEDICAL LIBRARIES, FY 2007-2008*}

\begin{tabular}{|c|c|c|c|c|c|}
\hline Institution & $\begin{array}{c}\text { Filled } \\
\text { Positions }\end{array}$ & $\begin{array}{c}\text { Average } \\
\text { Salary }\end{array}$ & $\begin{array}{c}\text { Median } \\
\text { Salary }\end{array}$ & $\begin{array}{c}\text { Beginning } \\
\text { Salary }\end{array}$ & $\begin{array}{l}\text { Average } \\
\text { Yrs. Exp. }\end{array}$ \\
\hline Nebraska & 12 & $\$ 61,800$ & $\$ 55,700$ & $\$ 40,000$ & 20.3 \\
\hline New Mexico & 20 & 77,298 & 68,855 & 38,600 & 19.2 \\
\hline New York University & 29 & 64,933 & 59,232 & 55,000 & 12.1 \\
\hline North Carolina & 28 & 66,519 & 64,506 & 45,000 & 21.3 \\
\hline Northwestern & 16 & 55,138 & 51,607 & 40,000 & 14.8 \\
\hline Ohio State & 14 & 57,918 & 58,634 & 42,000 & 15.2 \\
\hline Oklahoma & 6 & 56,425 & 57,228 & 35,000 & 24.0 \\
\hline Oklahoma State & 3 & $\ddagger$ & $\ddagger$ & 38,000 & 25.3 \\
\hline Pennsylvania & 12 & 61,476 & 59,911 & 42,500 & 15.8 \\
\hline Pennsylvania State & 5 & 57,390 & 60,012 & 41,200 & 15.2 \\
\hline Pittsburgh & 26 & 55,391 & 53,814 & 40,000 & 13.7 \\
\hline Queen`s & 9 & 62,816 & 60,843 & 40,695 & 13.9 \\
\hline Rochester & 23 & 53,978 & 50,197 & 36,700 & 19.0 \\
\hline Saskatchewan & 7 & 53,670 & 50,746 & 41,598 & 6.9 \\
\hline South Carolina & 8 & 47,326 & 43,466 & 34,500 & 10.8 \\
\hline Southern California & 14 & 72,375 & 73,560 & 45,600 & 20.4 \\
\hline Southern Illinois & 6 & 58,937 & 63,440 & 41,000 & 22.5 \\
\hline SUNY Buffalo & 15 & 59,971 & 58,842 & 45,000 & 19.2 \\
\hline SUNY Stony Brook & 20 & 59,782 & 57,702 & 45,000 & 12.6 \\
\hline Temple & 10 & 55,785 & 55,731 & 39,000 & 17.5 \\
\hline Tennessee, Knoxville & 4 & $\ddagger$ & ‡ & 38,000 & 16.5 \\
\hline Tennessee, Memphis & 10 & 57,325 & 55,371 & 40,000 & 19.3 \\
\hline Texas A\&M & 13 & 55,515 & 52,836 & 44,000 & 15.4 \\
\hline Texas Tech & 20 & 49,088 & 46,167 & 35,000 & 21.3 \\
\hline Toronto $\$$ & 18 & 71,938 & 68,161 & 43,010 & 17.8 \\
\hline Tulane & 7 & 42,862 & 38,268 & 37,000 & 15.4 \\
\hline Utah & 13 & 55,692 & 56,313 & 35,000 & 15.6 \\
\hline Vanderbilt & 28 & 55,562 & 57,150 & 39,000 & 10.7 \\
\hline Virginia & 14 & 63,268 & 63,800 & 45,000 & 18.9 \\
\hline Washington & 18 & 61,802 & 57,600 & 41,000 & 22.7 \\
\hline Washington U.-St. Louis & 19 & 62,676 & 51,825 & 40,000 & 24.5 \\
\hline Wayne State & 11 & 48,089 & 44,863 & 45,000 & 24.6 \\
\hline Wisconsin & 18 & 54,215 & 52,978 & 39,338 & 11.1 \\
\hline Yale & 19 & 72,169 & 70,354 & 49,500 & 15.7 \\
\hline
\end{tabular}




\section{TABLE 36: BEGINNING PROFESSIONAL SALARIES IN ARL UNIVERSITY MEDICAL LIBRARIES \\ RANK ORDER TABLE, FY 2007-2008*}

\begin{tabular}{|c|c|c|c|c|c|}
\hline Rank & Institution & Salary & Rank & Institution & Salary \\
\hline 1 & New York & $\$ 55,000$ & 37 & Calif. Irvine & 40,008 \\
\hline 2 & Connecticut & 53,604 & 37 & Calif. Los Angeles & 40,008 \\
\hline 3 & Columbia & 51,500 & 37 & Calif. San Diego & 40,008 \\
\hline 4 & Cornell & 50,000 & 41 & Boston University & 40,000 \\
\hline 5 & Yale & 49,500 & 41 & Dartmouth & 40,000 \\
\hline 6 & Johns Hopkins & 48,000 & 41 & Howard & 40,000 \\
\hline 7 & Arizona & 47,325 & 41 & Iowa & 40,000 \\
\hline 8 & Harvard & 47,100 & 41 & Minnesota & 40,000 \\
\hline 9 & Illinois, Chicago & 47,000 & 41 & Nebraska & 40,000 \\
\hline 10 & British Columbia & 45,784 & 41 & Northwestern & 40,000 \\
\hline 11 & Southern California & 45,600 & 41 & Pittsburgh & 40,000 \\
\hline 12 & North Carolina & 45,000 & 41 & Tennessee, Memphis Medical & 40,000 \\
\hline 12 & SUNY Buffalo & 45,000 & 41 & Washington - St. Louis & 40,000 \\
\hline 12 & SUNY Stony Brook & 45,000 & 51 & Wisconsin & 39,338 \\
\hline 12 & Virginia & 45,000 & 52 & Temple & 39,000 \\
\hline 12 & Wayne State & 45,000 & 52 & Vanderbilt & 39,000 \\
\hline 17 & Montreal & 44,630 & 54 & New Mexico & 38,600 \\
\hline 18 & Miami & 44,000 & 55 & McMaster & 38,598 \\
\hline 18 & Texas A\&M & 44,000 & 56 & Kentucky & 38,500 \\
\hline 20 & Toronto & 43,010 & 56 & Missouri & 38,500 \\
\hline 21 & Duke & 43,000 & 58 & Cincinnati & 38,000 \\
\hline 21 & Georgetown & 43,000 & 58 & Florida State & 38,000 \\
\hline 23 & Alberta & 42,997 & 58 & Kansas & 38,000 \\
\hline 24 & Pennsylvania & 42,500 & 58 & Oklahoma State & 38,000 \\
\hline 25 & Hawaii & 42,026 & 58 & Tennessee, Knoxville & 38,000 \\
\hline 26 & Florida & 42,000 & 63 & Louisville & 37,000 \\
\hline 26 & George Washington & 42,000 & 63 & Tulane & 37,000 \\
\hline 26 & Ohio State & 42,000 & 65 & Rochester & 36,700 \\
\hline 29 & Saskatchewan & 41,598 & 66 & Emory & 36,000 \\
\hline 30 & Manitoba & 41,331 & 66 & Louisiana State & 36,000 \\
\hline 31 & Pennsylvania State & 41,200 & 68 & Alabama & 35,000 \\
\hline 32 & Michigan & 41,000 & 68 & Case Western Reserve & 35,000 \\
\hline 32 & Southern Illinois & 41,000 & 68 & Oklahoma & 35,000 \\
\hline 32 & Washington & 41,000 & 68 & Texas Tech & 35,000 \\
\hline 35 & Queen's & 40,695 & 68 & Utah & 35,000 \\
\hline 36 & McGill & 40,625 & 73 & South Carolina & 34,500 \\
\hline 37 & Calif. Davis & 40,008 & & & \\
\hline
\end{tabular}

* Beginning salary figures represent officially designated base, not necessarily salaries of actual incumbents.

${ }^{\dagger}$ Canadian salaries expressed in U.S. dollars. 


\section{TABLE 37: MEDIAN PROFESSIONAL SALARIES \\ IN ARL UNIVERSITY MEDICAL LIBRARIES \\ RANK ORDER TABLE, FY 2007-2008*}

\begin{tabular}{|c|c|c|c|c|c|}
\hline Rank & Institution & Salary & Rank & Institution & Salary \\
\hline 1 & Alberta & $\$ 81,218$ & 35 & Montreal & $\$ 58,216$ \\
\hline 2 & California, Davis & 79,968 & 36 & Michigan & 58,168 \\
\hline 3 & Cornell & 73,619 & 37 & SUNY Stony Brook & 57,702 \\
\hline 4 & Southern California & 73,560 & 38 & Washington & 57,600 \\
\hline 5 & Yale & 70,354 & 39 & Illinois, Chicago & 57,309 \\
\hline 6 & Connecticut & 70,081 & 40 & Oklahoma & 57,228 \\
\hline 7 & British Columbia & 68,856 & 41 & Vanderbilt & 57,150 \\
\hline 8 & New Mexico & 68,855 & 42 & Manitoba & 57,099 \\
\hline 9 & Toronto & 68,161 & 43 & Columbia & 56,500 \\
\hline 10 & Harvard & 67,600 & 44 & Utah & 56,313 \\
\hline 11 & Emory & 67,539 & 45 & Duke & 55,821 \\
\hline 12 & Miami & 67,174 & 46 & Temple & 55,731 \\
\hline 13 & California, Los Angeles & 65,148 & 47 & Nebraska & 55,700 \\
\hline 14 & North Carolina & 64,506 & 48 & Tennessee, Memphis & 55,371 \\
\hline 15 & Virginia & 63,800 & 49 & Pittsburgh & 53,814 \\
\hline 16 & Southern Illinois & 63,440 & 50 & Wisconsin & 52,978 \\
\hline 17 & California, San Diego & 62,556 & 51 & Texas A\&M & 52,836 \\
\hline 18 & McGill & 62,033 & 52 & Boston University & 52,750 \\
\hline 19 & Case Western Reserve & 61,880 & 53 & Washington U.-St. Louis & 51,825 \\
\hline 20 & Queen`s & 60,843 & 54 & Northwestern & 51,607 \\
\hline 21 & Arizona & 60,212 & 55 & Louisville & 50,994 \\
\hline 22 & Georgetown & 60,207 & 56 & Saskatchewan & 50,746 \\
\hline 23 & McMaster & 60,057 & 57 & Iowa & 50,501 \\
\hline 24 & Pennsylvania State & 60,012 & 58 & Rochester & 50,197 \\
\hline 25 & Cincinnati & 59,998 & 59 & Florida & 48,271 \\
\hline 26 & George Washington & 59,930 & 60 & Howard & 47,802 \\
\hline 27 & Pennsylvania & 59,911 & 61 & Kansas & 46,800 \\
\hline 28 & Minnesota & 59,756 & 62 & Texas Tech & 46,167 \\
\hline 29 & Johns Hopkins & 59,467 & 63 & Wayne State & 44,863 \\
\hline 30 & New York University & 59,232 & 64 & Hawaii & 44,725 \\
\hline 31 & SUNY Buffalo & 58,842 & 65 & South Carolina & 43,466 \\
\hline 32 & Ohio State & 58,634 & 66 & Missouri & 43,000 \\
\hline 33 & Kentucky & 58,444 & 67 & Tulane & 38,268 \\
\hline 34 & Dartmouth & 58,371 & & & \\
\hline
\end{tabular}

* Salaries of directors are not included in the calculation of medians. Alabama, California-Irvine, Florida State, Louisiana State, Oklahoma State, and Tennessee-Knoxville are not ranked because they reported fewer than four individuals.

$\dagger$ Canadian salaries expressed in U.S. dollars. 


\section{TABLE 38: AVERAGE PROFESSIONAL SALARIES IN ARL UNIVERSITY MEDICAL LIBRARIES \\ RANK ORDER TABLE, FY 2007-2008*}

\begin{tabular}{|c|c|c|c|c|c|}
\hline Rank & Institution & Salary & Rank & Institution & Salary \\
\hline 1 & Alberta & $\$ 80,405$ & 35 & Dartmouth & $\$ 58,868$ \\
\hline 2 & New Mexico & 77,298 & 36 & Case Western Reserve & 58,716 \\
\hline 3 & California, Davis & 75,108 & 37 & Minnesota & 58,657 \\
\hline 4 & Cornell & 74,086 & 38 & Duke & 58,648 \\
\hline 5 & Connecticut & 73,588 & 39 & Michigan & 58,387 \\
\hline 6 & Southern California & 72,375 & 40 & Ohio State & 57,918 \\
\hline 7 & Yale & 72,169 & 41 & Georgetown & 57,773 \\
\hline 8 & Toronto & 71,938 & 42 & Kentucky & 57,724 \\
\hline 9 & Harvard & 70,110 & 43 & Pennsylvania State & 57,390 \\
\hline 10 & California, Los Angeles & 69,335 & 44 & Tennessee, Memphis & 57,325 \\
\hline 11 & Miami & 68,121 & 45 & Illinois, Chicago & 57,264 \\
\hline 12 & North Carolina & 66,519 & 46 & Oklahoma & 56,425 \\
\hline 13 & Emory & 65,749 & 47 & Temple & 55,785 \\
\hline 14 & British Columbia & 65,688 & 48 & Utah & 55,692 \\
\hline 15 & Johns Hopkins & 65,497 & 49 & Vanderbilt & 55,562 \\
\hline 16 & McGill & 65,033 & 50 & Texas A\&M & 55,515 \\
\hline 17 & New York University & 64,933 & 51 & Pittsburgh & 55,391 \\
\hline 18 & Arizona & 64,593 & 52 & Iowa & 55,303 \\
\hline 19 & Virginia & 63,268 & 53 & Northwestern & 55,138 \\
\hline 20 & Queen`s & 62,816 & 54 & Louisville & 54,615 \\
\hline 21 & Cincinnati & 62,743 & 55 & Wisconsin & 54,215 \\
\hline 22 & Washington U.-St .Louis & 62,676 & 56 & Rochester & 53,978 \\
\hline 23 & California, San Diego & 61,857 & 57 & Boston University & 53,950 \\
\hline 24 & Washington & 61,802 & 58 & Saskatchewan & 53,670 \\
\hline 25 & Nebraska & 61,800 & 59 & Florida & 52,735 \\
\hline 26 & Pennsylvania & 61,476 & 60 & Howard & 51,311 \\
\hline 27 & Manitoba & 60,909 & 61 & Kansas & 50,936 \\
\hline 28 & McMaster & 60,782 & 62 & Texas Tech & 49,088 \\
\hline 29 & Montreal & 60,540 & 63 & Wayne State & 48,089 \\
\hline 30 & SUNY Buffalo & 59,971 & 64 & South Carolina & 47,326 \\
\hline 31 & George Washington & 59,824 & 65 & Hawaii & 46,941 \\
\hline 32 & SUNY Stony Brook & 59,782 & 66 & Missouri & 45,421 \\
\hline 33 & Columbia & 59,248 & 67 & Tulane & 42,862 \\
\hline 34 & Southern Illinois & 58,937 & & & \\
\hline
\end{tabular}

* Salaries of directors are not included in the calculation of averages. Alabama, California-Irvine, Florida State, Louisiana State, Oklahoma State, and Tennessee-Knoxville are not ranked because they reported fewer than four individuals.

${ }^{\dagger}$ Canadian salaries expressed in U.S. dollars. 


\section{TABLE 39: NUMBER AND AVERAGE SALARIES OF ARL UNIVERSITY MEDICAL LIBRARIANS \\ BY POSITION AND SEX, FY 2007-2008}

\begin{tabular}{|c|c|c|c|c|c|c|c|}
\hline \multirow{2}{*}{\multicolumn{2}{|c|}{ Position }} & \multicolumn{2}{|c|}{ Women } & \multicolumn{2}{|c|}{ Men } & \multicolumn{2}{|c|}{ Total } \\
\hline & & Salary & No. & Salary & No. & Salary & No. \\
\hline \multicolumn{2}{|c|}{ Head, Medical } & $\$ 83,530$ & 35 & $\$ 88,216$ & 12 & $\$ 84,726$ & 47 \\
\hline \multicolumn{2}{|c|}{ Associate Director } & 65,047 & 41 & 60,450 & 6 & 64,460 & 47 \\
\hline \multicolumn{2}{|c|}{ Assistant Director } & 115,586 & 48 & 129,238 & 19 & 119,457 & 67 \\
\hline \multicolumn{2}{|c|}{ Head, Branch } & $\dagger$ & 28 & $\dagger$ & 3 & 64,141 & 31 \\
\hline \multicolumn{2}{|c|}{ Functional Specialist } & 56,667 & 71 & 59,774 & 63 & 58,128 & 134 \\
\hline \multicolumn{2}{|c|}{ Subject Specialist } & 57,793 & 51 & 56,534 & 10 & 57,586 & 61 \\
\hline \multirow[t]{9}{*}{ Dept. Head: } & Acquisitions & $\dagger$ & 15 & $\dagger$ & 3 & 65,044 & 18 \\
\hline & Reference & 66,909 & 15 & 74,848 & 11 & 70,268 & 26 \\
\hline & Cataloging & $\dagger$ & 9 & $\dagger$ & 2 & 64,525 & 11 \\
\hline & Serials & $\dagger$ & 5 & $\dagger$ & 1 & 59,519 & 6 \\
\hline & Documents/Maps & $\dagger$ & 1 & N/A & N/A & $\dagger$ & 1 \\
\hline & Circulation & 62,938 & 9 & 61,430 & 8 & 62,228 & 17 \\
\hline & Rare Books/Manuscripts & 64,145 & 5 & 69,048 & 5 & 66,596 & 10 \\
\hline & Computer Systems & 70,599 & 9 & 87,653 & 7 & 78,060 & 16 \\
\hline & Other & 63,165 & 54 & 80,663 & 13 & 66,560 & 67 \\
\hline \multirow[t]{4}{*}{ Reference: } & Over 14 years experience & 60,373 & 97 & 66,005 & 15 & 61,127 & 112 \\
\hline & 10 to 14 years experience & 58,682 & 20 & 59,289 & 12 & 58,909 & 32 \\
\hline & 5 to 9 years experience & 51,332 & 62 & 61,480 & 10 & 52,741 & 72 \\
\hline & Under 5 years experience & 44,866 & 48 & 44,100 & 13 & 44,703 & 61 \\
\hline \multirow[t]{4}{*}{ Cataloging: } & Over 14 years experience & $\dagger$ & 7 & $\dagger$ & 1 & 57,261 & 8 \\
\hline & 10 to 14 years experience & $\dagger$ & 1 & $\dagger$ & 1 & $\dagger$ & 2 \\
\hline & 5 to 9 years experience & $\dagger$ & 3 & $\dagger$ & 1 & 48,691 & 4 \\
\hline & Under 5 years experience & $\dagger$ & 3 & $\mathrm{~N} / \mathrm{A}$ & N/A & $\dagger$ & 3 \\
\hline \multirow[t]{4}{*}{ Other: } & Over 14 years experience & 65,973 & 32 & 61,176 & 8 & 65,014 & 40 \\
\hline & 10 to 14 years experience & 56,024 & 8 & 53,224 & 5 & 54,947 & 13 \\
\hline & 5 to 9 years experience & $\dagger$ & 13 & $\dagger$ & 2 & 53,503 & 15 \\
\hline & Under 5 years experience & 44,684 & 12 & 48,172 & 4 & 45,556 & 16 \\
\hline \multicolumn{2}{|c|}{ All Positions } & $\$ 63,563$ & 702 & $\$ 69,262$ & 235 & $\$ 64,992$ & 937 \\
\hline
\end{tabular}

* Canadian salaries expressed in U.S. dollars.

${ }^{\dagger}$ Salary data are not published when fewer than four individuals are involved in either category.

N/A - No positions were reported in this category. 


\section{TABLE 40: NUMBER AND AVERAGE YEARS OF EXPERIENCE OF ARL UNIVERSITY MEDICAL LIBRARIANS \\ BY POSITION AND SEX, FY 2007-2008}

\begin{tabular}{|c|c|c|c|c|c|c|c|}
\hline \multirow{2}{*}{\multicolumn{2}{|c|}{ Position }} & \multicolumn{2}{|c|}{ Women } & \multicolumn{2}{|c|}{ Men } & \multicolumn{2}{|c|}{ Total } \\
\hline & & Years & No. & Years & No. & Years & No. \\
\hline \multicolumn{2}{|c|}{ Head, Medical } & 22.3 & 35 & 23.7 & 12 & 22.6 & 47 \\
\hline \multicolumn{2}{|c|}{ Associate Director } & 18.6 & 41 & 9.8 & 6 & 17.5 & 47 \\
\hline \multicolumn{2}{|c|}{ Assistant Director } & 27.3 & 48 & 29.1 & 19 & 27.8 & 67 \\
\hline \multicolumn{2}{|c|}{ Head, Branch } & 18.8 & 28 & 26.3 & 3 & 19.5 & 31 \\
\hline \multicolumn{2}{|c|}{ Functional Specialist } & 14.6 & 71 & 10.5 & 63 & 12.7 & 134 \\
\hline \multicolumn{2}{|c|}{ Subject Specialist } & 15.8 & 51 & 10.4 & 10 & 14.9 & 61 \\
\hline \multirow[t]{9}{*}{ Dept. Head: } & Acquisitions & 18.7 & 15 & 29.7 & 3 & 20.5 & 18 \\
\hline & Reference & 23.5 & 15 & 21.9 & 11 & 22.8 & 26 \\
\hline & Cataloging & 17.7 & 9 & 29.5 & 2 & 19.8 & 11 \\
\hline & Serials & 25.6 & 5 & 30.0 & 1 & 26.3 & 6 \\
\hline & Documents/Maps & 17.0 & 1 & N/A & N/A & 17.0 & 1 \\
\hline & Circulation & 17.0 & 9 & 8.4 & 8 & 12.9 & 17 \\
\hline & Rare Books/Manuscripts & 28.2 & 5 & 24.6 & 5 & 26.4 & 10 \\
\hline & Computer Systems & 15.7 & 9 & 16.7 & 7 & 16.1 & 16 \\
\hline & Other & 20.2 & 54 & 15.5 & 13 & 19.3 & 67 \\
\hline \multicolumn{2}{|c|}{ Public Services } & 16.8 & 38 & 11.6 & 12 & 15.5 & 50 \\
\hline \multicolumn{2}{|c|}{ Technical Services } & 15.8 & 14 & 20.7 & 3 & 16.6 & 17 \\
\hline \multicolumn{2}{|c|}{ Administrative Services } & 17.8 & 13 & 22.0 & 4 & 18.8 & 17 \\
\hline \multicolumn{2}{|c|}{ Reference } & 13.7 & 227 & 12.4 & 50 & 13.5 & 277 \\
\hline \multicolumn{2}{|l|}{ Cataloger } & 15.6 & 14 & 18.7 & 3 & 16.1 & 17 \\
\hline \multicolumn{2}{|c|}{ All Positions } & 17.3 & 702 & 15.5 & 235 & 16.8 & 937 \\
\hline
\end{tabular}

N/A - No positions were reported in this category. 


\section{TABLE 41: NUMBER AND AVERAGE SALARIES OF \\ ARL UNIVERSITY MEDICAL LIBRARIANS \\ BY YEARS OF EXPERIENCE AND SEX, FY 2007-2008*}

\begin{tabular}{lccccccc}
\hline & \multicolumn{2}{c}{ Women } & \multicolumn{2}{c}{ Men } & \multicolumn{2}{c}{ Total } & \% of \\
Experience & Salary & No. & Salary & No. & Salary & No. & Total \\
\hline & & & & & & & \\
$0-3$ years & $\$ 45,929$ & 81 & $\$ 53,207$ & 36 & $\$ 48,168$ & 117 & $12 \%$ \\
$4-7$ years & 52,485 & 99 & 60,330 & 32 & 54,401 & 131 & $14 \%$ \\
$8-11$ years & 58,836 & 89 & 59,212 & 28 & 58,926 & 117 & $12 \%$ \\
$12-15$ years & 62,244 & 71 & 68,832 & 36 & 64,460 & 107 & $11 \%$ \\
$16-19$ years & 61,084 & 58 & 74,428 & 21 & 64,631 & 79 & $8 \%$ \\
$20-23$ years & 65,391 & 79 & 75,523 & 19 & 67,355 & 98 & $10 \%$ \\
$24-27$ years & 70,378 & 69 & 80,285 & 18 & 72,427 & 87 & $9 \%$ \\
$28-31$ years & 75,164 & 73 & 76,689 & 28 & 75,587 & 101 & $11 \%$ \\
$32-35$ years & 89,896 & 43 & 112,976 & 9 & 93,891 & 52 & $6 \%$ \\
over 35 years & 78,293 & 40 & 85,944 & 8 & 79,569 & 48 & $5 \%$ \\
& & & & & & & $\mathbf{9 3 7}$ \\
\hline All Positions & $\mathbf{\$ 6 3 , 5 6 3}$ & $\mathbf{7 0 2}$ & $\mathbf{\$ 6 9 , 2 6 2}$ & $\mathbf{2 3 5}$ & $\mathbf{\$ 6 4 , 9 9 2}$ & $\mathbf{1 0 0 \%}$ \\
\hline
\end{tabular}

* Canadian salaries expressed in U.S. dollars.

$\uparrow$ Salary data are not published when fewer than four individuals are involved in either category. 

ARL UNIVERSITY LAW LIBRARIES

Tables 42-48 
TABLE 42: FILLED POSITIONS; AVERAGE, MEDIAN, BEGINNING PROFESSIONAL

SALARIES; AND AVERAGE YEARS OF EXPERIENCE IN ARL UNIVERSITY LAW LIBRARIES, FY 2007-2008*

\begin{tabular}{|c|c|c|c|c|c|}
\hline Institution & $\begin{array}{c}\text { Filled } \\
\text { Positions }\end{array}$ & $\begin{array}{c}\text { Average } \\
\text { Salary }\end{array}$ & $\begin{array}{c}\text { Median } \\
\text { Salary }\end{array}$ & $\begin{array}{c}\text { Beginning } \\
\text { Salary }\end{array}$ & $\begin{array}{l}\text { Average } \\
\text { Yrs. Exp. }\end{array}$ \\
\hline Alabama & 9 & $\$ 56,059$ & $\$ 57,349$ & $\$ 40,000$ & 19.3 \\
\hline Alberta & 4 & 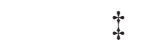 & $t$ & 42,997 & 27.5 \\
\hline Arizona & 11 & 63,672 & 63,854 & 50,000 & 17.6 \\
\hline Arizona State & 9 & 63,087 & 60,966 & 43,000 & 14.3 \\
\hline Boston University & 10 & 64,222 & 61,000 & 55,000 & 19.5 \\
\hline Boston College & 13 & 73,004 & 78,408 & 41,050 & 20.6 \\
\hline British Columbia & 3 & $\ddagger$ & $\ddagger$ & 45,784 & 21.3 \\
\hline California, Davis & 6 & 89,280 & 92,436 & 40,008 & 19.3 \\
\hline California, Los Angeles & 13 & 77,232 & 79,968 & 40,008 & 12.5 \\
\hline Case Western Reserve & 13 & 62,667 & 62,791 & 35,000 & 16.9 \\
\hline Cincinnati & 8 & 57,367 & 50,000 & 40,425 & 20.3 \\
\hline Colorado & 8 & 68,030 & 57,597 & 44,000 & 15.4 \\
\hline Columbia & 15 & 74,710 & 71,016 & 51,500 & 13.5 \\
\hline Connecticut & 11 & 68,743 & 69,788 & 39,263 & 17.0 \\
\hline Cornell & 8 & 73,209 & 68,650 & 54,000 & 15.3 \\
\hline Duke & 10 & 72,106 & 71,900 & 45,000 & 17.7 \\
\hline Emory & 9 & 55,126 & 54,422 & 44,000 & 14.8 \\
\hline Florida & 9 & 57,474 & 53,556 & 42,000 & 19.7 \\
\hline Florida State & 10 & 52,937 & 51,500 & 50,000 & 17.9 \\
\hline George Washington & 20 & 81,629 & 73,266 & 55,000 & 14.2 \\
\hline Georgetown & 23 & 73,964 & 67,950 & 46,500 & 12.0 \\
\hline Georgia & 8 & 53,614 & 50,887 & 42,000 & 15.0 \\
\hline Harvard & 48 & 75,589 & 71,380 & 47,100 & 18.2 \\
\hline Hawaii & 5 & 79,336 & 79,376 & 55,000 & 17.2 \\
\hline Houston & 14 & 52,302 & 48,000 & 50,000 & 13.0 \\
\hline Howard & 8 & 47,556 & 48,460 & 43,779 & 18.5 \\
\hline Illinois, Urbana & 8 & 62,582 & 68,313 & 51,000 & 16.9 \\
\hline Indiana & 9 & 67,432 & 64,176 & 40,000 & 21.1 \\
\hline Iowa & 16 & 70,570 & 66,500 & 40,000 & 20.6 \\
\hline Kansas & 8 & 47,828 & 43,000 & 33,000 & 7.6 \\
\hline Kentucky & 5 & 52,734 & 51,868 & 38,500 & 13.2 \\
\hline Louisiana State & 11 & 61,972 & 53,698 & 40,000 & 17.9 \\
\hline Louisville & 6 & 56,866 & 53,853 & 37,000 & 17.3 \\
\hline McGill & 3 & $t$ & $t$ & 40,625 & 18.0 \\
\hline Manitoba & 3 & $\ddagger$ & $t$ & 41,331 & 28.3 \\
\hline Miami & 16 & 56,240 & 55,432 & 44,000 & 15.8 \\
\hline Michigan & 10 & 77,070 & 71,328 & 47,218 & 18.1 \\
\hline Minnesota & 14 & 72,812 & 65,500 & 42,000 & 18.0 \\
\hline Missouri & 8 & 52,200 & 55,122 & 38,500 & 11.4 \\
\hline
\end{tabular}

76 · ARL Annual Salary Survey 2007-2008 


\section{TABLE 42: FILLED POSITIONS; AVERAGE, MEDIAN, BEGINNING PROFESSIONAL SALARIES; AND AVERAGE YEARS OF EXPERIENCE IN ARL UNIVERSITY LAW LIBRARIES, FY 2007-2008*}

\begin{tabular}{|c|c|c|c|c|c|}
\hline Institution & $\begin{array}{c}\text { Filled } \\
\text { Positions }\end{array}$ & $\begin{array}{c}\text { Average } \\
\text { Salary }\end{array}$ & $\begin{array}{c}\text { Median } \\
\text { Salary }\end{array}$ & $\begin{array}{c}\text { Beginning } \\
\text { Salary }\end{array}$ & $\begin{array}{l}\text { Average } \\
\text { Yrs. Exp. }\end{array}$ \\
\hline Montreal & 4 & $\ddagger$ & $t$ & $\$ 44,630$ & 11.3 \\
\hline Nebraska & 5 & $\$ 58,323$ & $\$ 57,917$ & 35,000 & 15.4 \\
\hline New Mexico & 6 & 69,741 & 75,000 & 48,000 & 11.2 \\
\hline New York University & 19 & 75,491 & 72,546 & 57,566 & 20.1 \\
\hline North Carolina & 12 & 68,749 & 66,010 & 45,000 & 13.2 \\
\hline Northwestern & 14 & 59,235 & 57,684 & 40,000 & 16.5 \\
\hline Notre Dame & 12 & 68,424 & 62,200 & 40,000 & 17.9 \\
\hline Ohio State & 6 & 66,768 & 63,963 & 41,500 & 14.5 \\
\hline Oklahoma & 7 & 52,831 & 47,521 & 39,000 & 12.6 \\
\hline Oregon & 7 & 53,094 & 51,951 & 40,000 & 20.7 \\
\hline Pennsylvania & 14 & 67,577 & 61,815 & 42,000 & 16.8 \\
\hline Pennsylvania State & 7 & 70,980 & 68,760 & 41,200 & 22.7 \\
\hline Queen`s & 2 & $t$ & 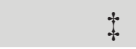 & 40,695 & 11.0 \\
\hline Rutgers, Camden & 9 & 68,312 & 72,991 & 52,000 & 20.8 \\
\hline Rutgers, Newark & 9 & 69,399 & 61,279 & 50,000 & 16.3 \\
\hline Saskatchewan & 3 & $t$ & $t$ & 41,598 & 20.0 \\
\hline South Carolina & 10 & 64,176 & 66,950 & 50,000 & 11.7 \\
\hline Southern Illinois & 5 & 62,040 & 55,146 & 50,000 & 9.8 \\
\hline SUNY Buffalo & 10 & 69,026 & 70,228 & 45,000 & 23.6 \\
\hline Syracuse & 10 & 56,574 & 53,310 & U/A & 14.8 \\
\hline Temple & 9 & 61,754 & 53,043 & 40,800 & 23.4 \\
\hline Tennessee & 10 & 69,284 & 59,440 & 40,000 & 16.4 \\
\hline Texas & 13 & 61,677 & 54,288 & 37,000 & 17.3 \\
\hline Texas Tech & 8 & 61,269 & 59,474 & 45,000 & 13.4 \\
\hline Toronto & 5 & 80,540 & 84,084 & 43,010 & 17.0 \\
\hline Tulane & 6 & 55,909 & 55,310 & 37,000 & 14.8 \\
\hline Utah & 7 & 61,380 & 65,265 & 45,000 & 20.7 \\
\hline Vanderbilt & 6 & 63,567 & 57,976 & 39,000 & 18.3 \\
\hline Virginia & 12 & 62,336 & 60,000 & 52,700 & 14.6 \\
\hline Washington & 16 & 68,048 & 67,956 & 50,000 & 22.3 \\
\hline Washington U.-St. Louis & 1 & $t$ & $\ddagger$ & 49,000 & 26.0 \\
\hline Wayne State & 9 & 44,943 & 43,450 & 45,000 & 21.2 \\
\hline Western Ontario & 4 & $t$ & $t$ & 40,625 & 17.8 \\
\hline Wisconsin & 12 & 61,075 & 57,225 & 39,338 & 21.1 \\
\hline Yale & 16 & 81,748 & 77,557 & 49,500 & 19.6 \\
\hline York & 5 & 68,432 & 68,955 & 43,275 & 13.2 \\
\hline
\end{tabular}




\section{TABLE 43: BEGINNING PROFESSIONAL SALARIES IN ARL UNIVERSITY LAW LIBRARIES \\ RANK ORDER TABLE, FY 2007-2008*}

\begin{tabular}{|c|c|c|c|c|c|}
\hline Rank & Institution & Salary & Rank & Institution & Salary \\
\hline 1 & New York & $\$ 57,566$ & 39 & Florida & $\$ 42,000$ \\
\hline 2 & Boston University & 55,000 & 39 & Georgia & 42,000 \\
\hline 2 & George Washington & 55,000 & 39 & Minnesota & 42,000 \\
\hline 2 & Hawaii & 55,000 & 39 & Pennsylvania & 42,000 \\
\hline 5 & Cornell & 54,000 & 43 & Saskatchewan & 41,598 \\
\hline 6 & Virginia & 52,700 & 44 & Ohio State & 41,500 \\
\hline 7 & Rutgers, Camden Law & 52,000 & 45 & Manitoba & 41,331 \\
\hline 8 & Columbia & 51,500 & 46 & Pennsylvania State & 41,200 \\
\hline 9 & Illinois, Urbana & 51,000 & 47 & Boston College & 41,050 \\
\hline 10 & Arizona & 50,000 & 48 & Temple & 40,800 \\
\hline 10 & Florida State & 50,000 & 49 & Queen's & 40,695 \\
\hline 10 & Houston & 50,000 & 50 & McGill & 40,625 \\
\hline 10 & Rutgers, Newark Law & 50,000 & 50 & Western Ontario & 40,625 \\
\hline 10 & South Carolina & 50,000 & 52 & Cincinnati & 40,425 \\
\hline 10 & Southern Illinois & 50,000 & 53 & Calif. Davis & 40,008 \\
\hline 10 & Washington & 50,000 & 53 & Calif. Los Angeles & 40,008 \\
\hline 17 & Yale & 49,500 & 55 & Alabama & 40,000 \\
\hline 18 & Washington-St .Louis & 49,000 & 55 & Indiana & 40,000 \\
\hline 19 & New Mexico & 48,000 & 55 & Iowa & 40,000 \\
\hline 20 & Michigan & 47,218 & 55 & Louisiana State & 40,000 \\
\hline 21 & Harvard & 47,100 & 55 & Northwestern & 40,000 \\
\hline 22 & Georgetown & 46,500 & 55 & Notre Dame & 40,000 \\
\hline 23 & British Columbia & 45,784 & 55 & Oregon & 40,000 \\
\hline 24 & Duke & 45,000 & 55 & Tennessee & 40,000 \\
\hline 24 & North Carolina & 45,000 & 63 & Wisconsin & 39,338 \\
\hline 24 & SUNY Buffalo & 45,000 & 64 & Connecticut & 39,263 \\
\hline 24 & Texas Tech & 45,000 & 65 & Oklahoma & 39,000 \\
\hline 24 & Utah & 45,000 & 65 & Vanderbilt & 39,000 \\
\hline 24 & Wayne State & 45,000 & 67 & Kentucky & 38,500 \\
\hline 30 & Montreal & 44,630 & 67 & Missouri & 38,500 \\
\hline 31 & Colorado & 44,000 & 69 & Louisville & 37,000 \\
\hline 31 & Emory & 44,000 & 69 & Texas & 37,000 \\
\hline 31 & Miami & 44,000 & 69 & Tulane & 37,000 \\
\hline 34 & Howard & 43,779 & 72 & Case Western Reserve & 35,000 \\
\hline 35 & York & 43,275 & 72 & Nebraska & 35,000 \\
\hline 36 & Toronto & 43,010 & 74 & Kansas & 33,000 \\
\hline 37 & Arizona State & 43,000 & & \multirow[t]{2}{*}{ Syracuse } & \multirow[t]{2}{*}{ U/A } \\
\hline 38 & Alberta & 42,997 & & & \\
\hline
\end{tabular}

* Beginning salary figures represent officially designated base, not necessarily salaries of actual incumbents.

$\dagger$ Canadian salaries expressed in U.S. dollars. 


\section{TABLE 44: MEDIAN PROFESSIONAL SALARIES \\ IN ARL UNIVERSITY LAW LIBRARIES \\ RANK ORDER TABLE, FY 2007-2008*}

\begin{tabular}{|c|c|c|c|c|c|}
\hline Rank & Institution & Salary & Rank & Institution & Salary \\
\hline 1 & California, Davis & $\$ 92,436$ & 37 & Virginia & $\$ 60,000$ \\
\hline 2 & Toronto & 84,084 & 38 & Texas Tech & 59,474 \\
\hline 3 & California, Los Angeles & 79,968 & 39 & Tennessee & 59,440 \\
\hline 4 & Hawaii & 79,376 & 40 & Vanderbilt & 57,976 \\
\hline 5 & Boston College & 78,408 & 41 & Nebraska & 57,917 \\
\hline 6 & Yale & 77,557 & 42 & Northwestern & 57,684 \\
\hline 7 & New Mexico & 75,000 & 43 & Colorado & 57,597 \\
\hline 8 & George Washington & 73,266 & 44 & Alabama & 57,349 \\
\hline 9 & Rutgers, Camden & 72,991 & 45 & Wisconsin & 57,225 \\
\hline 10 & New York University & 72,546 & 46 & Miami & 55,432 \\
\hline 11 & Duke & 71,900 & 47 & Tulane & 55,310 \\
\hline 12 & Harvard & 71,380 & 48 & Southern Illinois & 55,146 \\
\hline 13 & Michigan & 71,328 & 49 & Missouri & 55,122 \\
\hline 14 & Columbia & 71,016 & 50 & Emory & 54,422 \\
\hline 15 & SUNY Buffalo & 70,228 & 51 & Texas & 54,288 \\
\hline 16 & Connecticut & 69,788 & 52 & Louisville & 53,853 \\
\hline 17 & York & 68,955 & 53 & Louisiana State & 53,698 \\
\hline 18 & Pennsylvania State & 68,760 & 54 & Florida & 53,556 \\
\hline 19 & Cornell & 68,650 & 55 & Syracuse & 53,310 \\
\hline 20 & Illinois, Urbana & 68,313 & 56 & Temple & 53,043 \\
\hline 21 & Washington & 67,956 & 57 & Oregon & 51,951 \\
\hline 22 & Georgetown & 67,950 & 58 & Kentucky & 51,868 \\
\hline 23 & South Carolina & 66,950 & 59 & Florida State & 51,500 \\
\hline 24 & Iowa & 66,500 & 60 & Georgia & 50,887 \\
\hline 25 & North Carolina & 66,010 & 61 & Cincinnati & 50,000 \\
\hline 26 & Minnesota & 65,500 & 62 & Howard & 48,460 \\
\hline 27 & Utah & 65,265 & 63 & Houston & 48,000 \\
\hline 28 & Indiana & 64,176 & 64 & Oklahoma & 47,521 \\
\hline 29 & Ohio State & 63,963 & 65 & Wayne State & 43,450 \\
\hline 30 & Arizona & 63,854 & 66 & Kansas & 43,000 \\
\hline 31 & Case Western Reserve & 62,791 & & & \\
\hline 32 & Notre Dame & 62,200 & & & \\
\hline 33 & Pennsylvania & 61,815 & & & \\
\hline 34 & Rutgers, Newark & 61,279 & & & \\
\hline 35 & Boston University & 61,000 & & & \\
\hline 36 & Arizona State & 60,966 & & & \\
\hline
\end{tabular}

* Salaries of directors are not included in the calculation of medians. Alberta, British Columbia, McGill, Manitoba, Montreal, Queen's, Saskatchewan, Washington U.-St. Louis, and Western Ontario are not ranked because they reported fewer than four individuals.

$\dagger$ Canadian salaries expressed in U.S. dollars. 


\section{TABLE 45: AVERAGE PROFESSIONAL SALARIES IN ARL UNIVERSITY LAW LIBRARIES \\ RANK ORDER TABLE, FY 2007-2008*}

\begin{tabular}{|c|c|c|c|c|c|}
\hline Rank & Institution & Salary & Rank & Institution & Salary \\
\hline 1 & California, Davis & $\$ 89,280$ & 37 & Case Western Reserve & $\$ 62,667$ \\
\hline 2 & Yale & 81,748 & 38 & Illinois, Urbana & 62,582 \\
\hline 3 & George Washington & 81,629 & 39 & Virginia & 62,336 \\
\hline 4 & Toronto & 80,540 & 40 & Southern Illinois & 62,040 \\
\hline 5 & Hawaii & 79,336 & 41 & Louisiana State & 61,972 \\
\hline 6 & California, Los Angeles & 77,232 & 42 & Temple & 61,754 \\
\hline 7 & Michigan & 77,070 & 43 & Texas & 61,677 \\
\hline 8 & Harvard & 75,589 & 44 & Utah & 61,380 \\
\hline 9 & New York University & 75,491 & 45 & Texas Tech & 61,269 \\
\hline 10 & Columbia & 74,710 & 46 & Wisconsin & 61,075 \\
\hline 11 & Georgetown & 73,964 & 47 & Northwestern & 59,235 \\
\hline 12 & Cornell & 73,209 & 48 & Nebraska & 58,323 \\
\hline 13 & Boston College & 73,004 & 49 & Florida & 57,474 \\
\hline 14 & Minnesota & 72,812 & 50 & Cincinnati & 57,367 \\
\hline 15 & Duke & 72,106 & 51 & Louisville & 56,866 \\
\hline 16 & Pennsylvania State & 70,980 & 52 & Syracuse & 56,574 \\
\hline 17 & Iowa & 70,570 & 53 & Miami & 56,240 \\
\hline 18 & New Mexico & 69,741 & 54 & Alabama & 56,059 \\
\hline 19 & Rutgers, Newark & 69,399 & 55 & Tulane & 55,909 \\
\hline 20 & Tennessee & 69,284 & 56 & Emory & 55,126 \\
\hline 21 & SUNY Buffalo & 69,026 & 57 & Georgia & 53,614 \\
\hline 22 & North Carolina & 68,749 & 58 & Oregon & 53,094 \\
\hline 23 & Connecticut & 68,743 & 59 & Florida State & 52,937 \\
\hline 24 & York $\dagger$ & 68,432 & 60 & Oklahoma & 52,831 \\
\hline 25 & Notre Dame & 68,424 & 61 & Kentucky & 52,734 \\
\hline 26 & Rutgers, Camden & 68,312 & 62 & Houston & 52,302 \\
\hline 27 & Washington & 68,048 & 63 & Missouri & 52,200 \\
\hline 28 & Colorado & 68,030 & 64 & Kansas & 47,828 \\
\hline 29 & Pennsylvania & 67,577 & 65 & Howard & 47,556 \\
\hline 30 & Indiana & 67,432 & 66 & Wayne State & 44,943 \\
\hline 31 & Ohio State & 66,768 & & & \\
\hline 32 & Boston University & 64,222 & & & \\
\hline 33 & South Carolina & 64,176 & & & \\
\hline 34 & Arizona & 63,672 & & & \\
\hline 35 & Vanderbilt & 63,567 & & & \\
\hline 36 & Arizona State & 63,087 & & & \\
\hline
\end{tabular}

* Salaries of directors are not included in the calculation of averages. Alberta, British Columbia, McGill, Manitoba, Montreal, Queen's, Saskatchewan, Washington U.-St. Louis, and Western Ontario are not ranked because they reported fewer than four individuals.

† Canadian salaries expressed in U.S. dollars. 


\section{TABLE 46: NUMBER AND AVERAGE SALARIES OF ARL UNIVERSITY LAW LIBRARIANS \\ BY POSITION AND SEX, FY 2007-2008*}

\begin{tabular}{|c|c|c|c|c|c|c|c|}
\hline \multirow{2}{*}{\multicolumn{2}{|c|}{ Position }} & \multicolumn{2}{|c|}{ Women } & \multicolumn{2}{|c|}{ Men } & \multicolumn{2}{|c|}{ Total } \\
\hline & & Salary & No. & Salary & No. & Salary & No. \\
\hline \multicolumn{2}{|l|}{ Head, Law } & $\$ 95,375$ & 32 & $\$ 93,235$ & 24 & $\$ 94,458$ & 56 \\
\hline \multicolumn{2}{|c|}{ Associate Director } & 80,196 & 29 & 75,680 & 7 & 79,318 & 36 \\
\hline \multicolumn{2}{|c|}{ Assistant Director } & 141,438 & 36 & 150,388 & 36 & 145,913 & 72 \\
\hline \multicolumn{2}{|c|}{ Functional Specialist } & 56,513 & 27 & 59,065 & 28 & 57,813 & 55 \\
\hline \multicolumn{2}{|c|}{ Subject Specialist } & 69,426 & 28 & 67,012 & 15 & 68,584 & 43 \\
\hline \multirow[t]{9}{*}{ Dept. Head: } & Acquisitions & 60,401 & 24 & 61,375 & 10 & 60,687 & 34 \\
\hline & Reference & 77,893 & 20 & 72,450 & 10 & 76,079 & 30 \\
\hline & Cataloging & $\dagger$ & 25 & $\dagger$ & 2 & 70,465 & 27 \\
\hline & Serials & $\dagger$ & 8 & $\dagger$ & 3 & 67,311 & 11 \\
\hline & Documents/Maps & $\dagger$ & 9 & $\dagger$ & 1 & 63,357 & 10 \\
\hline & Circulation & 61,933 & 22 & 56,878 & 6 & 60,850 & 28 \\
\hline & Rare Books/Manuscripts & $\dagger$ & 2 & $\dagger$ & 2 & 93,739 & 4 \\
\hline & Computer Systems & 71,118 & 5 & 75,557 & 5 & 73,338 & 10 \\
\hline & Other & 71,959 & 18 & 71,547 & 11 & 71,803 & 29 \\
\hline \multirow[t]{4}{*}{ Reference: } & Over 14 years experience & 69,563 & 41 & 66,689 & 27 & 68,422 & 68 \\
\hline & 10 to 14 years experience & 62,023 & 19 & 57,903 & 8 & 60,802 & 27 \\
\hline & 5 to 9 years experience & 54,780 & 27 & 59,758 & 13 & 56,398 & 40 \\
\hline & Under 5 years experience & 55,890 & 49 & 55,332 & 24 & 55,707 & 73 \\
\hline \multirow[t]{4}{*}{ Cataloging: } & Over 14 years experience & 63,153 & 23 & 65,569 & 7 & 63,717 & 30 \\
\hline & 10 to 14 years experience & $\dagger$ & 6 & $\dagger$ & 1 & 50,184 & 7 \\
\hline & 5 to 9 years experience & $\dagger$ & 6 & $\dagger$ & 2 & 51,127 & 8 \\
\hline & Under 5 years experience & $\dagger$ & 8 & $\dagger$ & 1 & 50,700 & 9 \\
\hline \multirow[t]{4}{*}{ Other: } & Over 14 years experience & 57,199 & 8 & 62,396 & 4 & 58,931 & 12 \\
\hline & 10 to 14 years experience & $\dagger$ & 3 & N/A & N/A & 60,065 & 3 \\
\hline & 5 to 9 years experience & $\dagger$ & 4 & $\dagger$ & 1 & 53,303 & 5 \\
\hline & Under 5 years experience & $\dagger$ & 4 & $\dagger$ & 1 & 45,856 & 5 \\
\hline \multicolumn{2}{|c|}{ All Positions } & $\$ 72,074$ & 483 & $\$ 78,767$ & 249 & $\$ 74,350$ & 732 \\
\hline
\end{tabular}

* Canadian salaries expressed in U.S. dollars.

N/A - No positions were reported in this category.

$\uparrow$ Salary data are not published when fewer than four individuals are involved in either category. 


\section{TABLE 47: NUMBER AND AVERAGE YEARS OF EXPERIENCE OF ARL UNIVERSITY LAW LIBRARIANS \\ BY POSITION AND SEX, FY 2007-2008}

\begin{tabular}{|c|c|c|c|c|c|c|c|}
\hline \multirow{2}{*}{\multicolumn{2}{|c|}{ Position }} & \multicolumn{2}{|c|}{ Women } & \multicolumn{2}{|c|}{ Men } & \multicolumn{2}{|c|}{ Total } \\
\hline & & Years & No. & Years & No. & Years & No. \\
\hline \multicolumn{2}{|l|}{ Head, Law } & 25.0 & 32 & 17.6 & 24 & 21.8 & 56 \\
\hline \multicolumn{2}{|c|}{ Associate Director } & 21.5 & 29 & 21.1 & 7 & 21.4 & 36 \\
\hline \multicolumn{2}{|c|}{ Assistant Director } & 27.1 & 36 & 25.0 & 36 & 26.0 & 72 \\
\hline \multicolumn{2}{|c|}{ Functional Specialist } & 14.1 & 27 & 9.3 & 28 & 11.7 & 55 \\
\hline \multicolumn{2}{|c|}{ Subject Specialist } & 20.9 & 28 & 15.7 & 15 & 19.0 & 43 \\
\hline \multirow[t]{9}{*}{ Dept. Head: } & Acquisitions & 17.8 & 24 & 15.3 & 10 & 17.0 & 34 \\
\hline & Reference & 15.9 & 20 & 18.5 & 10 & 16.8 & 30 \\
\hline & Cataloging & 25.1 & 25 & 12.5 & 2 & 24.2 & 27 \\
\hline & Serials & 16.8 & 8 & 11.3 & 3 & 15.3 & 11 \\
\hline & Documents/Maps & 26.9 & 9 & 10.0 & 1 & 25.2 & 10 \\
\hline & Circulation & 16.2 & 22 & 10.2 & 6 & 14.9 & 28 \\
\hline & Rare Books/Manuscripts & 20.0 & 2 & 14.0 & 2 & 17.0 & 4 \\
\hline & Computer Systems & 20.8 & 5 & 20.6 & 5 & 20.7 & 10 \\
\hline & Other & 20.2 & 18 & 17.0 & 11 & 19.0 & 29 \\
\hline \multicolumn{2}{|c|}{ Public Services } & 19.0 & 3 & 10.7 & 3 & 14.8 & 6 \\
\hline \multicolumn{2}{|c|}{ Technical Services } & 13.0 & 9 & 24.0 & 1 & 14.1 & 10 \\
\hline \multicolumn{2}{|c|}{ Administrative Services } & 14.6 & 7 & 19.0 & 2 & 15.6 & 9 \\
\hline \multicolumn{2}{|c|}{ Reference } & 11.7 & 136 & 11.8 & 72 & 11.7 & 208 \\
\hline \multicolumn{2}{|l|}{ Cataloger } & 17.6 & 43 & 19.5 & 11 & 18.0 & 54 \\
\hline \multicolumn{2}{|c|}{ All Positions } & 17.8 & 483 & 15.7 & 249 & 17.1 & 732 \\
\hline
\end{tabular}




\section{TABLE 48: NUMBER AND AVERAGE SALARIES OF \\ ARL UNIVERSITY LAW LIBRARIANS \\ BY YEARS OF EXPERIENCE AND SEX, FY 2007-2008*}

\begin{tabular}{lccccccc}
\hline & \multicolumn{2}{c}{ Women } & \multicolumn{2}{c}{ Men } & \multicolumn{2}{c}{ Total } & \multicolumn{2}{c}{ of } \\
Experience & Salary & No. & Salary & No. & Salary & No. & Total \\
\hline $0-3$ years & $\$ 53,825$ & 71 & $\$ 52,956$ & 39 & $\$ 53,517$ & 110 & $15 \%$ \\
$4-7$ years & 59,333 & 54 & 62,713 & 31 & 60,566 & 85 & $12 \%$ \\
$8-11$ years & 63,742 & 51 & 67,210 & 33 & 65,104 & 84 & $11 \%$ \\
$12-15$ years & 65,617 & 55 & 81,094 & 21 & 69,894 & 76 & $10 \%$ \\
$16-19$ years & 82,607 & 39 & 79,148 & 31 & 81,075 & 70 & $10 \%$ \\
$20-23$ years & 73,996 & 34 & 77,104 & 31 & 75,478 & 65 & $9 \%$ \\
$24-27$ years & 79,165 & 50 & 105,345 & 23 & 87,414 & 73 & $10 \%$ \\
$28-31$ years & 83,534 & 54 & 106,948 & 22 & 90,312 & 76 & $10 \%$ \\
$32-35$ years & 90,474 & 50 & 96,034 & 15 & 91,757 & 65 & $9 \%$ \\
over 35 years & $\dagger$ & 25 & $\dagger$ & 3 & 100,655 & 28 & $4 \%$ \\
\hline All Positions & $\mathbf{\$ 7 2 , 0 7 4}$ & $\mathbf{4 8 3}$ & $\mathbf{\$ 7 8 , 7 6 7}$ & $\mathbf{2 4 9}$ & $\mathbf{\$ 7 4 , 3 5 0}$ & $\mathbf{7 3 2}$ & $\mathbf{1 0 0 \%}$ \\
\hline
\end{tabular}

* Canadian salaries expressed in U.S. dollars. 



\section{UNIVERSITY LIBRARY}

Questionnaire and Instructions 


\section{ARL Annual Salary Survey 2007-2008 \\ University Library Questionnaire \\ General And Data Input (Excel) Instructions}

http://www.arl.org/stats/annualsurveys/salary/

\section{General Overview}

- Use the newly available Web form for your data submission. Fill in Part I on the Web and upload your file for Part II through the same interface.

NOTE: You must complete the entire submission in a single session. The Web interface does NOT allow you to return and edit your information once it is submitted.

- This survey is concerned with professional positions only. Since the criteria for determining professional status vary among libraries, there is no attempt to define the term "professional." Each library should report the salaries of those staff members it considers professionals, irrespective of faculty status or membership in a collective bargaining unit, including, when appropriate, staff who are not librarians in the strict sense of the term, such as computer experts, systems analysts, budget officers, etc.

- Report individual salaries for the Main, Law, and Medical library on the separate template using Microsoft Excel (see http://www.arl.org/stats/annualsurveys/salary/salform07.shtml). A generic template is available. Add your institution's ARL Library Institution Code [LibID]. (See http://www.arl.org/stats/annualsurveys/surveycoord/instno_inam.shtml if you do not know your code.)

- Use "Percent" to determine if an employee works full-time or part-time. All full-time employees have Percent $=1.00$, i.e., they work $100 \%$ of a full-time schedule. If Percent is less than 1.00, then the employee works that fraction of a full-time schedule. For example, a $65 \%$ time appointment would be entered as 0.65 . Calculate the percent appointment by dividing the amount of time an employee works by the amount considered to be the norm for full-time employment at your institution. For example, if a full-time appointment at your institution is 12 months at 40 hours per week:

○ A 9-month part-time appointment has Percent $=9 / 12$, or 0.75 .

○ An appointment at 30 hours per week has Percent $=30 / 40$, also 0.75.

$\circ \quad$ An appointment at 30 hours and 9 months has Percent $=0.75 \times 0.75=0.56$.

Enter Percent with two decimal points.

- Report salaries for both full-time and part-time professional positions. Salaries for part-time positions should NOT be converted to their full-time equivalents. Report the actual part-time salary paid and indicate the percent appointment for that employee in the appropriate column.

- Include salaries for all professional positions, regardless of whether the salaries come from regular library budget funds or from special funds such as research grants. Please include all professionals involved in the provision of library services, including contract-supported positions.

- The salary figures should be straight gross salary figures. Do not include fringe benefits. 
- Provide explanatory footnotes to the reported figures, when necessary, at the end of Part I. Footnotes will be included in the published survey, where appropriate.

- After all data have been entered, make a backup copy of the complete file for your institution's master file. Your backup should include individual names/ID numbers. NOTE: The data submitted to ARL should NOT include individual names/ID numbers, so ARL will NOT be able to supply a copy of your institution's complete file next year.

- Please return the questionnaire the ARL Statistics and Measurement Program by September 30, 2007. Be sure to keep a complete copy of your return, including the electronic version of the data for your files.

\section{INSTRUCTIONS}

\section{Part I: Summary Data}

1. Part I of this survey deals with general information for the current fiscal year, 2007-08.

2. Include the Beginning Professional Salary for Law and Medical libraries if included in the survey.

3. The Beginning Professional Salary is the salary that would be paid to a newly hired professional without experience, not necessarily the lowest professional salary paid. In reporting the beginning salary, please use a figure that is actually used or likely to be used for entry-level librarians hired by your library, even if it is your practice rarely to hire entry-level professionals without experience.

4. Please report the 2007-2008 Beginning Professional Salary to the best of your knowledge as it exists on July 1, 2007. Do not delay returning your survey with the expectation that more information will be available later.

5. The 2007-2008 Average and Median Salary figures will be calculated by ARL from the individual data supplied.

6. Be sure to fill in the name of the reporting library and the name of the person who prepares the report.

\section{Part II: Individual Data}

1. Part II of this survey requests information on salary, sex, minority status, rank, and years of experience for all filled positions for fiscal year 2007-2008. The survey requests information for individuals; aggregate data for each institution will be generated by computer. Vacant positions should be excluded from your report.

2. Data for the Main, Law, and Medical libraries should be reported on separate Excel files.

3. Obtain the Excel file. These instructions assume that you have Microsoft Excel available for use. If not, or if you have trouble opening the files in Excel, please call the ARL Statistics and Measurement Program at (202) 296-2296 or email stats-ra@arl.org.

4. The template Excel file is available at: http://www.arl.org/stats/annualsurveys/salary/salform07. shtml. This is a generic, blank file that can hold data for Main, Law, or Medical libraries. The file's name is "sal07xxxx.xls"; open the file and save it to your own computer by choosing "Save 
As" under the File menu. When saving the file, please change the "xxxx" in its name to your institution's name, e.g., "sal07ALABAMA.xls."

The file contains columns labeled as follows:

Required: Name/ID\# LibID Page Line Salary Job Sex OEOcat Yrsexp Rank Percent Optional: Hisp NatAm Asian Black HawPI White

In the LibID column, enter your ARL Library Institution Code. (See http://www.arl.org/stats/ annualsurveys/surveycoord/instno_inam.shtml if you do not know your code.) If you leave this column blank we will fill it in for you when we receive the data.

Columns labeled "Page," "Line," and "Percent" are already filled for you. The numbers in the "Page" and "Line" columns will be used to identify these positions in case of data errors; do not change them. Ten "pages" of 25 lines each have been provided; if this is not sufficient to list all positions at your institution, copy and paste lines 1-25 of the last page as needed.

\section{Entering Data for Part II: Individual Data}

1. The "Name/ID\#" column is for your internal use, to enter and verify information for staff members by name. ARL does not require that you submit the information in this field to ARL. Please delete this column before sending the file to ARL. Upon receiving this file, ARL will delete any data in this column if you have not deleted them already.

2. The "LibID" will hold your institution's ARL number, for identification purposes. If you do not know your ARL number, you can find it on the Web under ARL Library Institution Codes. If you leave this column blank, it will be filled in by ARL staff.

3. "Salary" should be entered as it existed on July 1, 2007. Please do not hold up the reporting process for later salary adjustments. Include all filled positions and exclude all vacant positions. Report the actual salary paid. Do not adjust part-time salaries to their full-time equivalents; ARL will do this during the data analysis and verification stage. Do not include fringe benefits.

4. Each position can have only one "Job" code, to be taken from the following list:

DIRLIB Director of Libraries (includes Dean of Libraries and equivalent titles)

ASCDIR Associate Director

ASTDIR Assistant Director

HDMED Head, Medical Library (Human Medicine only)

HDLAW Head, Law Library

HDBR Head, Other Branch Library (including Veterinary Medicine)

FSPEC Functional Specialist
$\mathrm{ARCH}$
Archivists/Curators
BUSI
Budget/Fiscal/Business Manager/Facilities
HUMRES
Human Resources/Training/Staff Development
ITS
Information Technology Systems
ITW
Information Technology Web Development 


\section{ITP Information Technology Programming/Application Development \\ MEDIA Media/Multimedia Specialists (including graphics) \\ PRES Preservation/Conservation}

$\begin{array}{ll}\text { SSPEC } & \text { Subject Specialist } \\ \text { HDACQ } & \text { Head, Acquisitions Department } \\ \text { HDCAT } & \text { Head, Catalog Department/Unit } \\ \text { HDCIRC } & \text { Head, Circulation } \\ \text { HDCOMP } & \text { Head, Library and Computer Systems } \\ \text { HDDOC } & \text { Head, Documents Department } \\ \text { HDMAP } & \text { Head, Map Room/Department } \\ \text { HDRBM } & \text { Head, Rare Book/Manuscripts Department } \\ \text { HDREF } & \text { Head, Reference Department } \\ \text { HDSER } & \text { Head, Serials Department } \\ \text { HDOTH } & \text { Head, Other Department/Service/Agency } \\ \text { CAT } & \text { Catalogers, both general and specialized } \\ \text { REF } & \text { Reference librarians, both general and specialized } \\ \text { PUBS } & \text { Public Services, non-supervisory, except reference librarians } \\ \text { TECH } & \text { Technical Services, non-supervisory, except catalogers } \\ \text { ADMIN } & \text { Administrative and other units, non-supervisory position }\end{array}$

The position categories used in this survey are intended to correspond roughly with the activities carried on in libraries, not with any particular pattern of staff organization or nomenclature. Please use these categories in the manner you feel best applies to your library. If any individual has responsibilities described by more than one of the above categories, choose the category that is most typical of his/her general duties.

Associate or Assistant Director, and Head, Other Branch. Use these codes for all persons at these levels regardless of the area of specialty. If an assistant or associate director is also head of a department, choose the category that most reflects the general duties of the person currently in the position.

Specialists. These are of two kinds: Subject Specialists primarily build collections, but may also offer specialized reference and bibliographic services; Functional Specialists are media specialists or experts in management fields such as personnel, fiscal matters, systems, preservation, etc. Specialists may not be, strictly speaking, professional librarians (i.e., have an MLS). The "specialist" category would generally not be used for someone with significant supervisory responsibilities, who should instead be listed as a department head or assistant director (see also note under Assistant Department Head, below).

Functional Specialist sub-codes. Starting with the 2004-2005 Salary Survey, the ARL Statistics and Measurement Committee adopted a proposal from the ACRL Personnel Administrators and Staff Development Officers Discussion Group to break down the Functional Specialist category. For each position which would have been labeled FSPEC prior to 2004-2005, instead please use one of the eight sub-codes (ARCH, BUSI, HUMRES, ITS, ITW, ITP, MEDIA, PRES) to describe that position. If you cannot determine which sub-code to use, please use the FSPEC code. 
Department Heads. Department Heads not specifically included in the above list should be included under the category "Head, Other Department/Service/Agency." Head, Catalog Department should be used either for the department that handles all cataloging, or for the head of a specialized cataloging unit (e.g. copy cataloging or foreign languages). List the head of library automation and computer systems, applications, programming, etc. as HDCOMP unless that person is also an Associate or Assistant Director, in which case use the appropriate administrative code. If there is an intermediate level of management between an Associate or Assistant Director and the professionals who actually carry out the analysis, programming, etc., use HDCOMP to define that intermediate level. Professionals who carry out analysis, programming, etc., should be listed as functional specialists (FSPEC).

Head, Acquisitions Department. Use HDACQ for all of the following positions: (a) head of a department that is responsible for the selection of material (or management of selection activities carried out on a basis encompassing more than a single organizational unit), but not responsible for the placement of orders, payment of invoices, etc.; (b) head of a department responsible for the placement of orders, maintaining on-order files, payment of invoices, etc., but not responsible for selection decisions; (c) head of a department responsible for both the selection decisions (or coordination of selection activities) and for acquiring the material. Libraries that split these two functions between two departments should report more than one professional with the position HDACQ.

[Special note concerning Assistant Department Heads. Assistant Department Heads who are responsible for major units and spend the bulk of their time in supervision and revision of the work of others should also be listed as "Head, Other Department/Service/Agency." See additional subcodes below for Head, Cataloging, and Head, Other Department. However, Assistant Head positions responsible for small units or for supervision only in the absence of the head should be reported as non-supervisory or specialist positions as appropriate.]

Administrative. Please note that ADMIN is not only for Administrative Services and related positions, but also can be applied to Public Relations/Communications, Development/Fundraising, and all other administrative and/or professional positions which do not have a logical home elsewhere.

5. Please indicate "Sex" with the letter M or F, indicating male or female, respectively.

6. "OEOCat" minority status code, for U.S. university libraries only, should be indicated with one of the following code numbers. (Leave blank if a Canadian library):

$$
\begin{aligned}
& 1=\text { Black } \\
& 2=\text { Hispanic } \\
& 3=\text { Asian or Pacific Islander } \\
& 4=\text { American Indian or Native Alaskan } \\
& 5=\text { Caucasian/Other }
\end{aligned}
$$

7. "YrsExp," or total years of professional experience. For most professional staff members this will mean counting the years since the MLS degree was awarded. When counting, do not subtract 
interim periods when an individual was not engaged in professional library employment if these periods are short in relation to the overall professional career. Count an academic year contract period as a full year. Be sure to include professional experience in previous positions and in other institutions. The figure should be rounded off to the nearest whole number; for example, a position with 14.5 years of experience would appear as 15 .

\section{Indicate "Rank" using the following system of codes:}

0 The library director. Some systems also use 0 for assistant and/or associate directors.

1 Lowest level in the rank structure, such as an entry-level position.

2-8 Successively higher levels; for example, 5 indicates a higher rank than 2.

9 Rank cannot be determined, or, the individual is outside the organization's rank structure.

Responses concerning rank should be limited to professional librarians, and other professionals who occupy the same ranks as librarians. Leave the rank column blank for professionals who do not occupy these ranks or if the column is not applicable. For example, if the Library Business Officer holds a rank typically used for university administrators but not for librarians, do not supply a rank code for that individual, even if you have included salary and other data.

If multiple ranking structures are used for librarians and these structures are substantially different and not equivalent, enter individual rank information only for that group which represents the largest fraction of "rank-and-file" librarians.

The maximum number of ranks reported here should not exceed the maximum number of ranklevels reported in Part I for individual data under Rank structure. When counting the total number of rank levels, include ranks that may be unoccupied at the present time due to circumstances like unusually high turnover, hiring freezes, etc.

9. "Percent" is used to determine if an employee works full-time or part-time. All full-time employees have Percent $=1.00$, i.e., they work $100 \%$ of a full-time schedule. If Percent is less than 1.00, then the employee works that fraction of a full-time schedule. For example, a $65 \%$ time appointment would be entered as 0.65 . Calculate the percent appointment by dividing the amount of time an employee works by the amount considered to be the norm for full-time employment at your institution. For example, if a full-time appointment at your institution is 12 months at 40 hours per week:

○ A 9-month part-time appointment has Percent $=9 / 12$, or 0.75 .

$\circ$ An appointment at 30 hours per week has Percent $=30 / 40$, also 0.75 .

$\circ$ An appointment at 30 hours and 9 months has Percent $=0.75 \times 0.75=0.56$.

Enter Percent with two decimal points.

Optional Questions: The U.S. Office of Management and Budget has revised the Standards for the Classification of Federal Data on Race and Ethnicity ${ }^{1}$ and according to the new standard there will be five minimum categories for data on race (American Indian or Alaska Native, Asian, Black or African American, Native Hawaiian or Other Pacific Islander, and White) and one category for data on ethnicity ("Hispanic or Latino"). Respondents will be able to report more than one race

\footnotetext{
${ }^{1}$ http:/ / www.whitehouse.gov/omb/bulletins/b00-02.html and http:/ / www.whitehouse.gov/OMB/fedreg/ombdir15.html
} 
by choosing multiple responses to the race question. The purpose of the revised classification is to reflect the increasing diversity of the U.S. population that has resulted primarily from growth in immigration and in interracial marriages. The new standards were used by the Bureau of the Census in the 2000 decennial census. In light of these developments, we are collecting the new classification on race and ethnicity in the ARL Annual Salary Survey on an optional basis.

Ethnicity should be indicated by coding 1 to indicate if the person is of Hispanic or Latino ethnicity, and coding 0 otherwise. The definition of Hispanic or Latino ethnicity is: A person of Cuban, Mexican, Puerto Rican, Cuban, South or Central American, or other Spanish culture or origin, regardless of race.

Race should be indicated for U.S. university libraries only, by choosing one or more responses among the five racial categories provided here; $1=$ yes and $0=$ no. You can select multiple racial categories for a person. The definitions of the five racial categories, listed with their respective column names, are:

American Indian or Alaska Native (NatAm): A person having origins in any of the original peoples of North and South America (including Central America) who maintains tribal affiliation or community attachment.

Asian (Asian): A person having origins in any of the original peoples of the Far East, Southeast Asia, or the Indian subcontinent including, for example, Cambodia, China, India, Japan, Korea, Malaysia, Pakistan, the Philippine Islands, Thailand, and Vietnam.

Black or African American (Black): A person having origins in any of the black racial groups of Africa.

Native Hawaiian or Other Pacific Islander (HawPI): A person having origins in any of the original peoples of Hawaii, Guam, Samoa, or other Pacific Islands.

White (White): A person having origins in any of the original peoples of Europe, the Middle East, or North Africa.

\section{Submitting the Data for Part I and Part II on the Web}

ARL is using the online services of FormSpring to collect the data. As part of its privacy policy, FormSpring pledges not to sell any collected information to third parties. For the complete FormSpring privacy policy, visit http://www.formspring.com/privacy.html. ARL also accepts Part I and Part II of the salary survey by e-mail from those users who may be uncomfortable submitting the files in FormSpring.

Access the form at: http://www.arl.org/stats/annualsurveys/salary/salform07.shtml. Be sure to have the electronic copy of your completed salary survey Excel file handy as you will be submitting this file via the FormSpring Web form. In addition to the completed Excel file, be prepared to provide the following information as well: 
- The name, title, email and phone number of the person who prepared the Excel file. The name, title, email and phone number of your institution's contact person for the salary survey (if different from the person who prepared the Excel file)

- Indicate whether you are submitting salary information for one or more of the following: Main, Law, or Medical library, and the beginning professional salary and rank structure for each.

For professional salary list the salary that would be paid to a newly hired professional without experience (even if local practice discourages hiring entry-level professionals without experience). Please report the 2007-2008 beginning professional salary to the best of your knowledge as it existed on July 1, 2007.

For rank structure, list the number of unique levels in your institution's rank structure. If you have no levels in your rank structure, use 1. The number reported here should be equal to the highest number in the "Rank" column of your Excel file (i.e., the number of levels reported in your Excel file should equal the number of levels reported here).

- The names of the libraries that are included and excluded in your figures for the 'general libraries' (these can be main campus libraries or branch campus libraries), as well as any other explanatory information, should be indicated in a footnote. In your footnotes, report any information that would clarify the figures submitted: the inclusion and exclusion of branch campus libraries, a reporting date that is sooner/later than July 1, 2007, etc. Please make an effort to word your footnotes in a manner consistent with notes appearing in the published report, so that ARL can interpret your footnotes correctly.

Please return the completed questionnaire to the ARL Statistics and Measurement Program by September 30, 2007.

For assistance, contact Martha Kyrillidou (martha@arl.org) or Mark Young (stats-ra@arl.org) or Kristina Justh (kristina@arl.org) or Gary Roebuck (gary@arl.org).

Tel. 202-296-2296 or fax 202-872-0884.

\section{http://www.arl.org/stats/annualsurveys/salary/}




\section{ARL Annual Salary Survey 2007-08 \\ UNIVERSITY LIBRARY QUESTIONNAIRE}

Part I: Summary Data

Reporting Institution

Date Returned to ARL

Report Prepared by (name)

Title

Email address

Phone number

Contact person (if different)

Title

E-mail address

Phone number

1. Beginning Professional Salary

Main

Law

Medical

Beginning professional salary for 2006-07

(Note: ARL will calculate the 2007-08 median and average professional salaries for your library from the individual data you supply in Part II of this questionnaire.)

\section{Rank Structure.}

Indicate the number of levels in your institution's rank structure for professional librarians. You should report here the maximum number of rank levels, reported in Part II for individual data, under the Rank column.

1 level (i.e., no differentiated levels)

2 levels

3 levels

4 levels

5 levels

more than 5 levels (please specify the number of levels: 


\section{FOOTNOTES}

3a. Please list which libraries are included in the data submitted for the "general" libraries. These can be main campus libraries or branch campus libraries.

3b. Please list which libraries are NOT included in the data submitted for the "general" libraries. These can be main campus libraries or branch campus libraries.

Please indicate any other explanatory information in footnotes. These additional footnotes, if necessary, should be placed in the space below or on attached pages.

Please return the completed questionnaire to the

ARL Statistics and Measurement Program by September 30, 2007.

For assistance, contact Martha Kyrillidou (martha@arl.org) or Mark Young (stats-ra@arl.org), or call 202-296-2296. 

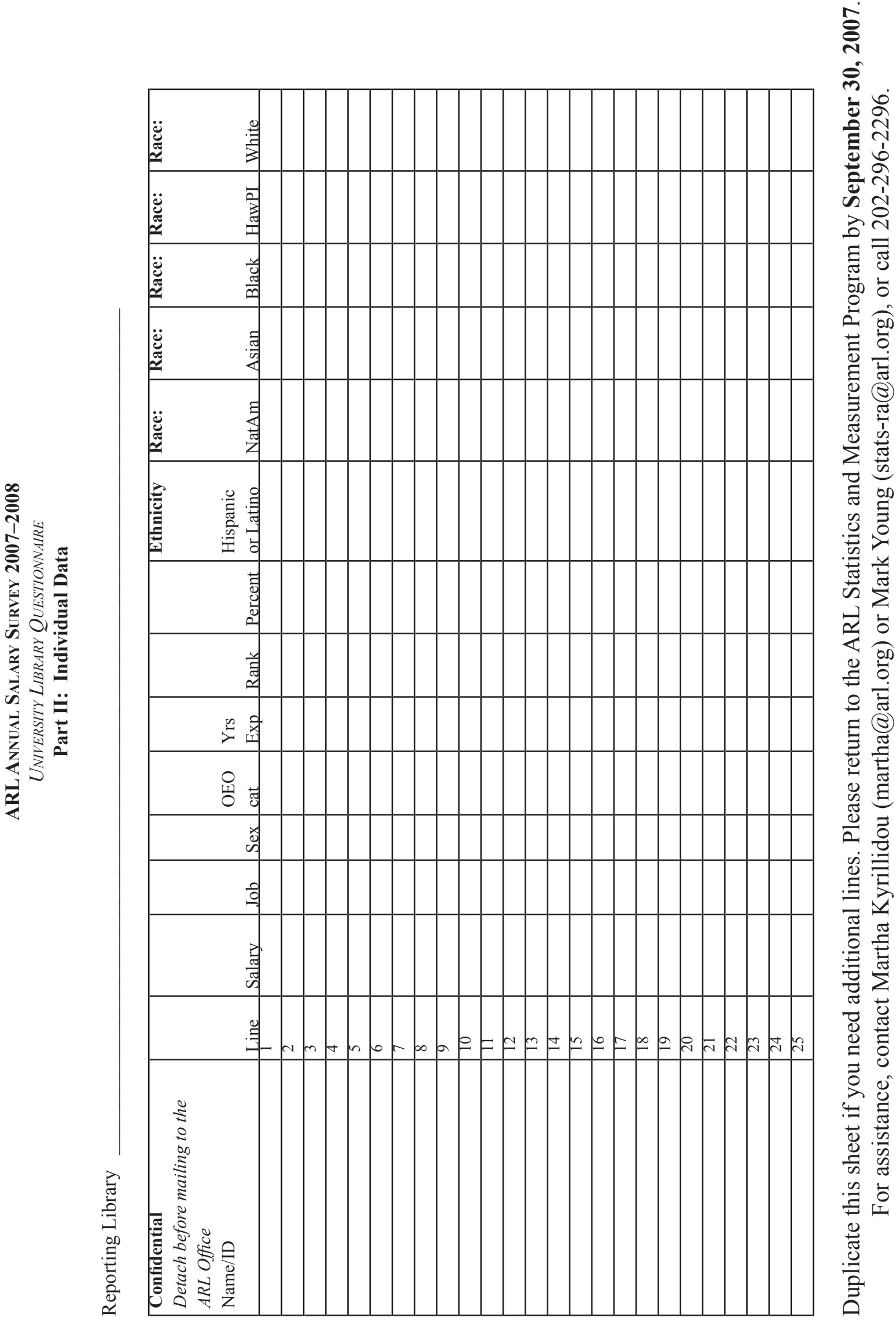
NONUNIVERSITY LIBRARY

Questionnaire and Instructions 


\section{ARL Annual Salary Survey 2007-2008 \\ NONUNIVERSITY LIBRARY QUESTIONNAIRE \\ General Instructions for Completing the Questionnaire}

1. This survey is concerned with the salaries of professional positions only. Since the criteria for determining professional status vary among libraries, there is no attempt to define the term "professional." Each library should report the salaries of those staff members it considers professionals, irrespective of membership in a collective bargaining unit, and including, when appropriate, staff who are not librarians in the strict sense of the term, such as systems analysts, budget officers, etc.

2. Salaries should be reported for all filled positions. Vacant positions should be excluded from your report.

3. Report 2007-08 salaries as they exist on July 1, 2007. If the library normally increases salaries at a date after July 1 , and the salary as of that later date is known or can be estimated (within $\$ 100$ or so) by the time the questionnaire is due to be returned, please use the higher salary and footnote the effective date and/or whether the reported figures are known or estimated. Please do not hold up the reporting process for later salary adjustments.

4. The Median Salary is the salary that has an equal number of salaries above it and below it. In those libraries with an even number of positions, the median salary is the average of the two salaries that have an equal number of salaries above and below them.

5. The Beginning Professional Salary is the salary that would be paid to a professional without experience, not necessarily the lowest professional salary paid. In reporting the beginning salary, please use a figure that is actually used or likely to be used for entry-level librarians hired by your library.

6. Salaries should be reported for both full-time and part-time professional positions. However, salaries for part-time positions should be converted to their full-time equivalents before reporting; do not report the actual part-time salary paid.

7. Salaries should normally be reported on a 12-month basis. If an appointment is for 9 or 10 months at the option of the employee, the actual salary paid should be increased to its 12-month equivalent. However, if appointments of less than 12 months are required by the employer, report the actual salary paid.

8. The salaries for all professional positions should be included, regardless of whether the salaries come from regular library budget funds or from special funds such as research grants.

9. The salary figures should be straight gross salary figures. Do not include fringe benefits.

10. Explanatory footnotes to the reported figures may be provided when necessary. Footnotes will be included in the published survey.

11. Provide the name of the reporting library and the name of the person who prepares the report.

12. Please return the questionnaire to the ARL Statistics and Measurement Program Office by September 30, 2007. 


\section{ARL Annual Salary Survey 2007-2008 \\ NONUNIVERSITY LIBRARY QUESTIONNAIRE}

Part I: Summary Data

Reporting Institution

Date Returned to ARL

Report Prepared by (name)

Title

Email address

Phone number

Contact person (if different)

Title

Email address

Phone number

1. Complete the table on the back of this sheet by indicating the number of filled or temporarily vacant professional positions in each salary range for fiscal years 2006-2007 and 2007-2008.

2. Median professional salary for fiscal year 2007-2008:

3. Beginning professional salary for 2007-2008:

4. Footnotes (please compare with footnotes from surveys of previous years)

a. Law Library salaries are included.

Yes

No

We do not have a Law Library.

b. Medical Library salaries are included.

Yes

No

We do not have a Medical Library.

c. Branch libraries not included (please attach an additional sheet if necessary):

5. Other comments (please attach an additional sheet if necessary): 
Indicate the number of filled professional positions

in each salary range for fiscal years 2006-2007 and 2007-2008.

\begin{tabular}{|c|c|}
\hline \multirow{3}{*}{$\begin{array}{l}\text { Salary Range } \\
\text { More than } 250,000\end{array}$} & Number of Positions \\
\hline & 2006-2007 \\
\hline & \\
\hline \multicolumn{2}{|l|}{$200,000-250,000$} \\
\hline \multicolumn{2}{|l|}{$175,000-199,999$} \\
\hline \multicolumn{2}{|l|}{$150,000-174,999$} \\
\hline \multicolumn{2}{|l|}{$140,000-149,999$} \\
\hline \multicolumn{2}{|l|}{$130,000-139,999$} \\
\hline \multicolumn{2}{|l|}{$120,000-129,999$} \\
\hline \multicolumn{2}{|l|}{$110,000-119,999$} \\
\hline \multicolumn{2}{|l|}{$100,000-109,999$} \\
\hline \multicolumn{2}{|l|}{$95,000-99,999$} \\
\hline \multicolumn{2}{|l|}{$90,000-94,999$} \\
\hline \multicolumn{2}{|l|}{$85,000-89,999$} \\
\hline \multicolumn{2}{|l|}{$80,000-84,999$} \\
\hline \multicolumn{2}{|l|}{$76,000-79,999$} \\
\hline \multicolumn{2}{|l|}{$74,000-75,999$} \\
\hline \multicolumn{2}{|l|}{$72,000-73,999$} \\
\hline \multicolumn{2}{|l|}{$70,000-71,999$} \\
\hline \multicolumn{2}{|l|}{$68,000-69,999$} \\
\hline \multicolumn{2}{|l|}{$66,000-67,999$} \\
\hline \multicolumn{2}{|l|}{$64,000-65,999$} \\
\hline \multicolumn{2}{|l|}{$62,000-63,999$} \\
\hline \multicolumn{2}{|l|}{$60,000-61,999$} \\
\hline \multicolumn{2}{|l|}{$58,000-59,999$} \\
\hline \multicolumn{2}{|l|}{$56,000-57,999$} \\
\hline \multicolumn{2}{|l|}{$54,000-55,999$} \\
\hline \multicolumn{2}{|l|}{$52,000-53,999$} \\
\hline \multicolumn{2}{|l|}{$50,000-51,999$} \\
\hline $48,000-49,999$ & \\
\hline $46,000-47,999$ & \\
\hline $44,000-45,999$ & \\
\hline $42,000-43,999$ & \\
\hline $40,000-41,999$ & \\
\hline $38,000-39,999$ & \\
\hline $36,000-37,999$ & \\
\hline $34,000-35,999$ & \\
\hline $32,000-33,999$ & \\
\hline $30,000-31,999$ & \\
\hline less than 30,000 & \\
\hline
\end{tabular}

Please return the completed questionnaire to the ARL Statistics and Measurement Program by September 30, 2007. 


\section{Footnotes to the ARL Annual Salary Survey, 2007-2008}

INSTITUTION

ALABAMA

ALBERTA

ARIZONA

ARIZONA STATE

AUBURN

BOSTON COLLEGE

BRIGHAM YOUNG

BRITISH COLUMBIA

\section{BROWN}

CALIFORNIA, BERKELEY
Note

Includes Angelo Bruno (Business), McClure (Education), Amelia Gayle

Gorgas (main), W.S. Hoole (Special Collections), and the Eric and Sarah

Rodger (Science and Engineering) libraries.

Includes Bibliographic Services, H.T. Coutts Education Library, Humanities and Social Services Library, Faculty Saint-Jean Library, Winspear Business

Reference Room, the Office of Staff Development and Training, and Cameron Library (which includes Interlibrary Loans/Document Delivery, Financial Systems and Analysis, Science and Technology Library, and Information Technology Services).

Excludes John W. Scott Helath Sciences Library, J.A. Weir Memorial Law Library.

Includes Main Library, Science-Engineering Library, Music Library, and the Center for Creative Photography, Arizona Health Sciences Library in Tucson Arizona Health Sciences Library in Phoenix (branch).

Includes the Tempe (Main) campus, the ASU West campus, and the ASU Polytechnic (East) campus.

Includes all Auburn University Libraries (including the two branch libraries on the main campus).

Includes O'Neill Library (main library), Bapst Art Library, Burns Library of Rare Books, School of Social Work Library, and the Educational Resource Center.

Excludes BYU-Idaho, BYU-Hawaii, BYU Law Library, the LDS Business College, and the Salt Lake Center.

Includes Asian, David Lam (Management), Education, Fine Arts, Koerner, Law, Life Sciences (including Biomedical Branch, Hamber, St. Paul's Hospital, and Woodward Biomedical), MacMillan, Irving K. Barber Learning Centre, Mathematics, Music, Robson Square, and UBC Okanagan libraries, as well as the First Nations House of Learning (Xwi7xwa), the Library Processing Center, the Rare Books and Special Collections Division, and the Science and Engineering Division.

Excludes Reading Rooms and Affiliated Libraries.

Includes the John Carter Brown Library.

Includes the General Library: Doe, Moffitt, Bancroft, Anthropology, Art History/Classics, Astronomy-Mathematics-Statistics, Bioscience and Natural Resources, Business \& Economics, Chemistry, East Asian (including Center for Chinese Studies), Earth Sciences, Education- Psychology, Engineering, Environmental Design, Music, Optometry, Physics, Public Health (including Health Sciences Information Services, and Occupational \& Environmental Health), and Social Welfare libraries and the Northern Regional Library Facility.

Excludes Affiliated Libraries: Architectural Slide, Continuing Education of the Bar, Earthquake Engineering, Ethnic Studies, Giannini, Institute of Governmental Studies, Institute of Industrial Relations, Institute of International Studies, Institute of Transportation Studies, and Water Resources Center libraries, Law Library, and various departmental libraries (French, History, Philosophy, Rhetoric, and Slavic Languages and Literature).

Beginning 2004-05, UCB salary figures include administrative stipends, where applicable. 
CALIFORNIA, DAVIS

\section{CALIFORNIA, LOS ANGELES}

\section{CALIFORNIA, RIVERSIDE}

CALIFORNIA, SAN DIEGO

CALIFORNIA, SANTA BARBARA CASE WESTERN RESERVE

CANADA INSTITUTE FOR SCIENTIFIC AND TECHNICAL INFORMATION
Includes the Peter J. Shields library, the Physical Sciences and Engineering library, the Carlson Health Sciences Library, and the Agricultural and Resource Economic Library on the Davis campus, as well as the Medical Center Library on the Sacramento campus.

Librarians reported as department heads have received administrative stipends that were not included prior to the 2004-05 survey.

Includes the Arts Library, College Library (Undergraduate Library), Eugene and Maxine Rosenfeld Management Library, Music Library, Richard C.

Rudolph East Asian Library, Science \& Engineering Library, Social Sciences and Humanities Library (Charles E. Young Research Library), and the Southern Regional Library Facility. Also includes data for 12 affiliated libraries on the UCLA campus including the American Indian Studies Center, African American Studies Center, Asian American Studies Center, Chicano Studies Research Center, Ethnomusicology Archive, Film \& Television Archive, Graduate School of Education \& Information Studies, Institute for Social Science Research, Latin American Center, Olive View Medical Center, Grant M. Hunt English Reading Room, and William Andrews Clark Memorial Library.

Data for the Hugh and Hazel Darling Law Library and the Louise M. Darling Biomedical Library are reported in ARL Law Library and ARL Medical Library surveys respectively. Data for Louise M. Darling Biomedical Library includes information for the Pacific Southwest Regional Medical Library, an NLM-funded program that is part of the National Network/Libraries of Medicine based in the Biomedical Library.

Librarians who are department heads have received administrative stipends since January 1998, however these stipends were not included in the salaries reported to ARL prior to 2003. Beginning with the 2003 survey, UCLA now includes those stipends in salaries reported for department heads.

The Law Library Director position is currently in recruitment. The Associate Law Librarian is serving in interim capacity.

The General Library Survey includes two Council of Library and Information Resources (CLIR) Fellows.

The beginning professional salary is scheduled to increase to $\$ 41,292$ on October 1, 2007.

Includes Rivera Library (serving the College of Humanities, Arts, and Social Sciences, the School of Education, and the Graduate School of Management), the Science Library (serving the College of Natural and Agricultural Sciences and the College of Engineering), and the Palm Desert Campus Library (serving the Graduate School of Management and the Graduate Division).

Excludes the Media and Music libraries, which have no librarian employees. Includes Special Collections, Social Sciences \& Humanities, Arts, Science and Engineering, and International Relations \& Pacific Studies libraries, as well as the Scripps Institution of Oceanography, and the Center for Library Instruction and Computing Services.

Includes the Main and Arts libraries.

Includes the Kelvin Smith (main) library, the Harris Library of the Mandel School of Applied Social Sciences, and the Kulas Music Library.

Includes all branch libraries. 


\section{CHICAGO}

CINCINNATI

COLORADO

COLORADO STATE

CONNECTICUT

CORNELL

DARTMOUTH

DUKE

EMORY

FLORIDA

FLORIDA STATE

GEORGETOWN

GEORGIA

GEORGIA TECH

GUELPH

HARVARD

HAWAII

HOUSTON
Includes all libraries: Science, Medicine, and Law.

Includes main campus only; branch campuses are not included.

Includes Norlin Library, Music, Business, Math/Physics, Engineering, and

Earth Sciences libraries.

Includes Main Campus Library, Atmospheric Sciences Library, and

Veterinary Medicine Library.

Includes Avery Point, Babbidge, Greater Hartford, Stamford, Torrington,

Waterbury, Archives and Special Collections, Art and Design, Maps, Music, and Pharmacy libraries.

Includes Africana, Engineering, Entomology, Fine Arts, Geneva Experiment Station, Hotel Administration, Management, Mann, Math, Music, ILR, Olin/ Kroch/Uris, Physical Sciences, and Veterinary Medicine libraries.

Includes Baker-Berry Library, Rauner Special Collections Library, Kresge Physical Sciences Library, Feldberg Business \& Engineering Library, Sherman Art Library, Paddock Music Library, Dartmouth College Records Center.

Includes Divinity, Perkins/Boston, Lilly, Music, Vesic, Biology and Environmental Sciences, Marine, and Rare Book, Manuscripts, and Special Collections libraries.

All salaries are effective September 1, 2007 and include the Main Library (which includes Chemistry, Music and Media, and the Manuscripts, Archives and Rare Books Library); Theology Library, and Oxford College Library. Includes Humanities and Social Science Library, Science Library, Music Library, Architecture and Fine Arts Library, Education Library, Journalism Library, and the Judaica Library.

Includes Strozier, Dirac (Science), Allen (Music), Goldstein, and Career Center libraries, as well as the Panama City ARC, the Ringling Museum in Sarasota, and the Heritage Protocol Grant.

Include Woodstock Theological Library and the National Reference Center for Bioethics Literature (Bioethics Library).

All libraries have different ranking structures.

Includes the Main Library, Student Learning Center, Science Library, Curriculum Materials Library, Tifton Agricultural Center Library, Griffin Agricultural Center Library.

Includes the Main and Architecture libraries.

Includes the Main Campus Libraries (McLaughlin Library, OVC Learning Commons), and the Guelph Humber Learning Commons.

Beginning professional salary unchanged from 2006-07 due to current and on-going labor negotiations.

Includes all libraries on the Boston and Cambridge campuses, including the Schlesinger Library at Radcliffe College.

Excludes Biblioteca Berenson (Florence, Italy), Dunbarton Oaks Research Libraries (Washington, DC), and the Center for Hellenic Studies (Washington, DC).

Includes the Hamilton and Sinclair libraries.

UH Library is reporting 24 additional positions for 2006-07. This is a result of a decision to include professional (non-librarians) for the first time.

Includes the M.D. Anderson Library, the Jenkins Architecture \& Art Library, the Music Library, the Pettey Optometry Library, and the Pharmacy Library. 
In 2005, a large number of positions were upgraded to "exempt" status. For the previous three years the UH Libraries had been reporting salaries for all exempt positions. However, the large reclassification had an impact on the data that does not reflect the salaries accurately, so starting in 2006-07 the UH libraries will report librarian salaries and staff salaries of positions that could conceivably be replaced by a librarian.

\section{HOWARD}

\section{ILLINOIS, CHICAGO ILLINOIS, URBANA INDIANA}

\section{IOWA}

IOWA STATE

\section{JOHNS HOPKINS}

\section{KANSAS}

\section{KENT STATE}

\section{KENTUCKY}

\section{LAVAL}

LIBRARY OF CONGRESS LOUISIANA STATE
Moorland Springarn Research Center is a separate library housed in the building of the Main Library and is included in the Main Library data. UIC is one campus, including Peoria, Rockford, and Urbana locations. Includes all main campus libraries. Includes all main campus libraries.

Excludes the Indianapolis School of Law, Dentistry, and Medicine Library; IUPUI University Library; Herron School of Art Library; Columbus Library; and Science and Engineering Library; as well as branch campuses at IU-East, IU-Kokomo, IU-Northwest, IU-Southeast, IU-South Bend, and IUPUI-Fort Wayne.

Includes Main Library and 10 branch libraries.

Includes Parks (main) Library and the Veterinary Medical branch library. Includes the Sheridan Libraries, the Friedheim Library, and the School of Advanced International Studies Library.

Salaries reported for the Main Library include the Lawrence and Edwards campuses. Salaries reported for the Medical Library include the Dykes Library.

Excludes the University of Kansas School of Medicine Library in Wichita, KS, and the Clendening History of Medicine Library in Kansas City, KS. Includes the Kent Campus (main) library, the Kent Campus branch libraries (Music, Architecture, Chemistry/Physics, Fashion, Map), and the regional campuses in Ashtabula, East Liverpool, Geauga, Salem, Stark, Trumbull, and Tuscarawas.

In previous years, faculty librarians were hired at the assistant professor level or above. In 2007, some faculty librarians were hired at the level of instructor, which is one step below assistant professor.

Includes the William T. Young Library (Main campus library), the Agricultural Information Center, Chemistry/Physics Library, Design Library, Education Library, Engineering Library, Equine Library, Fine Arts Library, Geological Sciences Library and Map Collections, Kentucky Transportation Center, Lexmark Information Center Mathematical Sciences Library, and Special Collections and Digital Programs.

Includes the law and medical libraries.

Salaries include Professional and Administrative positions. Includes Middleton Library and Hill Memorial Library.

While geographically in the same location (Baton Rouge), the Law School and School of Veterinary Medicine are considered separate campuses. Includes the Ekstrom (main) Library, the Art Library, the Music Library, and the University Archives. 


\section{McGILL}

\section{MCMASTER}

MANITOBA

\section{MARYLAND MASSACHUSETTS}

\section{MIAMI \\ MICHIGAN}

MISSOURI

MONTREAL

\section{NATIONAL AGRICULTURAL LIBRARY NEBRASKA}

\section{NEW MEXICO}

Includes Blackader (Art \& Architecture), Education, Howard Ross (Management), Schulich (Science \& Engineering), Marvin Duchow (Music), MacDonald campus (Agriculture), Islamic Studies, Humanities \& Social Sciences, Walter Hitsfeld (Geographic Information) libraries, Birks Reading Room, Digital Collections Program, Edward Rosenthall Mathematics and Statistics Library, Electronic Data Resource Service, Government Information Service, Rare Books and Special Collections Division, Library Technology Services, Collection Services, ILL/Document Delivery. Includes the Mills Memorial, Thode (Science \& Engineering), Innis (Business), and Health Sciences libraries.

Includes Elizabeth Dafoe, Albert D. Cohen, Sciences and Technology, Architecture/Fine Arts/Music, William R. Newman (Agriculture), D.S. Woods (Education), St. John's College, and Fr. Harold Drake libraries.

Excludes Neil John Maclean Health Sciences Library, Carolyn SiftonHelene Fuld St. Boniface Hospital, Victoria General Hospital, Seven Oaks General Hospital, Grace General Hospital, Concordia Hospital, Deer Lodge, Misercordia, and Riverview Health Center libraries.

Includes Main Library and all branch libraries.

Includes DuBois (main) library and the Integrated Sciences and Engineering Library.

Promotions and annual salary increases usually take effect in September of each year. All salaries will increase $2.5 \%$, and promotional increases of varying amounts will be implemented as soon as a new three-year collective bargaining contract is ratified.

Includes Main, Marine, Business, and Architecture libraries.

Includes Askwith Media, Asia, Biological Station, Dentistry, Fine Arts, Hatcher Graduate, Map, Art/Architecture/Engineering, Museums, Music, Science, UGL, Social Work, and Special Collections libraries.

Excludes Bentley, Clements, Kresge Business Administration, and Gerald R. Ford Presidential libraries.

Includes Main, Journalism, Engineering, Veterinary-Medical, and Archives libraries.

Includes Aménagement (Environmental Development), Bibliothéconomie et sciences de l'information (Library and Information Sciences), Botanique (Botany), Chimie (Chemistry), Didacthèque (Educational Resources), ÉPC-Biologie (Education-Communication-Psychology-PSychoeducationBiology), Géographie (Geography), Kinésiologie (Kinesiology), Lettres et sciences humaines (Humanities and Social Sciences), Livres rares et collections spéciales (Rare books and Special Collections), Mathématiques et informatique (Mathematics and Computer Sciences), Médecine vétérinaire (Veterinary), Musique (Music), Optométrie (Optometry), Physique (Physics), École polytechnique (Affiliated School), and HEC Montréal (Affiliated School) libraries.

Excludes Paramédicale (Paramedics), Droit (Law), and Santé (Health) Libraries.

All salaries are tracked with benefits.

Includes all University of Nebraska-Lincoln campus libraries: Love, Architecture, Engineering, Geology, Mathematics, Music, and C.Y. Thompson.

Includes Centennial Science and Engineering Library, Fine Arts Library, Parish Memorial Library, Zimmerman Library.

Excludes branch campuses in Gallup, Los Alamos, Taos, and Valencia. 


\section{NEW YORK}

\section{NORTH CAROLINA}

NORTH CAROLINA STATE

\section{NORTHWESTERN}

NOTRE DAME

OHIO

OHIO STATE

\section{OKLAHOMA STATE}

\section{OREGON}

\section{PENNSYLVANIA}

\section{PENNSYLVANIA STATE}

\section{PITTSBURGH}

\section{PRINCETON}

\section{PURDUE}

\section{QUEEN'S}

ROCHESTER
Includes Elmer Holmes Bobst, Institute of Fine Arts, Courant Institute of Mathematical Sciences, Real Estate, and Institute for Studies of the Ancient World libraries.

Includes main campus and branch libraries.

Excludes institute of marine science branch library.

Includes D. H. Hill (main), Design, Natural Resources, Textiles, Veterinary Medical, and College of Education Media Center.

Effective 2006-07, a number of librarians considered to be "assistant department heads" were reclassified from HDOTH (as reported in previous surveys) to the non-supervisory or specialist positions appropriate to their assignments. These positions do not spend the "bulk of their time in supervision" as per the HDOTH designation.

Includes the Science \& Engineering Library and the Schaffner Library. All salaries are as of September 1, 2007.

Includes the Main and branch campus libraries: Hesburgh, Chemistry/ Physics, Kellogg/Kroc Information Center, Fine Art/Architecture, Art Slide, Business Information Center, Life Sciences, Mathematics, and Engineering. Includes the Main campus only; all branch campuses excluded.

Includes the Main campus libraries, regional campus libraries, and libraries of the Agricultural Technical Institute and the Ohio Agricultural Research and Development Center.

Excludes several specialized departmental research libraries on the main campus which are not part of the University Libraries system.

Includes the Main Library, the Veterinary Medicine Library, the Architecture Library, Curriculum Materials, and branch campuses in Oklahoma City, Tulsa, and Okmulgee.

Includes Knight Library, Science Library, Math Library, and the Architecture and Allied Arts Library.

Includes Museum Library, Fine Arts Library, Physical Sciences Libraries, Business Library, Center for Advanced Judaic Studies Library, and Music Library.

Includes University Park (main) campus and branches at Abington, Altoona, Beaver, Berks, Delaware County, DuBois, Erie, Fayette, Harrisburg, Hazleton, Lehigh Valley, McKeesport, Mont Alto, New Kensington, Shenango, Schuylkill, Wilkes-Barre, Worthington Scranton, and York. Includes the University Library System and excludes branch campuses in Titusville, Johnstown, Bradford, and Greensburg.

Includes Main, Marquand, Mudd Library/Archives, Music, Architecture, Astrophysics, Chemistry, Engineering, Fine Hall (Sciences), Geosciences, Plasma Physics, Psychology, East Asian and Cotsen Children's Library. Includes the library system on the West Lafayette campus, consisting of 11 subject libraries, an undergraduate library, and an archives and special collections unit.

Excludes the libraries at regional campuses: North Central (Westville), Calumet (Hammond), and Indiana University - Purdue University, Fort Wayne.

Include Stauffer (Humanities \& Social Sciences), Douglas (Engineering \& Science), W.D. Jordan (Special Collections/Music).

Includes River Campus Libraries, Edward G. Miner Medical Library and Sibley Music Library. 


\section{RUTGERS}

\section{SASKATCHEWAN}

SOUTH CAROLINA

SOUTHERN CALIFORNIA

\author{
SUNY-ALBANY \\ SUNY-BUFFALO
}

\section{SUNY-STONY BROOK}

SYRACUSE

TEMPLE

TEXAS

TEXAS TECH

TORONTO

VANDERBILT
Includes New Brunswick Libraries (Alexander, Mabel Smith Douglass, and Kilmer libraries, as well as the Library of Science and Medicine and its branches), John Cotton Dana Library, Paul Robeson Library, and Technical \& Automated Services.

Excludes the School of Management and Labor Relations, and the Center for Alcohol Studies.

Includes Main, Natural Sciences, Veterinary Medicine, Education, and Engineering Libraries.

Number of employees reported is effective July 1, 2007.

Includes Thomas Cooper Library, South Caroliniana Library, Mathematics Library, Business Library, Music Library, and Film Library.

Includes Accounting, Applied Social Sciences, Architecture \& Fine Arts, Business, Science \& Engineering, Cinema-Television, Doheny (Research), East Asian, Gerontology, Grand (Depository), Leavey (Undergraduate), Music, Philosophy, and Special Collections.

Includes main campus and branch libraries.

Includes Arts \& Sciences libraries, Music Library, Special Collections (Archives, Poetry and Rare Books).

Excludes temporary hires, classified staff, employees at SL-2 level or discretionary increases.

Includes Main Campus, Math/Physics, Chemistry, Marine and Atmospheric Sciences libraries.

Includes Main campus Library, Science \& Technology Library, Geology Library, Math Library and Physics Library.

Excludes the College Reading Rooms.

Includes Paley (main) Library; Science Engineering and Architecture Library (SEAL); Blockson Afro-American Collection; Ambler Campus Library; Tyler School of Art Library.

In the Main Library, minimum beginning professional salary of $\$ 39,000$ is based on a 10-month contract, and minimum beginning salary for an 11 -month contract is $\$ 42,744$.

Includes the University of Texas Libraries, the Center for American History, and the Harry Ransom Humanities Research Center.

Some data for the Center for American History are not available.

All salaries are as of September 1, 2007, and include the University,

Southwest Collection/Special Collections, Architecture, and Vietnam Archives libraries.

Includes Robarts, Engineering \& Computer Science, Chemistry, Mathematics, School of Management, Physics, Faculty of Information Services, Media Commons, Astronomy, Rare Book, Criminology, Music, Earth Sciences, Dentistry, Pharmacy and East Asian libraries, as well as the Ontario Institute for Studies in Education, the University of Toronto branches at Scarborough and Mississauga, the University Archives, St. Michael's College, New College, and the Center for Industrial Relations, School of Management. Medical Library includes Gerstein Science and Information Center and Family and Community Medicine.

Includes the Central Library, Divinity Library, Peabody Library, Management Library, Science \& Engineering Library, Special Collections and University Archives, centralized Technical Services, Library Information Services, Administration, and TV News Archive. 


\section{VIRGINIA}

\section{WASHINGTON}

WASHINGTON STATE

WASINGTON U. - St. LOUIS

WATERLOO

WAYNE STATE

\section{WESTERN ONTARIO}

\section{WISCONSIN}

\section{YALE}

\section{YORK}

Includes Alderman (main), Clemons (undergraduate), Education, Fiske Kimball Fine Arts, Music, Charles L. Brown Science/Engineering system (6 libraries), Small Special Collections, and Darden Graduate Business libraries. Excludes the University of Virginia College at Wise.

Includes libraries on the Seattle, Bothell, and Tacoma campuses.

Salaries as of September 1, 2007.

Includes WSU Spokane, WSU Tri-Cities, WSU Vancouver, and WSU Energy Library.

Includes Central Library and departmental libraries in Art \& Architecture, Biology, Business, Chemistry, Earth Sciences, East Asian, Math, Music, Physics, and Social Work.

Includes Dana Porter, Davis Center, University Map, and Musagetes libraries. Includes the Purdy/Kresge Library, the Science and Engineering Library, the Adamany Undergraduate Library, and the Oakland Center Library Service Center.

The Wayne State law library includes the Neef Law Library. The Wayne State health sciences library includes the Shiffman Medical Library and the Pharmacy Learning Resources Center.

Includes the D.B. Weldon, Business, Education, Music, and Allyn \& Betty Taylor libraries.

Excludes Affiliated College Libraries: King's University College Library, Huron University College Library, Brescia University College Library, and St. Peter's Seminary Library.

Includes Memorial, College, Art, Biology, Business, Chemistry, Geography, Geology \& Geophysics, Mathematics, Music, Physics, Social Science Reference, Social Work, Steenbock Agricultural \& Life Sciences, and Wendt Engineering libraries.

Excludes the CIMC (School of Education), SLIS, Map, and Primate libraries. Includes Sterling Memorial Library, Cross Campus Library, Divinity Library, Mudd Library, Seeley G., Music Library, East Asia Library, Engineering Library, Arts Library, Government Documents, Beinecke Library, Social Science Libraries, Kline Science Library.

Includes Archives \& Special Collections, Leslie Frost Library, Peter F Bronfman Business Library, Map Library, Osgoode Hall Law Library, Scott Library, Sound \& Moving Image Library, and Steacie Science \& Engineering Library. 
APPENDICES 
APPENDIX A

ARL Member Libraries as of January 1, 2007

The Association of Research Libraries (ARL) represents the interests of 124 libraries that serve major North American research institutions. The ARL Statistics and Measurement program is organized around identifying, collecting, analyzing, and distributing quantifiable information describing the characteristics of research libraries.

Institution

\begin{tabular}{|c|}
\hline Alabama \\
\hline Alberta \\
\hline Arizona \\
\hline Arizona State \\
\hline Auburn \\
\hline Boston \\
\hline Boston College \\
\hline Brigham Young \\
\hline British Columbia \\
\hline Brown \\
\hline Berkeley, California \\
\hline California, Davis \\
\hline California, Irvine \\
\hline California, Los Angeles \\
\hline California, Riverside \\
\hline California, San Diego \\
\hline California, Santa Barbara \\
\hline Case Western Reserve \\
\hline Chicago \\
\hline Cincinnati \\
\hline Colorado \\
\hline Colorado State \\
\hline Columbia \\
\hline Connecticut \\
\hline Cornell \\
\hline Dartmouth \\
\hline Delaware \\
\hline Duke \\
\hline Emory \\
\hline Florida \\
\hline Flordia State \\
\hline George Washington \\
\hline Georgetown \\
\hline Georgia \\
\hline Georgia Tech \\
\hline Guelph \\
\hline Harvard \\
\hline Hawaii \\
\hline Houston \\
\hline Howard \\
\hline Illinois, Chicago \\
\hline Illinois, Urbana \\
\hline Indiana \\
\hline Iowa \\
\hline Iowa State \\
\hline
\end{tabular}

\section{Category Full Name of Institution}

University of Alabama

University of Alberta

University of Arizona

Arizona State University

Auburn University

Boston University

Boston College

Brigham Young University

University of British Columbia

Brown University

University of California, Berkeley

University of California, Davis

University of California, Irvine

University of California, Los Angeles

University of California, Riverside

University of California, San Diego

University of California, Santa Barbara

Case Western Reserve University

University of Chicago

University of Cincinnati

University of Colorado

Colorado State University

Columbia University

University of Connecticut

Cornell University

Dartmouth College

University of Delaware

Duke University

Emory University

University of Florida

Florida State University

George Washington University

Georgetown University

University of Georgia

Georgia Institute of Technology

University of Guelph

Harvard University

University of Hawaii

University of Houston

Howard University

University of Illinois at Chicago

University of Illinois at Urbana

Indiana University

University of Iowa

Iowa State University
Location

Tuscaloosa, Alabama

Edmonton, Alberta

Tucson, Arizona

Tempe, Arizona

Auburn, Alabama

Boston, Massachusetts

Boston, Massachusetts

Provo, Utah

Vancouver, British Columbia

Providence, Rhode Island

California, Berkeley

Davis, California

Irvine, California

Los Angeles, California

Riverside, California

La Jolla, California

Santa Barbara, California

Cleveland, Ohio

Chicago, Illinois

Cincinnati, Ohio

Boulder, Colorado

Fort Collins, Colorado

New York, New York

Storrs, Connecticut

Ithaca, New York

Hanover, New Hampshire

Newark, Delaware

Durham, North Carolina

Atlanta, Georgia

Gainesville, Florida

Tallahassee, Florida

Washington, D.C.

Washington, D.C.

Athens, Georgia

Atlanta, Georgia

Guelph, Ontario

Cambridge, Massachusetts

Honolulu, Hawaii

Houston, Texas

Washington, D.C.

Chicago, Illinois

Urbana, Illinois

Bloomington, Indiana

Iowa City, Iowa

Ames, Iowa 
Johns Hopkins

Kansas

Kent State

Kentucky

Laval

Louisiana State

Louisville

McGill

McMaster

Manitoba

Maryland

Massachusetts

MIT

Miami

Michigan

Michigan State

Minnesota

Missouri

Montreal

Nebraska

New Mexico

New York

North Carolina

North Carolina State

Northwestern

Notre Dame

Ohio

Ohio State

Oklahoma

Oklahoma State

Oregon

Pennsylvania

Pennsylvania State

Pittsburgh

Princeton

Purdue

Queen's

Rice

Rochester

Rutgers

Saskatchewan

South Carolina

Southern California

Southern Illinois

SUNY-Albany

SUNY-Buffalo

SUNY-Stony Brook

Syracuse

Temple

Tennessee

Texas

\section{P}

$\mathrm{S}$

$\mathrm{S}$

S

C

$\mathrm{S}$

S

C

C

C

S

$\mathrm{S}$

$\mathrm{P}$

$P$

$\mathrm{S}$

$\mathrm{S}$

S

S

C

S

S

$P$

$\mathrm{S}$

$\mathrm{S}$

$\mathrm{P}$
Johns Hopkins University

University of Kansas

Kent State University

University of Kentucky

Laval University

Louisiana State University

University of Louisville

McGill University

McMaster University

University of Manitoba

University of Maryland

University of Massachusetts

Massachusetts Institute of Technology

University of Miami

University of Michigan

Michigan State University

University of Minnesota

University of Missouri

University of Montreal

University of Nebraska-Lincoln

University of New Mexico

New York University

University of North Carolina

North Carolina State University

Northwestern University

University of Notre Dame

Ohio University

Ohio State University

University of Oklahoma

Oklahoma State University

University of Oregon

University of Pennsylvania

Pennsylvania State University

University of Pittsburgh

Princeton University

Purdue University

Queen's University

Rice University

University of Rochester

Rutgers University

University of Saskatchewan

University of South Carolina

University of Southern California

Southern Illinois University

University at Albany, State University of New York

University at Buffalo, State University of New York

State University of New York at Stony Brook

Syracuse University

Temple University

University of Tennessee

University of Texas
Baltimore, Maryland

Lawrence, Kansas

Kent, Ohio

Lexington, Kentucky

Quebec, Quebec

Baton Rouge, Louisiana

Louisville, Kentucky

Montreal, Quebec

Hamilton, Ontario

Winnipeg, Manitoba

College Park, Maryland

Amherst, Massachusetts

Cambridge, Massachusetts

Coral Gables, Florida

Ann Arbor, Michigan

East Lansing, Michigan

Minneapolis, Minnesota

Columbia, Missouri

Montreal, Quebec

Lincoln, Nebraska

Albuquerque, New Mexico

New York, New York

Chapel Hill, North Carolina

Raleigh, North Carolina

Evanston, Illinois

Notre Dame, Indiana

Athens, Ohio

Columbus, Ohio

Norman, Oklahoma

Stillwater, Oklahoma

Eugene, Oregon

Philadelphia, Pennsylvania

University Park, Pennsylvania

Pittsburgh, Pennsylvania

Princeton, New Jersey

West Lafayette, Indiana

Kingston, Ontario

Houston, Texas

Rochester, New York

New Brunswick, New Jersey

Saskatoon, Saskatchewan

Columbia, South Carolina

Los Angeles, California

Carbondale, Illinois

Albany, New York

Buffalo, New York

Stony Brook, New York

Syracuse, New York

Philadelphia, Pennsylvania

Knoxville, Tennessee

Austin, Texas 


\begin{tabular}{|c|c|}
\hline Texas A\&M & $S$ \\
\hline Texas Tech & $s$ \\
\hline Toronto & 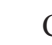 \\
\hline Tulane & $\mathrm{F}$ \\
\hline Utah & $S$ \\
\hline Vanderbilt & $\mathrm{F}$ \\
\hline Virginia & $s$ \\
\hline Virginia Tech & $S$ \\
\hline Washington & $S$ \\
\hline Washington State & $S$ \\
\hline Washington U.-St. Louis & $\mathrm{F}$ \\
\hline Waterloo & C \\
\hline Wayne State & $S$ \\
\hline Western Ontario & ( \\
\hline Wisconsin & $S$ \\
\hline Yale & $\mathrm{r}$ \\
\hline York & \\
\hline Boston Public Library & \\
\hline Canada Inst. SciTech Info. & \\
\hline Center for Research Libs. & \\
\hline Library of Congress & \\
\hline Natl. Agricultural Lib. & \\
\hline $\begin{array}{l}\text { Library \& Archives of } \\
\text { Canada }\end{array}$ & \\
\hline Natl. Library of Medicine & \\
\hline New York Public Library & \\
\hline New York State Library & \\
\hline Smithsonian Institution & \\
\hline
\end{tabular}

Texas A\&M University

Texas Tech University

University of Toronto

Tulane University

University of Utah

Vanderbilt University

University of Virginia

Virginia Polytechnic Institute \& State University

University of Washington

Washington State University

Washington University

University of Waterloo

Wayne State University

University of Western Ontario

University of Wisconsin

Yale University

York University

Boston Public Library

Canada Institute for Scientific \& Technical

Information

Center for Research Libraries

Library of Congress

National Agricultural Library

Library and Archives of Canada*

National Library of Medicine

New York Public Library

New York State Library

Smithsonian Institution
College Station, Texas

Lubbock, Texas

Toronto, Ontario

New Orleans, Louisiana

Salt Lake City, Utah

Nashville, Tennessee

Charlottesville, Virginia

Blacksburg, Virginia

Seattle, Washington

Pullman, Washington

St. Louis, Missouri

Waterloo, Ontario

Detroit, Michigan

London, Ontario

Madison, Wisconsin

New Haven, Connecticut

North York, Ontario

Boston, Massachusetts

Ottawa, Ontario

Chicago, Illinois

Washington, D.C.

Beltsville, Maryland

Ottawa, Ontario

Bethesda, Maryland New York, New York Albany, New York Washington, D.C. 


\section{APPENDIX B \\ TABLE NUMBERING CHANGES 1998-1999 TO 1999-2000}

From 1998-99 to 1999-2000 a number of new tables were added to ARL Annual Salary Survey publication and some old tables were renumbered. As a result of these changes, a new section was added, entitled U.S. ARL University Libraries, which includes Tables 26 and 29. Two new tables showing averages for all U.S. and Canadian institutions were also included as Table 3 and Table 4. The table below maps the old table-numbering scheme to the new one for purposes of comparison.

Old New

\section{SALARY LEVELS FOR STAFF IN ARL LIBRARIES}

$\begin{array}{rll}1 & 1 & \text { Distribution by Salary Level } \\ 12 & 2 & \text { Salary Trends in ARL University Libraries } \\ \text { N/A } & 3 & \text { Salary Trends in U.S. ARL University Libraries } \\ \text { N/A } & 4 & \text { Salary Trends in Canadian ARL University Libraries }\end{array}$

\section{ARL NONUNIVERSITY LIBRARIES}

5 Median and Beginning Professional Salaries in ARL Nonuniversity Libraries

6 Salary Trends in ARL Nonuniversity Libraries

\section{ARL UNIVERSITY LIBRARIES}

Filled Positions; Average, Median, Beginning Professional Salaries, And Average Years of Professional Experience in ARL University Libraries, FY 1999-2000

Beginning Professional Salaries in ARL University Libraries; Rank Order Table, FY 1998-99

Beginning Professional Salaries in ARL University Libraries; Rank Order Table, FY 1999-2000

Median Professional Salaries in ARL University Libraries; Rank Order Table, FY 1998-99

Median Professional Salaries in ARL University Libraries; Rank Order Table, FY 1999-2000

Average Professional Salaries in ARL University Libraries; Rank Order Table, FY 1998-99

Average Professional Salaries in ARL University Libraries; Rank Order Table, FY 1999-2000

Average, Median, and Beginning Professional Salaries
Summary of Rankings, FYs 1996-97 to 1999-2000

Distribution of Professional Staff in ARL University Libraries by Salary and Position, FY 1999-2000

Distribution of Professional Staff in ARL University Libraries by Salary, Sex, and Position, FY 1999-2000

Number and Average Salaries of ARL University Librarians by Position and Sex, FY 1999-2000

Number and Average Years of Experience of ARL University Librarians by Position and Sex, FY 1999-2000

Number and Average Salaries of ARL University Librarians by Years of Experience and Sex, FY 1999-2000 

by Position and Type of Institution, FY 1999-2000 by Position and Geographic Region, FY 1999-2000

\section{U.S. ARL UNIVERSITY LIBRARIES}

Average Salaries of U.S. ARL University Librarians by Position and Years of Experience, FY 1999-2000

Number and Average Salaries of Mino
by Position and Sex, FY 1999-2000

Number and Average Years of Experience of Minority U.S. ARL University Librarians by Position and Sex, FY 1999-2000

Number and Average Salaries of U.S. ARL University Librarians by Years of Experience and Sex, FY 1999-2000

Number and Average Salaries of Minority U.S. ARL University Librarians by Years of Experience and Sex, FY 1999-2000

\section{CANADIAN ARL UNIVERSITY LIBRARIES}

31 Filled Positions; Average, Median, and Beginning Professional Salaries; and Average Years of Professional Experience in Canadian ARL University Libraries, FY 1999-2000

32 Number and Average Salaries of Canadian ARL University Librarians by Position and Sex, FY 1999-2000

Number and Average Years of Experience of Canadian ARL University Librarians by Position and Sex, FY 1999-2000

34 Number and Average Salaries of Canadian ARL University Librarians by Years of Experience and Sex, FY 1999-2000

\section{ARL UNIVERSITY MEDICAL LIBRARIES}

Filled Positions; Average, Median, Beginning Professional Salaries; and Average Years of Professional Experience in ARL University Medical Libraries, FY 1999-2000

36 Beginning Professional Salaries in ARL University Medical Libraries; Rank Order Table, FY 1999-2000

Median Professional Salaries in ARL University Medical Libraries; Rank Order Table, FY 1999-2000

Average Professional Salaries in ARL University Medical Libraries; Rank Order Table, FY 1999-2000 by Position and Sex, FY 1999-2000 

by Position and Sex, FY 1999-2000

Number and Average Salaries of ARL University Medical Librarians by Years of Experience and Sex, FY 1999-2000

\section{ARL UNIVERSITY LAW LIBRARIES}

Filled Positions; Average, Median, Beginning Professional Salaries; and Average Years of Experience in ARL University Law Libraries, FY 1999-2000

Beginning Professional Salaries in ARL University Law Libraries; Rank Order Table, FY 1999-2000

Median Professional Salaries in ARL University Law Libraries; Rank Order Table, FY 1999-2000

Average Professional Salaries in ARL University Law Libraries; Rank Order Table, FY 1999-2000

Number and Average Salaries of ARL University Law Librarians by Position and Sex, FY 1999-2000

Number and Average Years of Expe
by Position and Sex, FY 1999-2000

Number and Average Salaries of ARL University Law Librarians by Years of Experience and Sex, FY 1999-2000 


\section{ARL Statistics and Measurement Program \\ Publications and Services \\ http://www.arl.org/stats/}

\section{ARL Academic Law and Medical Library Statistics}

ISSN 1088-1093 • Back issues are available • Standing order offered

Yearly report of data for collections' size and growth, materials and operating expenditures, staffing, and public service activities in ARL university law and medical libraries. Includes comparisons to the larger research collections at each university.

\section{ARL Preservation Statistics}

ISSN 1050-7442 • Back issues are available $\bullet$ Standing order offered

Annual compilation of data on current levels of preservation efforts and reports on the key organizational, functional, and fiscal components comprising ARL preservation programs.

\section{ARL Statistics}

ISSN 0147-2135 • Back issues are available • Standing order offered

Annual compilation of data on collection size and growth, materials and operating expenditures, staffing, and library services. The most comprehensive resource for information on research library operations and trends.

\section{$\operatorname{LibQUAL}+{ }^{\circledR}$}

LibQUAL $+{ }^{\circledR}$ is a suite of services that libraries use to solicit, track, understand, and act upon users' opinions of service quality. These services are offered to the library community by the Association of Research Libraries (ARL). The program's centerpiece is a rigorously tested Webbased survey bundled with training that helps libraries assess and improve library services, change organizational culture, and market the library. LibQUAL $+{ }^{\circledR}$ is on the Web at http://www.libqual.org/.

\section{User Surveys in Academic Libraries}

This workshop presents the basic concepts and steps for conducting a user survey: defining objectives, sampling, measurement scales, logistics, data analysis, and report writing.

\section{Web Development with XML: Design and Applications}

This workshop is designed for librarians, information professionals, and educators. It provides hands-on experience in developing interfaces for publishing on the Web.

\section{Customized Services}

For any dataset produced by ARL, libraries can request comparative institutional data and ARL will perform the analysis and provide tables and reports in the format best suited to the need.

For more information on any of these products or services, please contact stats@arl.org or call 202-296-2296. 